TRANSACTIONS OF THE

AMERICAN MATHEMATICAL SOCIETY

Volume 361, Number 12, December 2009, Pages 6283-6348

S 0002-9947(09)04936-8

Article electronically published on July 22, 2009

\title{
CONSTRUCTION OF CANONICAL COORDINATES FOR EXPONENTIAL LIE GROUPS
}

\author{
DIDIER ARNAL, BRADLEY CURREY, AND BECHIR DALI
}

\begin{abstract}
Given an exponential Lie group $G$, we show that the constructions of B. Currey, 1992, go through for a less restrictive choice of the Jordan-Hölder basis. Thus we obtain a stratification of $\mathfrak{g}^{*}$ into $G$-invariant algebraic subsets, and for each such subset $\Omega$, an explicit cross-section $\Sigma \subset \Omega$ for coadjoint orbits in $\Omega$, so that each pair $(\Omega, \Sigma)$ behaves predictably under the associated restriction maps on $\mathfrak{g}^{*}$. The cross-section mapping $\sigma: \Omega \rightarrow \Sigma$ is explicitly shown to be real analytic. The associated Vergne polarizations are not necessarily real even in the nilpotent case, and vary rationally with $\ell \in \Omega$. For each $\Omega$, algebras $\mathcal{E}^{0}(\Omega)$ and $\mathcal{E}^{1}(\Omega)$ of polarized and quantizable functions, respectively, are defined in a natural and intrinsic way.

Now let $2 d>0$ be the dimension of coadjoint orbits in $\Omega$. An explicit algorithm is given for the construction of complex-valued real analytic functions $\left\{q_{1}, q_{2}, \ldots, q_{d}\right\}$ and $\left\{p_{1}, p_{2}, \ldots, p_{d}\right\}$ such that on each coadjoint orbit $\mathcal{O}$ in $\Omega$, the canonical 2 -form is given by $\sum d p_{k} \wedge d q_{k}$. The functions $\left\{q_{1}, q_{2}, \ldots, q_{d}\right\}$ belong to $\mathcal{E}^{0}(\Omega)$, and the functions $\left\{p_{1}, p_{2}, \ldots, p_{d}\right\}$ belong to $\mathcal{E}^{1}(\Omega)$. The associated geometric polarization on each orbit $\mathcal{O}$ coincides with the complex Vergne polarization, and a global Darboux chart on $\mathcal{O}$ is obtained in a simple way from the coordinate functions $\left(p_{1}, \ldots, p_{d}, q_{1}, \ldots, q_{d}\right)$ (restricted to $\mathcal{O}$ ). Finally, the linear evaluation functions $\ell \mapsto \ell(X)$ are shown to be quantizable as well.
\end{abstract}

\section{INTRODUCTION}

Let $G$ be a real solvable Lie group with Lie algebra $\mathfrak{g}$ for which the exponential mapping exp : $\mathfrak{g} \rightarrow G$ is a diffeomorphism. It is shown in [6] that each non-trivial coadjoint orbit $\mathcal{O}$ in $\mathfrak{g}^{*}$ admits a global Darboux chart: there is a system of global coordinates $c=\left(p_{1}, p_{2}, \ldots, p_{d}, q_{1}, q_{2}, \ldots, q_{d}\right)$ for $\mathcal{O}$ for which $c^{*}\left(\sum_{k} d p_{k} \wedge d q_{k}\right)$ is the canonical Kostant-Souriau 2 -form on $\mathcal{O}$. We shall call any such system of coordinates "canonical coordinates" for $\mathcal{O}$. Using a different method, systems of canonical coordinates are built in [2] for families of orbits simultaneously using a "good" flag $\left(\mathfrak{g}_{j}\right)$ of real subalgebras of $\mathfrak{g}$. It is then shown in [2] that this construction has important applications to star products and to Plancherel theory for exponential solvable Lie groups.

Received by the editors July 2, 2007.

2000 Mathematics Subject Classification. Primary 22E25, 22E27; Secondary 53D50.

The first author would like to thank the CNRS for its support and the Faculté de Sciences de Bizerte for its hospitality.

The second author would like to thank the Université de Bourgogne for their hospitality and support.

The third author would like to thank the Université de Bourgogne for its hospitality during his stay. He would also like to thank the DGRSRT of Tunisia for its support.

(C)2009 American Mathematical Society 
In this paper we are concerned only with the construction and properties of canonical coordinates. The results herein are the outcome of an attempt to understand how the construction of [2] might be combined with the constructions of [5], where explicit orbital parametrizations are also defined on large classes of orbits simultaneously. The advantage of the methods of [5] is that the constructions are extremely explicit and canonical, once a suitable basis for the complexification of the Lie algebra is chosen. We will show that a combination of the two approaches obtains a construction of systems of canonical coordinates that are explicitly associated with the stratification and orbital parameters of [5], that are canonical once a suitable basis is chosen, and that exhibit better behavior.

In the construction of [2, complications arise from the following issues. One considers the set $V_{\left(d_{1}, \ldots, d_{n}\right)}$ of $\ell \in \mathfrak{g}^{*}$ for which the dimension of the $G_{j}$-orbit $\left(G_{j}=\exp \left(\mathfrak{g}_{j}\right)\right)$ of $\left.\ell\right|_{\mathfrak{g}_{j}}$ is exactly $2 d_{j}, 1 \leq j \leq n$. Since not all of the subalgebras $\mathfrak{g}_{j}$ are ideals, $V_{\left(d_{1}, \ldots, d_{n}\right)}$ is not $G$-invariant. Moreover, a Borel cross-section $\Sigma^{(j)}$ for the action of $G_{j}$ on $\mathfrak{g}_{j}^{*}$ is required for each $j$ such that elements of $\Sigma^{(j+1)}$ restrict to elements of $\Sigma^{(j)}$. The construction depends upon existence of these objects, and the proof of their existence depends upon a large number of choices. Secondly, as is described in [3], the coordinate functions that result from the construction in [2] are not necessarily well-behaved algebraically. For example, the real geometric polarization associated with the canonical coordinates may not correspond to any real algebraic polarization in $\mathfrak{g}$. In Section 1 we describe these issues in some detail and give three examples to illustrate them.

In Section 2 we describe the fine stratification procedure of [5] and its relationship with complex Vergne polarizations. Given a suitably chosen basis for the complexification of $\mathfrak{g}$, and the corresponding flag of complex ideals, the constructions are explicit and canonical. The conditions we require of this basis are slightly weaker than the conditions set forth in 5, and therefore the results of Sections 2, 3, and 4 represent slight but significant generalizations of some of the results contained in [5]. The significance of this paper is that these constructions apply automatically to those ideals in the chosen flag which have real forms.

The fine stratification of $\mathfrak{g}^{*}$ consists of disjoint, algebraically-defined, $G$-invariant subsets $\Omega=\Omega_{\mathbf{e}, \mathbf{j}}$ of $\mathfrak{g}^{*}$ which we call fine layers. All orbits in a given fine layer have the same dimension, and the map from linear functionals $\ell \in \Omega$ to their complex Vergne polarizations is rational and non-singular. Working in a given fine layer $\Omega$ whose orbits have dimension $2 d>0$, rational functions $U_{k}, V_{k}: \Omega \rightarrow \mathfrak{s}, 1 \leqslant k \leqslant d$, are built. The vectors $U_{k}(\ell), V_{j}(\ell)$, are orthogonal for the Kostant-Souriau 2form, and for each $\ell \in \Omega$, the complex tangent space to the coadjoint orbit of $\ell$ is generated by the infinitesimal action $\xi^{U_{k}(\ell)}, \xi^{V_{k}(\ell)}$ of these vectors. In Section 3 we describe this construction and compute the Kostant bi-vector on $\mathfrak{g}^{*}$ explicitly as a combination of $\xi^{U_{k}(\ell)} \wedge \xi^{V_{k}(\ell)}$.

In order to describe the coadjoint orbit space, one must identify those coordinates where $G$ "acts by dilation", and this results in a refinement of the fine stratification which we call the ultrafine stratification. For each ultrafine layer $\Omega$, an explicit cross-section $\Sigma$ was built in [5]. We recall this construction (for our slightly less restrictive choice of basis), and we give a new, simple presentation of $\Sigma$ in Section 4 that clarifies the connection between $\Sigma$ and the constructions of the objects $U_{k}, V_{k}$. Moreover, we show that the corresponding cross-section map is real analytic on each ultrafine layer. As indicated above, these constructions also apply to each 
of the normal subgroups $G_{m}$ belonging to the chosen flag. As a result, crosssections $\Sigma^{m}$ for $G_{m}$-orbits in $\mathfrak{g}_{m}^{*}$ are obtained for all the ideals $\mathfrak{g}_{m}$ canonically, by a single procedure. Our new presentation of cross-sections permits us in Section 5 to describe the relationship between layers and cross-sections in $\mathfrak{g}^{*}$ and $\mathfrak{g}_{m}^{*}$.

All this then sets the stage for a canonical construction, beginning in Section 6] and concluded in Section 7 of complex-valued functions $p_{1}, \ldots, p_{d}, q_{1}, \ldots, q_{d}$ on the ultrafine layer $\Omega$, each of which is real analytic in a neighborhood of $\Omega$. For the normal subgroup $G_{m}$ belonging to the chosen flag of codimension one or two, the machinery developed in earlier sections reduces this construction to an induction step from $G_{m}$ to $G$. In Section 6 this induction step is begun, and when the orbital dimension increases from $G_{m}$ to $G$, the new coordinate functions are explicitly described in terms of elementary functions.

In Section 7 we describe an algebra of polarized functions on $\Omega$ and we complete the construction, obtaining the system of complex-valued analytic functions $p_{1}, \ldots, p_{d}, q_{1}, \ldots, q_{d}$ on $\Omega$. We show that the functions $q_{r}$ are polarized functions on $\Omega$, and that the two-form

$$
\sum_{r=1}^{d} d p_{r} \wedge d q_{r}
$$

restricts to the canonical two-form on each orbit in $\mathcal{O} \subset \Omega$. If the Vergne polarizations in $\Omega$ have real forms, then for each orbit in $\Omega$ the coordinates can be twisted (again in an analytic way) so as to be real, thus yielding a global Darboux chart. Otherwise for some $r$ both $p_{r}$ and $q_{r}$ map the orbit onto the complex plane, and a global Darboux chart is obtained by taking real and imaginary parts in these directions. In either case, because the functions $q_{r}$ are polarized, the geometric polarization of each orbit associated with the system of functions above coincides with the Vergne polarization. Moreover the functions $p_{r}$, as well as the coordinate functions, are quantizable.

Finally, we remark that the relationship between the preceding constructions and the results of [2] is simplified if one can arrange things so that $\mathfrak{s}_{j}=\left(\mathfrak{g}_{j}\right)_{\mathbb{C}}$ for all $j$, that is, if $G$ is completely solvable. In [1, it is shown that if this is the case, then the method of construction for canonical coordinates used in 2 adapts readily to the explicit stratification and cross-sections described above. On the other hand, there are contexts in which it is desirable not to use a real Jordan-Hölder basis, and in this paper we do not require that a real basis be used, even in the nilpotent case. Thus our constructions apply to a broad class of complex polarizations. This increased flexibility comes with a price: when some of the ideals $\mathfrak{s}_{j}$ do not have real forms, then the adaptation of the method of [2] is less straightforward. In this case the induction step from $G_{m}$ to $G$, particularly as it occurs in Section 6 involves a variety of cases.

\section{REAL FLAG AND CANONICAL COORDINATES}

We begin by setting some notation which will be used throughout the paper. Let $\mathfrak{g}$ be a real Lie algebra and $G$ the corresponding connected and simply connected Lie group. We assume that the group $G$ is exponential: the exponential mapping $\exp : \mathfrak{g} \rightarrow G$ is a global diffeomorphism. We recall that this is equivalent to the condition that $\mathfrak{g}$ is solvable and that any root of $\mathfrak{g}$ has the form $(1+i \alpha) c$ with $c \in \mathfrak{g}^{*}$ and $\alpha \in \mathbb{R}$. We denote the complexification $\mathfrak{g}_{\mathbb{C}}$ of $\mathfrak{g}$ by $\mathfrak{s}$. For $Z \in \mathfrak{s}$, denote the 
real part of $Z$ by $\mathfrak{R e} Z$, and the imaginary part of $Z$ by $\mathfrak{I m} Z$. (We also use these symbols to denote real and imaginary parts of a complex number.) We regard the real dual $\mathfrak{g}^{*}$ as a real subspace of the complex vector space $\mathfrak{s}^{*}$ in the natural way. Given $s \in G, Z \in \mathfrak{s}$, and $\ell \in \mathfrak{g}^{*}$, we denote both the adjoint and coadjoint actions multiplicatively: $A d(s) Z=s Z$ and $A d^{*}(s) \ell=s \ell$. Note that the adjoint (resp. coadjoint) action extends to $\mathfrak{s}$ (resp. $\mathfrak{s}^{*}$ ) as well. Denote the coadjoint orbit of $\ell$ by $\mathcal{O}_{\ell}$. Given $\ell \in \mathfrak{g}^{*}$, we use the notation $\ell(Z)$ for the evaluation of $\ell$ at $Z \in \mathfrak{s}$ and we shall denote $\ell([Y, Z])$ simply by $\ell[Y, Z]$ for $Y, Z \in \mathfrak{s}$.

Let $\mathfrak{t}$ be any subset of $\mathfrak{s}$. We say that $\mathfrak{t}$ is real if $\overline{\mathfrak{t}}=\mathfrak{t}$. For any $\ell \in \mathfrak{g}^{*}$, and any subset $\mathfrak{t}$ of $\mathfrak{s}$, we set

$$
\mathfrak{t}^{\ell}=\{Z \in \mathfrak{s} \mid \ell[Z, X]=0, \text { for all } X \in \mathfrak{t}\} .
$$

If $\mathfrak{t}$ is a subalgebra of $\mathfrak{s}$ we put $\mathfrak{t}(\ell)=\mathfrak{t}^{\ell} \cap \mathfrak{t}$. Recall that if $\mathfrak{t}$ is a subalgebra of $\mathfrak{g}$, then $\mathfrak{t}(\ell)$ is the Lie algebra of the stabilizer of $\left.\ell\right|_{\mathfrak{t}}$ under the coadjoint action of $T=\exp \mathfrak{t}$. If $\mathfrak{t}$ is an ideal in $\mathfrak{s}$, then $\mathfrak{t}$ and $\mathfrak{t}^{*}$ are invariant subspaces for the adjoint and coadjoint actions of $G$ respectively, and we denote the restricted actions multiplicatively as well. In this case the restriction map $\pi_{\mathfrak{t}}: \mathfrak{g}^{*} \rightarrow \mathfrak{t}^{*}$ intertwines the actions of $G$ on $\mathfrak{g}^{*}$ and $\mathfrak{t}^{*}$, and we denote its kernel by $\operatorname{ker}\left(\pi_{\mathfrak{t}}\right)=\mathfrak{t}^{\perp}$. Thus we shall identify $\mathfrak{t}^{*}$ with $\mathfrak{g}^{*} / \mathfrak{t}^{\perp}$.

Fix $\ell \in \mathfrak{g}^{*}$; we identify the tangent space $T_{\ell}\left(\mathfrak{g}^{*}\right)$ with $\mathfrak{g}^{*}$ in the canonical way. Complexifications are naturally identified: $T_{\ell}\left(\mathfrak{g}^{*}\right)_{\mathbb{C}}$ is identified with $\mathfrak{s}^{*}$, and the complex dual space of $T_{\ell}\left(\mathfrak{g}^{*}\right)_{\mathbb{C}}$ is identified with $\mathfrak{s}$. For $X \in \mathfrak{g}$, let $\xi_{\ell}^{X}$ denote the tangent vector at $\ell$ defined by $\ell[X, \cdot]$; we also have

$$
\xi_{\ell}^{X} \psi=\left.\frac{d}{d t}\right|_{t=0} \psi(\exp (-t X) \ell)
$$

where $\psi$ is any smooth function defined in a neighborhood of $\ell$. Let $\mathcal{O}$ be the coadjoint orbit through $\ell$ and note that $T_{\ell}(\mathcal{O})=\xi_{\ell}^{\mathfrak{g}}=\{\ell[X, \cdot] \mid X \in \mathfrak{g}\}$. Of course $\xi_{\ell}$ extends to the complexification $\mathfrak{s}$ and the image of $\mathfrak{s}$ under $\xi_{\ell}$ is the complexification $T_{\ell}(\mathcal{O})_{\mathbb{C}}$ of $T_{\ell}(\mathcal{O})$. For each $X \in \mathfrak{s}$ denote the resulting vector field on $\mathfrak{g}^{*}$ by $\xi^{X}$; recall that $X \mapsto \xi^{X}$ is a Lie algebra homomorphism.

Let $\omega_{\mathcal{O}}$ be the canonical 2 -form (the Kostant-Souriau form) on the coadjoint orbit $\mathcal{O}$. In [6] it is shown that there exists a globally-defined system of canonical coordinates, that is a global Darboux chart,

$$
c=\left(p_{1}, p_{2}, \ldots, p_{d}, q_{1}, q_{2}, \ldots, q_{d}\right)
$$

on $\mathcal{O}$. In [2], using a quite different method, the authors define a partition of $\mathfrak{g}^{*}$ into finitely many $G$-invariant Borel sets and give an induction argument for the existence of a parametrization of each Borel set, which includes global Darboux charts defined simultaneously for all orbits in each member of the partition. It is these global Darboux charts to which we now direct our attention.

Let $\left(\mathfrak{g}_{j}\right)$ be a flag of subalgebras of $\mathfrak{g}$ so that $(0)=\mathfrak{g}_{0} \subset \mathfrak{g}_{1} \subset \cdots \subset \mathfrak{g}_{n}=\mathfrak{g}$ and $\operatorname{dim}\left(\mathfrak{g}_{j}\right)=j, 1 \leq j \leq n$. Then, for each $j, \mathfrak{g}_{j-1}$ is an ideal in $\mathfrak{g}_{j}$. Assume that if $\mathfrak{g}_{j}$ is not an ideal in $\mathfrak{g}$, then both $\mathfrak{g}_{j-1}$ and $\mathfrak{g}_{j+1}$ are as well. Choose an ordered basis $\left(X_{1}, X_{2}, \ldots, X_{n}\right)$ for $\mathfrak{g}$ with $X_{j} \in \mathfrak{g}_{j}, 1 \leq j \leq n$. The proof of [2, 1.6 Théorème] is presented inductively and is based upon the following idea.

For simplicity of notation, put $\pi=\pi_{\mathfrak{g}_{n-1}}$. Let $\mathcal{O}$ be any coadjoint orbit in $\mathfrak{g}^{*}$, and let $\operatorname{dim}(\mathcal{O})=2 d$ with $d>0$. Fix a base point $g \in \mathcal{O}$ with $f=\pi(g)$ and put $\mathcal{O}_{f}=G_{n-1} f \subset \mathfrak{g}_{n-1}^{*}$. Suppose that we are given canonical coordinates 
$p_{1}^{\circ}, p_{2}^{\circ}, \ldots, p_{d^{\circ}}^{\circ}, q_{1}^{\circ}, q_{2}^{\circ}, \ldots, q_{d^{\circ}}^{\circ}$ for $\mathcal{O}_{f}$. If $\mathfrak{g}_{n-1}+\mathfrak{g}^{\ell}=\mathfrak{g}$, then $d^{\circ}=d$ and $\mathcal{O}_{f}$ is symplectomorphic with $\mathcal{O}$ via the map $\pi$. In this case the given coordinates for $\mathcal{O}_{f}$ lift to give canonical coordinates for $\mathcal{O}$. On the other hand, if $\mathfrak{g}_{n-1}+\mathfrak{g}^{\ell} \neq \mathfrak{g}$, then $d^{\circ}=d-1$, and for each $\ell \in \mathcal{O}$ there is a unique real number $q(\ell)$ such that

$$
\pi\left(\exp \left(q(\ell) X_{n}\right) \ell\right) \in \mathcal{O}_{f}
$$

We define $q_{d}=q$. Setting

$$
q_{r}(\ell)=q_{r}^{\circ}\left(\pi\left(\exp \left(q(\ell) X_{n}\right) \ell\right)\right), p_{r}(\ell)=p_{r}^{\circ}\left(\pi\left(\exp \left(q(\ell) X_{n}\right) \ell\right)\right)
$$

for $1 \leq r \leq d-1$, and $p_{d}(\ell)=\ell\left(X_{n}\right)$, one easily finds that $p_{1}, p_{2}, \ldots, p_{d}, q_{1}, q_{2}, \ldots, q_{d}$ are canonical coordinates for $\mathcal{O}$.

Hence one has a recursive construction of canonical coordinates for $\mathcal{O}$ : setting $\mathcal{O}_{\pi_{j}(g)}=G_{j} \pi_{j}(g)$, execution of the preceding induction stepping first from $\mathcal{O}_{\pi_{1}(g)}$ to $\mathcal{O}_{\pi_{2}(g)}$, then from $\mathcal{O}_{\pi_{2}(g)}$ to $\mathcal{O}_{\pi_{3}(g)}$, and so on, yields canonical coordinates $p_{k}^{(j)}, q_{k}^{(j)}$, $k=1,2, \ldots, d_{j}$, for $\mathcal{O}_{\pi_{j}(g)}, 1 \leq j \leq n$. Note that the preceding depends upon the initial choice of the base point $g \in \mathcal{O}$.

Now in [2, 1.6 Théorème] this idea is used to obtain canonical coordinates simultaneously for families of orbits. Base points are just members of Borel cross-sections for $G$-invariant Borel subsets of $\mathfrak{g}^{*}$ in which all orbits have the same dimension. The construction requires that such objects be specified in each subalgebra $\mathfrak{g}_{j}$ and that the cross-sections be "nested", as follows.

Fix $1 \leq j<n$ and suppose we have a family of cross-sections $\Sigma^{(t)}$ for the action of $G_{t}$ on $\mathfrak{g}_{t}^{*}, 1 \leq t \leq j$, such that $\pi_{t}\left(\Sigma^{(t+1)}\right) \subset \Sigma^{(t)}$ for $t<j$. Let $\sigma^{(j)}: \mathfrak{g}_{j}^{*} \rightarrow \Sigma^{(j)}$ be the cross-section map, sending $\ell$ to the unique point in $G_{j} \ell \cap \Sigma^{(j)}$. Write $\mathfrak{g}_{j}^{*}$ as the disjoint union of the sets $V_{\mathbf{d}}^{(j)}=V_{\left(d_{1}, \ldots, d_{j}\right)}^{(j)}$, where

$$
V_{\mathbf{d}}^{(j)}=\left\{\ell \in \mathfrak{g}_{j}^{*} \mid \operatorname{dim} G_{t}\left(\left.\ell\right|_{\mathfrak{g}_{t}}\right)=2 d_{t}, 1 \leq t \leq j\right\}
$$

Then in $\mathfrak{g}_{j+1}^{*}$,

$$
\left(\pi_{j}\right)^{-1}\left(V_{\mathbf{d}}^{(j)}\right)=V_{\left(d_{1}, \ldots, d_{j}, d_{j}\right)}^{(j+1)} \cup V_{\left(d_{1}, \ldots, d_{j}, d_{j}+1\right)}^{(j+1)} .
$$

Complications arise from the fact that $\mathfrak{g}_{t}$ is not necessarily an ideal in $\mathfrak{g}_{j}$ for $t<j-1$, and hence the space $V_{\mathbf{d}}^{(j)}$ is not necessarily $G_{j}$-invariant.

Define $\Sigma_{\mathbf{d}}^{(j)}=\Sigma^{(j)} \cap V_{\mathbf{d}}^{(j)}$. Choose a cross-section $\tilde{\Sigma}^{(j)}$ for the $G_{j+1}$-action on $\mathfrak{g}_{j}^{*}$; then $\sigma^{(j)}\left(\tilde{\Sigma}^{(j)}\right)$ is still a cross-section for this action and $\sigma^{(j)}\left(\tilde{\Sigma}^{(j)}\right) \subset \Sigma^{(j)}$. We decompose it as

$$
\sigma^{(j)}\left(\tilde{\Sigma}^{(j)}\right)=\bigcup_{\mathbf{d}}\left(\sigma^{(j)}\left(\tilde{\Sigma}^{(j)}\right) \cap \Sigma_{\mathbf{d}}^{(j)}\right)=\bigcup_{\mathbf{d}} \tilde{\Sigma}_{\mathbf{d}}^{(j)}
$$

But $\tilde{\Sigma}_{\mathbf{d}}^{(j)}=\tilde{\Sigma}_{\left(d_{1}, \ldots, d_{j}\right)}^{(j)}$ contains points in $\pi_{j}\left(V_{\mathbf{d}^{\prime}}^{(j+1)}\right)$ with the two possibilities $\mathbf{d}^{\prime}=$ $\left(d_{1}, \ldots, d_{j}, d_{j}\right)$ and $\mathbf{d}^{\prime}=\left(d_{1}, \ldots, d_{j}, d_{j}+1\right)$. Thus we finally define the cross-section $\Sigma^{(j+1)}$ by $\Sigma^{(j+1)}=\bigcup_{\mathbf{d}^{\prime}} \Sigma_{\mathbf{d}^{\prime}}^{(j+1)}$, where

$$
\Sigma_{\left(d_{1}, \ldots, d_{j}, d_{j}+1\right)}^{(j+1)}=\left\{\ell \in V_{\left(d_{1}, \ldots, d_{j}, d_{j}+1\right)}^{(j+1)} \mid \ell\left(X_{n+1}\right)=0 \text { and } \pi_{j}(\ell) \in \tilde{\Sigma}_{\left(d_{1}, \ldots, d_{j}\right)}^{(j)}\right\}
$$

and

$$
\Sigma_{\left(d_{1}, \ldots, d_{j}, d_{j}\right)}^{(j+1)}=\left\{\ell \in V_{\left(d_{1}, \ldots, d_{j}, d_{j}\right)}^{(j+1)} \mid \pi_{j}(\ell) \in \tilde{\Sigma}_{\left(d_{1}, \ldots, d_{j}\right)}^{(j)}\right\} .
$$


By recursion, we get the desired family of cross-sections for all the $\mathfrak{g}_{j}^{*}, 1 \leq j \leq n$. We finally put $\Omega_{\mathbf{d}}=\left(\sigma^{(n)}\right)^{-1}\left(\Sigma_{\mathbf{d}}^{(n)}\right)$.

After all these choices are made, we can build functions $q_{r}$ and $p_{r}$ by recursion as described above, choosing $g \in \Sigma^{(n)}$ as the base point for each orbit [2, Théorème 1.6]. Observe that at each step $j$ for which $d_{j+1}=d_{j}+1$, the equation (1.1) that defines the new function $q=q_{d_{j+1}}^{(j+1)}$ becomes

$$
\sigma^{(j)}\left(\exp \left(q(\ell) X_{j}\right) \pi_{j}(\ell)\right)=\pi_{j}\left(\sigma^{(j+1)}(\ell)\right) .
$$

These functions are now defined globally on $\Omega_{\mathbf{d}}$, and because the cross-section maps $\sigma^{(j)}$ are Borel, it follows quickly (see [2, proof of Théorème 1.6]) that $q=q_{d_{j+1}}^{(j+1)}$ is Borel, and hence that canonical coordinates at each step are Borel.

Observe however that the cross-sections and corresponding mappings $\sigma^{(j)}$ are constructed inductively and there seems to be no simple description of these objects. As a result, more precise properties of the coordinate functions remain obscure in general. Here are relatively simple examples illustrating this construction.

Example 1.1. Let $\mathfrak{g}=\operatorname{span}\left\{Z_{1}, Z_{2}, Y, X_{1}, X_{2}, A\right\}$, where

$\left[X_{j}, Y\right]=Z_{j},\left[A, Z_{1}+i Z_{2}\right]=(1+i)\left(Z_{1}+i Z_{2}\right)$ and $\left[A, X_{1}+i X_{2}\right]=(1+i)\left(X_{1}+i X_{2}\right)$.

We take as our basis $\left\{Z_{1}, Z_{2}, Y, X_{1}, X_{2}, A\right\}$, put $\ell=\left(z_{1}, z_{2}, y, x_{1}, x_{2}, a\right)$ and build the sequence of cross-sections. We get $\Sigma^{(m)}=\Sigma_{(0, \ldots, 0)}^{(m)}$ for $m=1,2,3$; then natural choices are

$$
\Sigma^{(4)}=\Sigma_{(0,0,0,1)}^{(4)} \cup \Sigma_{(0,0,0,0)}^{(4)}=\left\{\left(z_{1}, z_{2}, 0,0\right), z_{1} \neq 0\right\} \cup\left\{\left(0, z_{2}, y, x_{1}\right)\right\}
$$

and

$$
\begin{aligned}
\Sigma^{(5)} & =\Sigma_{(0,0,0,1,1)}^{(5)} \cup \Sigma_{(0,0,0,0,1)}^{(5)} \cup \Sigma_{(0,0,0,0,0)}^{(5)} \\
& =\left\{\left(z_{1}, z_{2}, 0,0, x_{2}\right), z_{1} \neq 0\right\} \cup\left\{\left(0, z_{2}, 0, x_{1}, 0\right), z_{2} \neq 0\right\} \cup\left\{\left(0,0, y, x_{1}, x_{2}\right)\right\} .
\end{aligned}
$$

Now, a natural choice for $\tilde{\Sigma}^{(5)}$ is for instance

$$
\begin{aligned}
\tilde{\Sigma}^{(5)}=\{(\cos \theta, \sin \theta, 0, & -\lambda \sin \theta, \lambda \cos \theta), \lambda \in \mathbb{R}\} \\
& \cup\{(0,0, y, \cos \theta, \sin \theta)\} \cup\{(0,0, y, 0,0)\} .
\end{aligned}
$$

Then we get

$$
\begin{gathered}
\left.\sigma^{(5)}\left(\tilde{\Sigma}^{(5)}\right)=\left\{\left(\cos \theta, \sin \theta, 0,0, \frac{\lambda}{\cos \theta}\right), \cos \theta \neq 0\right\} \cup\{0, \varepsilon, 0,-\lambda \varepsilon, 0), \varepsilon= \pm 1\right\} \\
\cup\{(0,0, y, \cos \theta, \sin \theta)\} \cup\{(0,0, y, 0,0)\}
\end{gathered}
$$

and

$$
\begin{aligned}
\Sigma^{(6)}= & \Sigma_{(0,0,0,1,1,2)}^{(6)} \cup \Sigma_{(0,0,0,0,1,2)}^{(6)} \cup \Sigma_{(0,0,0,0,0,1)}^{(6)} \cup \Sigma_{(0,0,0,0,0,0)}^{(6)} \\
= & \left.\left\{\left(\cos \theta, \sin \theta, 0,0, \frac{\lambda}{\cos \theta}, 0\right), \cos \theta \neq 0\right\} \cup\{0, \varepsilon, 0,-\lambda \varepsilon, 0,0), \varepsilon= \pm 1\right\} \\
& \cup\{(0,0, y, \cos \theta, \sin \theta, 0)\} \cup\{(0,0, y, 0,0, a)\} .
\end{aligned}
$$


Finally, we get the following canonical coordinates:

1. For $\Omega_{(0,0,0,1,1,2)}=\left\{z_{1} \cos \log \sqrt{z_{1}^{2}+z_{2}^{2}}+z_{2} \sin \log \sqrt{z_{1}^{2}+z_{2}^{2}} \neq 0\right\}$,

$$
\begin{aligned}
& p_{2}=a, \quad q_{2}=\log \sqrt{z_{1}^{2}+z_{2}^{2}}, \\
& p_{1}=\frac{x_{1} \cos \log \sqrt{z_{1}^{2}+z_{2}^{2}}+x_{2} \sin \log \sqrt{z_{1}^{2}+z_{2}^{2}}}{\sqrt{z_{1}^{2}+z_{2}^{2}}}, \\
& q_{1}=\frac{y \sqrt{z_{1}^{2}+z_{2}^{2}}}{z_{1} \cos \log \sqrt{z_{1}^{2}+z_{2}^{2}}+z_{2} \sin \log \sqrt{z_{1}^{2}+z_{2}^{2}}} .
\end{aligned}
$$

2. For $\Omega_{(0,0,0,0,1,2)}=\left\{z_{1} \cos \log \sqrt{z_{1}^{2}+z_{2}^{2}}+z_{2} \sin \log \sqrt{z_{1}^{2}+z_{2}^{2}}=0, z_{1}^{2}+z_{2}^{2}>0\right\}$, we have $z_{2} \cos \log \sqrt{z_{1}^{2}+z_{2}^{2}}-z_{1} \sin \log \sqrt{z_{1}^{2}+z_{2}^{2}}= \pm \sqrt{z_{1}^{2}+z_{2}^{2}}$ and

$$
\begin{aligned}
& p_{2}=a, \quad q_{2}=\log \sqrt{z_{1}^{2}+z_{2}^{2}}, \\
& p_{1}=\frac{x_{2} \cos \log \sqrt{z_{1}^{2}+z_{2}^{2}}-x_{1} \sin \log \sqrt{z_{1}^{2}+z_{2}^{2}}}{\sqrt{z_{1}^{2}+z_{2}^{2}}}, \quad q_{1}= \pm y .
\end{aligned}
$$

3. For $\Omega_{(0,0,0,0,0,1)}=\left\{z_{1}=z_{2}=0, x_{1}^{2}+x_{2}^{2} \neq 0\right\}$, there are no $p_{2}$, no $q_{2}$ and

$$
p_{1}=a, q_{1}=\log \sqrt{x_{1}^{2}+x_{2}^{2}}
$$

4. For $\Omega_{(0,0,0,0,0,0)}=\left\{z_{1}=z_{2}=x_{1}=x_{2}=0\right\}$, there are no $p_{2}$, no $q_{2}$, no $p_{1}$, and no $q_{1}$.

The set $\Omega_{(0,0,0,0,1,2)}$ is clearly a singular set for the functions $p_{1}$ and $q_{1}$ in the open union $\left\{z_{1}^{2}+z_{2}^{2} \neq 0\right\}$ of the two first sets. We shall see that this singularity can be removed by using a complex basis. This will allow us to define better cross-sections and more convenient coordinates. Here is another example.

Example 1.2. Let $\mathfrak{g}$ be the Lie algebra with a basis $\left\{Z_{1}, Z_{2}, Y_{1}, Y_{2}, X_{1}, X_{2}, A\right\}$ and relations

$$
\begin{aligned}
{\left[X_{1}+i X_{2}, Y_{1}+i Y_{2}\right] } & =0,\left[X_{1}+i X_{2}, Y_{1}-i Y_{2}\right]=(1-i)\left(Z_{1}-i Z_{2}\right), \\
{\left[A, X_{1}+i X_{2}\right] } & =(1+i)\left(X_{1}+i X_{2}\right),\left[A, Y_{1}+i Y_{2}\right]=(1+2 i)\left(Y_{1}+i Y_{2}\right), \\
{\left[A, Z_{1}+i Z_{2}\right] } & =(2+i)\left(Z_{1}+i Z_{2}\right) .
\end{aligned}
$$

Write $\ell=\left(z_{1}, z_{2}, y_{1}, y_{2}, x_{1}, x_{2}, a\right)$ where $z_{1}=\ell\left(Z_{1}\right)$, and so on. We keep our basis and get $\Sigma^{(m)}=\Sigma_{(0, \ldots, 0)}^{(m)}=\mathfrak{g}_{m}^{*}$ for $m \leqslant 4$ and

$$
\Sigma^{(5)}=\Sigma_{(0,0,0,0,1)}^{(5)} \cup \Sigma_{(0,0,0,0,0)}^{(5)}
$$

with

$$
\begin{aligned}
& \Sigma_{(0,0,0,0,1)}^{(5)}=\left\{\left(z_{1}, z_{2}, 0, \mu, 0\right), z_{1}-z_{2} \neq 0\right\} \cup\left\{(z, z, \mu, 0,0), z_{1}=z_{2}=z \neq 0\right\}, \\
& \Sigma_{(0,0,0,0,0)}^{(5)}=\left\{\left(0,0, y_{1}, y_{2}, x_{1}\right), z_{1}=z_{2}=0\right\} .
\end{aligned}
$$


We get the following parametrizations: if $z_{1}-z_{2} \neq 0$,

$$
\left\{\begin{aligned}
z_{1} & =z_{1}, z_{2}=z_{2}, \\
y_{1} & =\frac{1}{2}\left(z_{1}-z_{2}\right) q_{1}, \\
y_{2} & =\frac{1}{2}\left(z_{1}+z_{2}\right) q_{1}+\mu \\
x_{1} & =p_{1} .
\end{aligned}\right.
$$

If $z_{1}=z_{2}=z \neq 0$,

$$
\left\{\begin{array}{l}
z_{1}=z, z_{2}=z \\
y_{1}=\mu \\
y_{2}=\frac{1}{2}\left(z_{1}+z_{2}\right) q_{1}=z q_{1} \\
x_{1}=p_{1} .
\end{array}\right.
$$

Then

$$
\Sigma^{(6)}=\Sigma_{(0,0,0,0,1,2)}^{(6)} \cup \Sigma_{(0,0,0,0,0)}^{(6)}
$$

with

$$
\begin{aligned}
\Sigma_{(0,0,0,0,1,2)}^{(6)} & =\left\{\left(z_{1}, z_{2}, 0,0,0,0\right), z_{1} \neq z_{2}\right\} \cup\left\{(z, z, 0,0,0,0), z_{1}=z_{2}=z \neq 0\right\}, \\
\Sigma_{(0,0,0,0,0)}^{(6)} & =\left\{\left(0,0, y_{1}, y_{2}, x_{1}, x_{2}\right), z_{1}=z_{2}=0\right\} .
\end{aligned}
$$

We get the following parametrizations: if $z_{1} \neq z_{2}$,

$$
\left\{\begin{array}{l}
z_{1}=z_{1}, z_{2}=z_{2} \\
y_{1}=\frac{1}{2}\left(z_{1}-z_{2}\right) q_{1}-\frac{1}{2}\left(z_{1}+z_{2}\right) q_{2}, \\
y_{2}=\frac{1}{2}\left(z_{1}+z_{2}\right) q_{1}+\frac{1}{2}\left(z_{1}-z_{2}\right) q_{2} \\
x_{1}=p_{1}, x_{2}=p_{2} .
\end{array}\right.
$$

If $z_{1}=z_{2}=z \neq 0$,

$$
\left\{\begin{array}{l}
z_{1}=z, z_{2}=z, \\
y_{1}=-z q_{2}, \\
y_{2}=z q_{1}, \\
x_{1}=p_{1}, x_{2}=p_{2} .
\end{array}\right.
$$

Finally,

$$
\Sigma^{(7)}=\Sigma_{(0,0,0,0,1,2,3)}^{(7)} \cup \Sigma_{(0,0,0,0,0,0,1)}^{(7)} \cup \Sigma_{(0,0,0,0,0,0,0)}^{(7)}
$$

with

$$
\begin{gathered}
\Sigma_{(0,0,0,0,1,2,3)}^{(7)}=\{(\cos (\theta), \sin (\theta), 0,0,0,0,0), \cos (\theta) \neq \sin (\theta)\} \\
\cup\left\{ \pm\left(\frac{1}{\sqrt{2}}, \frac{1}{\sqrt{2}}, 0,0,0,0,0\right)\right\}, \\
\Sigma_{(0,0,0,0,0,0,1)}^{(7)}=\left\{\left(0,0, \cos (\theta), \sin (\theta), \lambda_{1}, \lambda_{2}, 0\right)\right\} \\
\cup\{(0,0,0,0, \cos (\theta), \sin (\theta), 0)\}, \\
\Sigma_{(0,0,0,0,0,0,0)}^{(7)}=\{(0,0,0,0,0,0, a)\} .
\end{gathered}
$$


We get the following parametrizations: if $e^{-\left(1+\frac{i}{2}\right) \log \left(z_{1}^{2}+z_{2}^{2}\right)}\left(z_{1}+i z_{2}\right)=e^{i \theta}$ and $\cos (\theta) \neq \sin (\theta)$,

$$
\left\{\begin{aligned}
z_{1}+i z_{2} & =e^{(2+i) q_{3}} e^{i \theta}, \\
y_{1}+i y_{2} & =e^{(1+2 i) q_{3}}\left(q_{1} \cos (\theta)+i q_{2} \sin (\theta)\right), \\
x_{1}+i x_{2} & =e^{(1+i) q_{3}}\left(p_{1}+i p_{2}\right), \\
a & =p_{3} .
\end{aligned}\right.
$$

If $e^{-\left(1+\frac{i}{2}\right) \log \left(z_{1}^{2}+z_{2}^{2}\right)}\left(z_{1}+i z_{2}\right)= \pm e^{i \frac{\pi}{4}}$,

$$
\left\{\begin{aligned}
z_{1}+i z_{2} & = \pm e^{i \frac{\pi}{4}} \\
y_{1}+i y_{2} & =e^{(1+2 i) q_{3}} \frac{ \pm 1}{\sqrt{2}}\left(q_{1}+i q_{2}\right) \\
x_{1}+i x_{2} & =e^{(1+i) q_{3}}\left(p_{1}+i p_{2}\right) \\
a & =p_{3}
\end{aligned}\right.
$$

If $z_{1}+i z_{2}=0$, and $y_{1}^{2}+y_{2}^{2} \neq 0$,

$$
\left\{\begin{aligned}
z_{1}+i z_{2} & =0 \\
y_{1}+i y_{2} & =e^{(1+2 i) q_{1}} e^{i \theta}, \\
x_{1}+i x_{2} & =e^{(1+i) q_{3}}\left(\lambda_{1}+i \lambda_{2}\right), \\
a & =p_{1} .
\end{aligned}\right.
$$

If $z_{1}+i z_{2}=0, y_{1}+i y_{2}=0$, and $x_{1}^{2}+x_{2}^{2} \neq 0$,

$$
\left\{\begin{aligned}
z_{1}+i z_{2} & =0 \\
y_{1}+i y_{2} & =0 \\
x_{1}+i x_{2} & =e^{(1+i) q_{1}} e^{i \theta} \\
a & =p_{1} .
\end{aligned}\right.
$$

We can invert these formulae to get the $p_{k}$ and the $q_{k}$. As above, we see that the distinction between the two first cases is irrelevant, except for one point: we exchange the jump indices in some intermediate computation. Moreover, since the only polynomial function $P\left(z_{1}, z_{2}\right)$ vanishing on the spirals

$$
z_{1}+i z_{2}= \pm e^{(2+i) q} e^{i \frac{\pi}{4}} \quad(q \in \mathbb{R})
$$

is $P=0$, the minimal, open layer is not an algebraic set.

This example illustrates a common feature of exponential groups. One of the subalgebras $\mathfrak{g}_{j}$ belonging to the chosen flag is not an ideal (in the above example $j=5)$, and for a class of $\ell \in \mathfrak{g}^{*}, \operatorname{dim}\left(\left(\mathfrak{g}_{j+1}\right)_{\ell}\right)+2=\operatorname{dim}\left(\left(\mathfrak{g}_{j-1}\right)_{\ell}\right)$, so that the dimension of the corresponding orbits jumps by four. We address this situation in Case 2 of our proofs in Sections 6 and 7 , there we see that by replacing $\mathfrak{g}_{j}$ by a variable ideal $\mathfrak{g}_{\mathcal{O}}$, we can simplify the construction and parametrize larger classes of orbits simultaneously. (For the preceding example, canonical coordinates for the entire class of orbits for which $z_{1}+i z_{2} \neq 0$ will be constructed.)

Now consider a non-trivial coadjoint orbit $\mathcal{O}$ for an exponential solvable Lie group $G$. Suppose that $c=\left(p_{1}, p_{2}, \ldots, p_{d}, q_{1}, q_{2}, \ldots, q_{d}\right)$ is a global Darboux chart on $\mathcal{O}$. Then one has the natural geometric polarization

$$
\ell \mapsto \mathcal{F}(\ell)=\operatorname{span}\left\{\partial_{p_{k}} \mid \ell, 1 \leq k \leq d\right\}
$$


Suppose that the chart $c$ is obtained by choosing a flag of subalgebras $\left(\mathfrak{g}_{j}\right)$ and carrying out the method of [2] as illustrated in the preceding examples. If each of these real subalgebras is an ideal in $\mathfrak{g}$, then it can be seen that the geometric polarization above coincides with the polarization coming from the real Vergne polarization

$$
\mathfrak{a}(\ell)=\sum_{t=1}^{n} \mathfrak{g}_{t}(\ell)
$$

That is, for each $\ell \in \mathcal{O}$,

$$
\mathcal{F}(\ell)=\xi_{\ell}^{\mathfrak{a}(\ell)}
$$

In [3], it was shown that this does not always hold in the exponential case. Let us recall that example here.

Example 1.3. Let $\mathfrak{g}$ be the Lie algebra with a basis $\{Z, Y, X, A\}$ and relations

$$
[X, Y]=Z,[A, X+i Y]=(1+i)(X+i Y),[A, Z]=2 Z \text {. }
$$

We keep our basis and get $\Sigma^{(2)}=\Sigma_{(0,0)}^{(2)}=\{(z, y)\}$ and

$$
\Sigma^{(3)}=\Sigma_{(0,0,1)}^{(3)} \cup \Sigma_{(0,0,0)}^{(3)}=\{(z, 0,0), z \neq 0\} \cup\{(0, y, x)\} .
$$

The parametrization is thus: if $z \neq 0$,

$$
\left\{\begin{array}{l}
z=z \\
y=z q_{1} \\
x=p_{1}
\end{array}\right.
$$

Also,

$$
\begin{aligned}
\Sigma^{(4)}= & \Sigma_{(0,0,1,2)}^{(4)} \cup \Sigma_{(0,0,0,1)}^{(4)} \cup \Sigma_{(0,0,0,0)}^{(4)} \\
= & \{(z, 0,0,0), z \neq 0\} \cup\left\{(0, \cos (\theta), \sin (\theta), 0), z=0, x^{2}+y^{2} \neq 0\right\} \\
& \cup\{(0,0,0, a), x=y=z=0\} .
\end{aligned}
$$

In the case $z \neq 0$, we get

$$
\left\{\begin{aligned}
z & =\varepsilon e^{2 q_{2}}, \quad \varepsilon= \pm 1, \\
x+i y & =e^{(1+i) q_{2}}\left(p_{1}+i \varepsilon q_{1}\right), \\
a & =p_{2},
\end{aligned}\right.
$$

while, if $z=0, x^{2}+y^{2} \neq 0$, we get

$$
\left\{\begin{aligned}
z & =0, \\
x+i y & =e^{(1+i) q_{1}} e^{i \theta}, \\
a & =p_{1} .
\end{aligned}\right.
$$

Finally, if $x=y=z=0$, we get

$$
\left\{\begin{aligned}
z & =0, \\
x+i y & =0, \\
a & =a .
\end{aligned}\right.
$$


In this example, let $\mathcal{O}$ be a coadjoint orbit whose elements satisfy $z \neq 0$. Note that for $\ell \in \mathcal{O}$, the real Vergne polarization corresponding to the flag $\left(\mathfrak{g}_{j}\right)$ is $\mathfrak{a}(\ell)=$ $\mathbb{R}$-span $\{Z, Y\}$, and that if $s \notin G_{3}$, then $s(\mathfrak{a}(\ell)) \neq \mathfrak{a}(s \ell)$. This observation alone shows (as does a direct calculation) that $\mathcal{F}(\ell) \neq \xi_{\ell}^{\mathfrak{a}(\ell)}$ on $\mathcal{O}$. In fact it can be shown that there is no real subalgebra map $\ell \mapsto \mathfrak{a}(\ell)$ on $\mathcal{O}$ for which $\mathcal{F}(\ell)=\xi_{\ell}^{\mathfrak{a}(\ell)}$ holds for all $\ell \in \mathcal{O}$.

On the other hand, suppose that instead we use the complex flag with $\mathfrak{s}_{1}=\mathbb{C} Z$, $\mathfrak{s}_{2}=\mathfrak{s}_{1}+\mathbb{C}(X+i Y)$, and $\mathfrak{s}_{3}=\mathfrak{s}_{2}+\mathbb{C}(X-i Y)$. Then the complex polarization $\mathfrak{h}(\ell)=\mathfrak{s}_{2}$ does satisfy $s(\mathfrak{h}(\ell))=\mathfrak{h}(s \ell)$ and our construction will yield construct complex canonical coordinates for which (now as subsets of $\left.T_{\ell}(\mathcal{O})_{\mathbb{C}}\right) \mathcal{F}(\ell)_{\mathbb{C}}=\xi_{\ell}^{\mathfrak{h}(\ell)}$. This example illustrates the general situation that we address in Case 3 of the proof of Theorem 7.2 for some $j, \mathfrak{g}_{j}$ is not an ideal, and $\mathfrak{g}_{j-1}(\ell)=\mathfrak{g}_{j+1}(\ell)$ (in the above example $j=2$ ).

\section{The Fine Stratification OF $\mathfrak{g}^{*}$}

In this section, we recall the partition defined in [5. We shall see that this partition is explicit, algebraic, and depends only upon the choice of a complex Jordan-Hölder basis for $\mathfrak{s}$. Moreover, it is tied directly to the corresponding Vergne polarization. We begin with a choice of ordered basis $\left\{Z_{1}, Z_{2}, \ldots, Z_{n}\right\}$ for $\mathfrak{s}$.

Definition 1 (Adaptable basis). An ordered basis $\left\{Z_{1}, Z_{2}, \ldots, Z_{n}\right\}$ for $\mathfrak{s}$ will be called "adaptable" if it satisfies the following conditions:

(i) For each $1 \leq j \leq n, \mathfrak{s}_{j}=\mathbb{C}$-span $\left\{Z_{1}, Z_{2}, \ldots, Z_{j}\right\}$ is an ideal in $\mathfrak{s}$.

(ii) If $\mathfrak{s}_{j} \neq \overline{\mathfrak{s}}_{j}$, then $\mathfrak{s}_{j+1}=\overline{\mathfrak{s}}_{j+1}$ and $Z_{j+1}=\bar{Z}_{j}$. Moreover, in this case there is $c_{j} \in \mathfrak{g}^{*}$ and $\alpha_{j} \in \mathbb{R} \backslash\{0\}$ such that

$$
\left[A, Z_{j}\right]=c_{j}(A)\left(1+i \alpha_{j}\right) Z_{j} \bmod \mathfrak{s}_{j-1}
$$

holds for all $A \in \mathfrak{g}$.

(iii) If $\mathfrak{s}_{j}=\overline{\mathfrak{s}}_{j}$ and $\mathfrak{s}_{j-1}=\overline{\mathfrak{s}}_{j-1}$, then $Z_{j} \in \mathfrak{g}$.

(iv) For some $p, 1 \leq p \leq n, \mathfrak{s}_{p}$ is the nilradical of $\mathfrak{s}$, and $\mathfrak{s}_{j}=\overline{\mathfrak{s}_{j}}$ for all $p \leq j \leq n$.

For any exponential Lie algebra $\mathfrak{g}$, it is well known that such a basis exists for $\mathfrak{s}=\mathfrak{g}_{\mathbb{C}}$. It is evident that for any $m$ with $\mathfrak{s}_{m}=\overline{\mathfrak{s}}_{m},\left\{Z_{1}, Z_{2}, \ldots, Z_{m}\right\}$ is an adaptable basis for $\mathfrak{s}_{m}=\left(\mathfrak{g}_{m}\right)_{\mathbb{C}}$; this fact will be crucial in what follows.

Of course if $\mathfrak{g}$ is completely solvable, then we can choose a real adaptable basis for $\mathfrak{g}$. However, note that in condition (ii) we allow for the possibility that $c_{j}$ is identically zero, so that we are not required to select a real basis even if $\mathfrak{g}$ is nilpotent. Thus the notion of adaptable basis is weaker than the notion of "good basis" used in [5] (and elsewhere). We shall see that the constructions of [5] go through with no additional difficulty with an adaptable basis in place.

Before describing the resulting partition, we set some notation associated to the basis above. Put $\mathfrak{s}_{0}=\{0\}, I=\left\{0 \leq j \leq n \mid \mathfrak{s}_{j}=\overline{\mathfrak{s}}_{j}\right\}$, and for each $1 \leq j \leq n$, set

$$
j^{\prime}=\max (\{0,1, \ldots, j-1\} \cap I), \quad j^{\prime \prime}=\min (\{j, j+1, \ldots, n\} \cap I) .
$$

Thus for each $j \geq 1, \mathfrak{s}_{j^{\prime}}=\mathfrak{s}_{j-1} \cap \overline{\mathfrak{s}}_{j-1}$ and $\mathfrak{s}_{j^{\prime \prime}}=\mathfrak{s}_{j}+\overline{\mathfrak{s}}_{j}$. We identify an element $\ell$ belonging to the complex dual $\mathfrak{s}^{*}$ with the $n$-tuple $\left(\ell_{1}, \ell_{2}, \ldots, \ell_{n}\right)$ where $\ell_{j}=\ell\left(Z_{j}\right)$, and we set $Z_{j}^{*}=(0,0, \ldots, 0,1,0, \ldots, 0)$ (where 1 is in the $j$-th position). We embed $\mathfrak{g}^{*}$ in $\mathfrak{s}^{*}$ in the natural way so that $\mathfrak{g}^{*}=\left\{\ell \in \mathfrak{s}^{*} \mid \ell(\bar{Z})=\overline{\ell(Z)}\right\}$. Note that for 
$\ell \in \mathfrak{g}^{*}$, if $j \notin I$, then $\ell_{j+1}=\bar{\ell}_{j}$. Let $\mu_{j}: G \rightarrow \mathbb{C}^{\times}=\mathbb{C} \backslash\{0\}$ be defined by $g Z_{j}^{*}=\mu_{j}(g) Z_{j}^{*} \bmod \mathfrak{s}_{j}^{\perp}$, and let $\gamma_{j}: \mathfrak{g} \rightarrow \mathbb{C}$ be the differential of $\mu_{j}$.

Definition 2 (Stratification). Let $\mathcal{P}$ be any partition of $\mathfrak{g}^{*}$. We shall say that $\mathcal{P}$ is an (orbital) stratification of $\mathfrak{g}^{*}$ if $\mathcal{P}$ satisfies both of the following:

(i) Each $\Omega$ belonging to $\mathcal{P}$ is $G$-invariant.

(ii) For each $\Omega \in \mathcal{P}$, the coadjoint orbits in $\Omega$ have the same dimension.

(iii) There is a linear ordering on $\mathcal{P}$ with the property that for each $\Omega \in \mathcal{P}$,

$$
\bigcup\left\{\Omega^{\prime} \mid \Omega^{\prime} \leq \Omega\right\}
$$

is a Zariski open subset of $\mathfrak{g}^{*}$.

The subsets $\Omega$ belonging to a stratification will be called layers.

The results stated in [5, Proposition 2.6, Theorem 2.8] provide us with a stratification of $\mathfrak{g}^{*}$, and in each layer, an explicit description of the space of coadjoint orbits. In this section, we shall be concerned with the first part of this stratification procedure. We summarize as follows.

(1) To each $\ell \in \mathfrak{g}^{*}$ there is associated a set $\mathbf{e}(\ell) \subset\{1,2, \ldots, n\}$ of "jump indices" defined by

$$
\mathbf{e}(\ell)=\left\{1 \leq j \leq n \mid \mathfrak{s}_{j} \not \subset \mathfrak{s}_{j-1}+\mathfrak{s}(\ell)\right\}
$$

Note that if $j \notin I$, then the set $\{j, j+1\}$ may contain 0,1 or 2 jump indices, and if in fact $\{j, j+1\}$ does contain a jump index, then $j$ itself must be a jump index. For a subset $\mathbf{e}$ of $\{1,2, \ldots, n\}$, the set $\Omega_{\mathbf{e}}=\left\{\ell \in \mathfrak{g}^{*} \mid \mathbf{e}(\ell)=\mathbf{e}\right\}$ is $G$-invariant, and the collection of non-empty $\Omega_{\mathbf{e}}$ is a stratification of $\mathfrak{g}^{*}$, which we shall call the "coarse stratification" of $\mathfrak{g}^{*}$.

(2) To each $\ell$ there is associated a "polarizing sequence" of subalgebras

$$
\mathfrak{s}=\mathfrak{h}_{0}(\ell) \supset \mathfrak{h}_{1}(\ell) \supset \cdots \supset \mathfrak{h}_{d}(\ell)
$$

and an index sequence pair $\mathbf{i}(\ell)=\left\{i_{1}<i_{2}<\cdots<i_{d}\right\}$ and $\mathbf{j}(\ell)=\left(j_{1}, j_{2}, \ldots, j_{d}\right)$, having values in $\mathbf{e}(\ell)$, defined by the recursive equations $(1 \leq k \leq d)$ :

$$
\begin{gathered}
i_{k}=\min \left\{1 \leq j \leq n \mid \mathfrak{s}_{j} \cap \mathfrak{h}_{k-1}(\ell) \not \subset \mathfrak{h}_{k-1}(\ell)^{\ell}\right\}, \\
\mathfrak{h}_{k}(\ell)=\left(\mathfrak{h}_{k-1}(\ell) \cap \mathfrak{s}_{i_{k}}\right)^{\ell} \cap \mathfrak{h}_{k-1}(\ell),
\end{gathered}
$$

and

$$
j_{k}=\min \left\{1 \leq j \leq n \mid \mathfrak{s}_{j} \cap \mathfrak{h}_{k-1}(\ell) \not \subset \mathfrak{h}_{k}(\ell)\right\} .
$$

The subalgebra $\mathfrak{h}(\ell)=\mathfrak{h}_{d}(\ell)$ is the Vergne polarization associated with the sequence $\left\{\mathfrak{s}_{j}\right\}: \mathfrak{h}(\ell)=\sum_{j} \mathfrak{s}_{j}(\ell)$. For each $k, i_{k}<j_{k}$, and $\mathbf{e}(\ell)$ is the disjoint union of the values of $\mathbf{i}(\ell)$ and $\mathbf{j}(\ell)$. Note that since $\mathbf{i}(\ell)$ must be increasing, it is determined by $\mathbf{e}(\ell)$ and $\mathbf{j}(\ell)$. For any splitting of $\mathbf{e}$ into such a sequence pair $(\mathbf{i}, \mathbf{j})$ we set $\Omega_{\mathbf{e}, \mathbf{j}}=\left\{\ell \in \Omega_{\mathbf{e}} \mid \mathbf{j}(\ell)=\mathbf{j}\right\}$. The collection of non-empty $\Omega_{\mathbf{e}, \mathbf{j}}$ is a stratification of $\mathfrak{g}^{*}$, which we shall call the "fine stratification" (following [7], wherein the term fine stratification was coined).

Now let us compare the preceding partition with the partition of [2]. Let $\mathfrak{g}=$ $\mathfrak{g}_{n} \supset \mathfrak{g}_{n-1} \supset \cdots \supset \mathfrak{g}_{0}=\{0\}$ be subalgebras of $\mathfrak{g}$ such that for each $1 \leq j \leq n$, if $j \in I$, then $\left(\mathfrak{g}_{j}\right)_{\mathbb{C}}=\mathfrak{s}_{j}$. Consider the sets $V_{\mathbf{d}}$ and $\Omega_{\mathbf{d}}$ defined in Section 1. If $\mathfrak{g}$ is completely solvable, then $V_{\mathbf{d}}$ is $G$-invariant, and hence $\Omega_{\mathbf{d}}=V_{\mathbf{d}}$. In this case, it is shown in [1] that the fine (as well as the ultrafine defined in Section 4) stratification refines the partition $\Omega_{\mathbf{d}}$. Referring to the examples in Section 1 we see that in the exponential case, this result no longer holds. 
Example 2.1. As in Example1.1 let $\mathfrak{g}=\operatorname{span}\left\{Z_{1}, Z_{2}, Y, X_{1}, X_{2}, A\right\}$, where $\left[X_{j}, Y\right]$ $=Z_{j},\left[A, Z_{1}+i Z_{2}\right]=(1+i)\left(Z_{1}+i Z_{2}\right)$, and $\left[A, X_{1}+i X_{2}\right]=(1+i)\left(X_{1}+i X_{2}\right)$. Choose the adaptable basis $\left(Z_{1}+i Z_{2}, Z_{1}-i Z_{2}, Y, X_{1}+i X_{2}, X_{1}-i X_{2}, A\right)$ of $\mathfrak{s}$. We get the following layers from the preceding, listed in order:

1. $\mathbf{e}=\{1,3,4,6\}, \mathbf{j}=(6,4)$ and

$\Omega_{1}=\Omega_{\mathbf{e}, \mathbf{j}}=\left\{\ell=\left(z_{1}+i z_{2}, z_{1}-i z_{2}, y, x_{1}+i x_{2}, x_{1}-i x_{2}, a\right) \mid z_{1}+i z_{2} \neq 0\right\}$.

2. $\mathbf{e}=\{4,6\}, \mathbf{j}=(6)$ and

$$
\Omega_{2}=\Omega_{\mathbf{e}, \mathbf{j}}=\left\{\ell=\left(0,0, y, x_{1}+i x_{2}, x_{1}-i x_{2}, a\right) \mid x_{1}+i x_{2} \neq 0\right\} .
$$

3. $\mathbf{e}=\emptyset, \mathbf{j}=\emptyset$ and

$$
\Omega_{3}=\Omega_{\mathbf{e}, \mathbf{j}}=\{\ell=(0,0, y, 0,0, a)\} .
$$

Then $\Omega_{1}$ is the union of the two first layers $\Omega_{\mathbf{d}}$, where $\mathbf{d}=(0,0,0,1,1,2)$ and $\mathbf{d}=(0,0,0,0,1,2)$.

Example 2.2. As in Example 1.2, let $\mathfrak{g}$ have the basis $\left\{Z_{1}, Z_{2}, Y_{1}, Y_{2}, X_{1}, X_{2}, A\right\}$ with relations

$$
\begin{aligned}
& {\left[X_{1}+i X_{2}, Y_{1}+i Y_{2}\right]=0,\left[X_{1}+i X_{2}, Y_{1}-i Y_{2}\right]=(1-i)\left(Z_{1}-i Z_{2}\right),} \\
& {\left[A, X_{1}+i X_{2}\right]=(1+i)\left(X_{1}+i X_{2}\right),\left[A, Y_{1}+i Y_{2}\right]=(1+2 i)\left(Y_{1}+i Y_{2}\right),} \\
& {\left[A, Z_{1}+i Z_{2}\right]=(2+i)\left(Z_{1}+i Z_{2}\right) .}
\end{aligned}
$$

Choose the adaptable basis $(Z, \bar{Z}, Y, \bar{Y}, X, \bar{X}, A)$ in $\mathfrak{s}$, where $Z=Z_{1}+i Z_{2}, Y=$ $Y_{1}+i Y_{2}, X=X_{1}+i X_{2}$. We get the following layers:

1. $\mathbf{e}=\{1,3,4,5,6,7\}, \mathbf{j}=(7,6,5)$, and

$$
\Omega_{\mathbf{e}, \mathbf{j}}=\{\ell=(z, \bar{z}, y, \bar{y}, x, \bar{x}, a) \mid z \neq 0\} .
$$

2. $\mathbf{e}=\{3,7\}, \mathbf{j}=(7)$, and

$$
\Omega_{\mathbf{e}, \mathbf{j}}=\{\ell=(0,0, y, \bar{y}, x, \bar{x}, a) \mid y \neq 0\} .
$$

3. $\mathbf{e}=\{5,7\}, \mathbf{j}=(7)$, and

$$
\Omega_{\mathbf{e}, \mathbf{j}}=\{\ell=(0,0,0,0, x, \bar{x}, a) \mid x \neq 0\} .
$$

4. $\mathbf{e}=\emptyset, \mathbf{j}=\emptyset$, and

$$
\Omega_{\mathbf{e}, \mathbf{j}}=\{\ell=(0,0,0,0,0,0, a)\} .
$$

Example 2.3. As in Example 1.3, let $\mathfrak{g}$ be the Lie algebra with a basis $\{Z, Y, X, A\}$ and relations

$$
[X, Y]=Z,[A, X+i Y]=(1+i)(X+i Y),[A, Z]=2 Z .
$$

We choose the adaptable basis $(Z, X+i Y, X-i Y, A)$; write $\ell=(z, x+i y, x-i y, a)$. We get the simple layering:

1. $\mathbf{e}=\{1,2,3,4\}, \mathbf{j}=(3,4)$, and

$$
\Omega_{\mathbf{e}, \mathbf{j}}=\{\ell(z, x+i y, x-i y, a) \mid z \neq 0\} .
$$

2. $\mathbf{e}=\{2,4\}, \mathbf{j}=(4)$, and

$$
\Omega_{\mathbf{e}, \mathbf{j}}=\{\ell=(0, x+i y, x-i y, a) \mid x+i y \neq 0\} .
$$

3. $\mathbf{e}=\emptyset, \mathbf{j}=\emptyset$, and

$$
\Omega_{\mathbf{e}, \mathbf{j}}=\{\ell=(0,0,0, a)\} .
$$




\section{Orthogonal OBJECts ASSOCIATED With Fine LAYERS}

In this section, we shall restate the constructions surrounding [5, Lemma 1.3] (see also [4, Lemma 1.2.1]) in a form that is suited to our purposes. First we itemize the possible ways that the basis elements $Z_{i_{k}}, 1 \leq k \leq d$, can be situated within the ordered basis as a whole. To this end, set $K_{0}=\left\{1 \leq k \leq d \mid i_{k} \in I\right.$ and $\left.i_{k}-1 \in I\right\}$; this is the set of subindices $k$ for which $Z_{i_{k}}$ is real. Note that in the completely solvable case, it is possible (but from our point of view here, not necessary) to choose a real adaptable basis, so that $K_{0}=K$. If $Z_{i_{k}}$ is not real, then either $i_{k} \notin I$ or $i_{k}-1 \notin I$. With this in mind, we list each of the possibilities below, following the notation of [5, page 248]:

$$
\begin{aligned}
& K_{0}=\left\{1 \leq k \leq d \mid i_{k}^{\prime \prime}-i_{k}^{\prime}=1\right\}, \\
& K_{1}=\left\{1 \leq k \leq d \mid i_{k} \notin I \text { and } i_{k}+1 \notin \mathbf{e}\right\}, \\
& K_{2}=\left\{1 \leq k \leq d \mid i_{k}-1 \in \mathbf{j} \backslash I\right\}, \\
& K_{3}=\left\{1 \leq k \leq d \mid i_{k} \notin I \text { and } i_{k}+1 \in \mathbf{j}\right\}, \\
& K_{4}=\left\{1 \leq k \leq d \mid i_{k} \notin I \text { and } i_{k}+1 \in \mathbf{i}\right\}, \text { and } \\
& K_{5}=\left\{1 \leq k \leq d \mid i_{k}-1 \in \mathbf{i} \backslash I\right\} .
\end{aligned}
$$

A few observations are in order here. First note that if $k \in K_{2}$, then $i_{k}-1=j_{h}$, where $1 \leq h<k$. Second, it is shown in [5, page 252] that if $k \in K_{3}$, then $i_{k}+1=j_{k}$. Third, note that the fact that $\mathbf{i}$ is an increasing sequence implies that if $k \in K_{4}$, then $i_{k}+1=i_{k+1}$, and $K_{5}=K_{4}+1$. It follows from these observations that $K$ is the disjoint union $K=K_{0} \cup K_{1} \cup K_{2} \cup K_{3} \cup K_{4} \cup K_{5}$.

We now proceed toward a restatement of [5, Lemma 1.3] that is suited to our purposes here. We begin with two important remarks.

First, the proof of [5, Lemma 1.3] includes the construction, for each $j \in \mathbf{e}$, of three $\mathfrak{s}$-valued functions of $\ell: Z_{j}(\ell), \rho\left(Z_{j}(\ell), \ell\right)$, and finally $Y_{k}(\ell)\left(X_{k}(\ell)\right)$ for $j=i_{k}$ $\left(j=j_{k}\right)$. The work of [5] is only concerned with the functions $Y_{k}(\ell)$ and $X_{k}(\ell)$, and so the statement of [5. Lemma 1.3] does not include the first two functions. However, it is the first two functions - $Z_{j}(\ell)$ and $\rho\left(Z_{j}(\ell), \ell\right)$ - that are crucial for this paper, and will be emphasized in our restatement below.

Next, recall that in [5] it is assumed that the basis $\left\{Z_{j}\right\}$ is chosen with the slightly stronger assumption that if $j \notin I$, then $c_{j} \neq 0$. As we have noted above, it is important for this paper that the possibility that $c_{j}=0$ be allowed. Indeed, the assumption that $c_{j} \neq 0$ is used in only one place in [5]: in the proof of [5, Lemma 1.3], for the case $k=1 \in K_{4}$, the assumption that $c_{i_{k}} \neq 0$ allows for a simplification of the proof. In the proof of the induction step, when $k>1$, one considers only Lie algebra elements belonging to the subalgebra $\mathfrak{h}_{k-1}(\ell)$, and it is certainly possible that $c_{i_{k}}$ vanishes on $\mathfrak{h}_{k-1}(\ell)$. Thus when the case $k \in K_{4}$ is considered in the induction step, the assumption that $c_{i_{k}} \neq 0$ is irrelevant and is not used. The proof of [5, Lemma 1.3] can therefore be carried out when the basis used is adaptable by the lengthening the argument for the case $k=1 \in K_{4}$ in accordance with the argument for the case $k \in K_{4}$ when $k>1$. Hence we have the following:

Lemma 3.1 ([5, Lemma 1.3]). Fix an adaptable basis for $\mathfrak{s}$ and let $\Omega=\Omega_{\mathbf{e}, \mathbf{j}}$ be a fine layer with $2 d>0$ the dimension of the $G$-orbits in $\Omega$. Then there is a canonical construction for the following. 
(1) For each $j \in \mathbf{e}$, a rational function $Z_{j}: \Omega \rightarrow \mathfrak{s}_{j^{\prime \prime}}$. More precisely there are regular rational functions $\alpha_{\epsilon, k}$ and $\beta_{\varepsilon, k}$ on $\Omega$ such that

$Z_{i_{k}}(\ell)=\beta_{1, k}(\ell) \mathfrak{R e} Z_{i_{k}}+\beta_{2, k}(\ell) \mathfrak{I m} Z_{i_{k}}$ and $Z_{j_{k}}(\ell)=\alpha_{1, k}(\ell) \mathfrak{R e} Z_{j_{k}}+\alpha_{2, k}(\ell) \mathfrak{I m} Z_{j_{k}}$.

(2) For each $1 \leq k \leq d$, rational functions $V_{k}: \Omega \rightarrow \mathfrak{s}$ and $U_{k}: \Omega \rightarrow \mathfrak{s}$, and

(3) a finite open covering $F=\left\{O_{\epsilon}\right\}$ of $\Omega$, and for each $O_{\epsilon} \in F$ and $j \in \mathbf{e}$, an explicit function $\phi_{j}^{\epsilon}: O_{\epsilon} \rightarrow \mathbb{T}$, that satisfy the following properties.

(i) For each $\ell \in \Omega, U_{k}(\ell) \in \mathfrak{s}_{j_{k}^{\prime \prime}} \backslash \mathfrak{s}_{j_{k}^{\prime}} \cap \mathfrak{s}_{i_{k}^{\prime}}^{\ell} \backslash \mathfrak{s}_{i_{k}^{\prime \prime}}^{\ell}$ and $V_{k}(\ell) \in \mathfrak{s}_{i_{k}^{\prime \prime}} \backslash \mathfrak{s}_{i_{k}^{\prime}} \cap \mathfrak{s}_{j_{k}^{\prime}} \backslash \mathfrak{s}_{j_{k}^{\prime \prime}}$.

(ii) $\ell\left[U_{h}(\ell), U_{k}(\ell)\right]=\ell\left[V_{h}(\ell), V_{k}(\ell)\right]=0,1 \leq h, k \leq d$.

(iii) $\ell\left[U_{h}(\ell), V_{k}(\ell)\right]=0$ if and only if $h \neq k, 1 \leq h, k \leq d$.

(iv) For each $1 \leq k \leq d$, set

$$
\mathfrak{m}_{k}(\ell)=\mathbb{C}-\operatorname{span}\left\{V_{1}(\ell), V_{2}(\ell), \ldots, V_{k}(\ell), U_{1}(\ell), U_{2}(\ell), \ldots, U_{k}(\ell)\right\},
$$

so that for each $\ell \in \Omega, \mathfrak{s}=\mathfrak{m}_{k}(\ell) \oplus \mathfrak{m}_{k}(\ell)^{\ell}$. For $Z \in \mathfrak{s}, \ell \in \Omega$, let $\rho_{k}(\cdot, \ell)$ be the projection of $\mathfrak{s}$ onto $\mathfrak{m}_{k}(\ell)^{\ell}$ parallel to $\mathfrak{m}_{k}(\ell)$, with $\rho_{0}(\cdot, \ell)$ the identity mapping. Then $V_{k}(\ell)=\rho_{k-1}\left(Z_{i_{k}}(\ell), \ell\right) U_{k}(\ell)=\rho_{k-1}\left(Z_{j_{k}}(\ell), \ell\right), 1 \leq k \leq d$.

(v) For $1 \leq k \leq d$, if $k \in K_{0} \cup K_{1} \cup K_{2}$, then $\mathfrak{h}_{k}(\ell)=\mathfrak{h}_{k-1}(\ell) \cap\left\{V_{k}(\ell)\right\}^{\ell}$ holds for each $\ell \in \Omega$. If $k \in K_{4}$, then $\mathfrak{h}_{k+1}(\ell)=\mathfrak{h}_{k-1}(\ell) \cap\left\{V_{k}(\ell), V_{k+1}(\ell)\right\}^{\ell}$ holds for each $\ell \in \Omega$.

(vi) For each $O_{\epsilon} \in F$ and $\ell \in O_{\epsilon}$, the elements $\phi_{j}^{\epsilon}(\ell)^{-1} Z_{j}(\ell), j \in \mathbf{e}$, belong to $\mathfrak{g}$.

Proof. We proceed by induction on $k, k=1,2, \ldots, d$. For $k=1$, set $V_{1}(\ell)=Z_{i_{1}}(\ell)$ and $U_{1}(\ell)=Z_{j_{1}}(\ell)$ (the definition of the functions $Z_{i_{1}}(\ell)$ and $Z_{j_{1}}(\ell)$ will be clear from what follows). Suppose then that $k>1$ and assume that we have all the objects of Lemma 3.1 up to $k-1$. We proceed to define the functions $Z_{i_{k}}(\ell)$ and $Z_{j_{k}}(\ell)$. For ease of notation set $Y_{1}=\mathfrak{R e} Z_{i_{k}}, Y_{2}=\mathfrak{I m} Z_{i_{k}}, X_{1}=\mathfrak{R e} Z_{j_{k}}$, and $X_{2}=\mathfrak{I m} Z_{j_{k}}$. We first construct $Z_{i_{k}}(\ell)$ belonging to the $\mathbb{C}$-span of $\left\{Y_{1}, Y_{2}\right\}$. Write

$$
Z_{i_{k}}(\ell)=\beta_{1, k}(\ell) Y_{1}+\beta_{2, k}(\ell) Y_{2}
$$

and $V_{k}(\ell)=\rho_{k-1}\left(Z_{i_{k}}(\ell), \ell\right)$. We must define the functions $\beta_{\epsilon, k}(\ell)$, and for this we consider the various cases for the subindex $k$.

$k \in K_{0}: \beta_{1, k}(\ell)=1$ and $\beta_{2, k}(\ell)=0$.

$k \in K_{1}: \beta_{1, k}(\ell)=\ell\left[\rho_{k-1}\left(Z_{j_{k}}, \ell\right), Y_{1}\right]$ and $\beta_{2, k}(\ell)=\ell\left[\rho_{k-1}\left(Z_{j_{k}}, \ell\right), Y_{2}\right]$.

$k \in K_{2}$ : here we put $i_{k}-1=j_{h}$; we have already defined

$$
Z_{j_{h}}(\ell)=\alpha_{1, h}(\ell) \mathfrak{R e} Z_{j_{h}}+\alpha_{2, h}(\ell) \mathfrak{I m} Z_{j_{h}},
$$

and we set $Z_{i_{k}}(\ell)=-\alpha_{2, h}(\ell) \mathfrak{R e} Z_{j_{h}}+\alpha_{1, h}(\ell) \mathfrak{I m} Z_{j_{h}}$. Hence

$$
\beta_{1, k}(\ell)=-\alpha_{2, h}(\ell) \text { and } \beta_{2, k}(\ell)=-\alpha_{1, h}(\ell) .
$$

(Of course, with our notation, $Y_{1}=\mathfrak{R e} Z_{j_{h}}$ and $Y_{2}=-\mathfrak{I m} Z_{j_{h}}$.)

$k \in K_{3}$ : we just put $\beta_{1, k}(\ell)=0$ and $\beta_{2, k}(\ell)=1$.

$k \in K_{4}$ : we shall define both $\beta_{\epsilon, k}(\ell)$ and $\beta_{\epsilon, k+1}(\ell)$, i.e. we define the $\beta_{\epsilon, k}(\ell)$ simultaneously for $k \in K_{4}$ and $k \in K_{5}$. It is not necessarily true here that $Z_{j_{k+1}}=$ $\bar{Z}_{j_{k}}$, but it is true that $j_{k+1}>j_{k}^{\prime}$. Accordingly this case splits into two subcases.

Subcase $(a): Z_{j_{k+1}}=\bar{Z}_{j_{k}}$. Here $\beta_{1, k}(\ell)=1$ and $\beta_{2, k}(\ell)=0$ so that $Z_{i_{k}}(\ell)=Y_{1}$. Below, we shall see how to define $\alpha_{\epsilon, k}(\ell)$ and $U_{k}(\ell)$. Suppose this is done; then put

$$
Z_{i_{k+1}}(\ell)=\beta_{1, k+1}(\ell) \mathfrak{R e} Z_{i_{k+1}}+\beta_{2, k+1}(\ell) \mathfrak{I m} Z_{i_{k+1}}=\beta_{1, k+1}(\ell) Y_{1}-\beta_{2, k+1}(\ell) Y_{2}
$$


by choosing

$$
\beta_{1, k+1}(\ell)=-\frac{\ell\left[U_{k}(\ell), Y_{2}\right]}{\ell\left[U_{k}(\ell), Y_{1}\right]} \text { and } \beta_{2, k+1}(\ell)=-1,
$$

so that $\ell\left[U_{k}(\ell), Z_{i_{k+1}}(\ell)\right]=0$.

It follows that $V_{k+1}(\ell)=\rho_{k}\left(Z_{i_{k+1}}(\ell), \ell\right)=\rho_{k-1}\left(Z_{i_{k+1}}(\ell), \ell\right)$. Note that one also has $V_{k+1}(\ell)=\rho_{k}\left(Y_{2}, \ell\right)$, but the definition of $Z_{i_{k+1}}(\ell)$ made here is more suited to our purposes.

Subcase $(b): Z_{j_{k+1}} \neq \bar{Z}_{j_{k}}$. In this case one has $j_{k+1}>j_{k}^{\prime \prime}$ ([5, page 250]). For the index $i_{k}$, this case is the same as $k \in K_{1}$ : one has

$$
\beta_{1, k}(\ell)=\ell\left[\rho_{k-1}\left(Z_{j_{k}}, \ell\right), Y_{1}\right] \text { and } \beta_{2, k}(\ell)=\ell\left[\rho_{k-1}\left(Z_{j_{k}}, \ell\right), Y_{2}\right] \text {. }
$$

As for the index $i_{k+1}$, we define

$$
Z_{i_{k+1}}(\ell)=-\beta_{2, k}(\ell) \mathfrak{R e} Z_{i_{k}}+\beta_{1, k}(\ell) \mathfrak{I m} Z_{i_{k}}
$$

so that

$$
\beta_{1, k+1}(\ell)=-\beta_{2, k}(\ell) \text { and } \beta_{2, k+1}(\ell)=-\beta_{1, k}(\ell) .
$$

Note that, in this subcase, because $j_{k+1}>j_{k}^{\prime \prime}$, we also have $V_{k+1}(\ell)=\rho_{k}\left(Z_{i_{k+1}}(\ell), \ell\right)$ $=\rho_{k-1}\left(Z_{i_{k+1}}(\ell), \ell\right)$.

For future reference we write $K_{4}=K_{4 a} \cup K_{4 b}$ and $K_{5}=K_{5 a} \cup K_{5 b}$ according to Subcases (a) and (b) above.

Now that we have defined $Z_{i_{k}}(\ell)$ and $V_{k}(\ell)$ for all possible cases, we must define $Z_{j_{k}}(\ell)$ belonging to the $\mathbb{C}$-span of $\left\{X_{1}, X_{2}\right\}$. Write

$$
Z_{j_{k}}(\ell)=\alpha_{1, k}(\ell) X_{1}+\alpha_{2, k}(\ell) X_{2} .
$$

It is shown in [4] that one formula for $\alpha_{\epsilon, k}(\ell)$ is sufficient, namely

$$
\alpha_{1, k}(\ell)=\ell\left[X_{1}, V_{k}(\ell)\right] \text { and } \alpha_{2, k}(\ell)=\ell\left[X_{2}, V_{k}(\ell)\right],
$$

or more explicitly,

$$
\alpha_{\epsilon, k}(\ell)=\beta_{1, k}(\ell) \ell\left[X_{\epsilon}, \rho_{k-1}\left(Y_{1}, \ell\right)\right]+\beta_{2, k}(\ell) \ell\left[X_{\epsilon}, \rho_{k-1}\left(Y_{2}, \ell\right)\right], \epsilon=1,2 .
$$

Note that if $j_{k}>p$ (that is, $Z_{j_{k}}$ lies outside the nilradical of $\mathfrak{s}$ ), then conditions (iii) and (iv) of the definition of adaptable basis say that $Z_{j_{k}}=X_{1} \in \mathfrak{g}$. In this case we simply set $\alpha_{1, k}(\ell)=1$ and $\alpha_{2, k}(\ell)=0$ so that $Z_{j_{k}}(\ell)=Z_{j_{k}}$.

The covering sets $O_{\epsilon}$ are defined as follows. For each $\epsilon=\left(\epsilon_{1}, \epsilon_{2}, \ldots, \epsilon_{d}\right) \in\{1,2\}^{d}$, set

$$
O_{\epsilon}=\left\{\ell \in \Omega \mid \beta_{\epsilon_{k}, k}(\ell) \neq 0, \quad 1 \leq k \leq d\right\} .
$$

The functions $\phi_{i_{k}}^{\epsilon}(\ell)$ are defined by

$$
\phi_{i_{k}}^{\epsilon}(\ell)=\frac{\beta_{\epsilon_{k}, k}(\ell)}{\left|\beta_{\epsilon_{k}, k}(\ell)\right|}, \quad 1 \leq k \leq d .
$$

If $j_{k} \leq p$, then put $\phi_{j_{k}}^{\epsilon}(\ell)=\phi_{i_{k}}^{\epsilon}(\ell)$, while if $j_{k}>p$, then put $\phi_{j_{k}}^{\epsilon}(\ell)=1$. The fact that $\phi_{j}^{\epsilon}(\ell)^{-1} Z_{j}(\ell)$ belongs to $\mathfrak{g}$ in all cases follows from the fact that

$$
\mathfrak{I m}\left(\beta_{1, k}(\ell) \overline{\beta_{2, k}(\ell)}\right)=0 ;
$$

hence $\mathfrak{I m}\left(\alpha_{1, k}(\ell) \overline{\alpha_{2, k}(\ell)}\right)=0$ also. The verification of the remaining properties of these objects is exactly as in [5. Lemma 1.3].

It follows from properties (ii) and (iii) of Lemma 3.1 that for each $\ell \in \Omega$, the image of $\mathfrak{m}_{d}(\ell)$ under $\xi_{\ell}$ is $T_{\ell}\left(\mathcal{O}_{\ell}\right)_{\mathbb{C}}$. Accordingly we make the following definition. 
Definition 3 (Orthogonal tangent vectors). Let $\Omega$ be a fine layer in $\mathfrak{g}^{*}$ for a chosen adaptable basis, and let $\ell \in \Omega$. The vectors $U_{k}(\ell), V_{k}(\ell)$ will be called the orthogonal tangent vectors to $\mathcal{O}_{\ell}$ at $\ell$.

From the definition of $\rho_{k}(\cdot, \ell)$ and the fact that the elements $U_{k}(\ell)$ and $V_{k}(\ell)$ belong to $\mathbb{C g}$, we see that for each $\ell \in \Omega$,

(a) $\rho_{k}(\bar{Z}, \ell)=\overline{\rho_{k}(Z, \ell)}, Z \in \mathfrak{s}$,

(b) $\rho_{k}$ satisfies the recursion formula for each $Z \in \mathfrak{s}$ :

$$
\rho_{k}(Z, \ell)=\rho_{k-1}(Z, \ell)-\frac{\ell\left[\rho_{k-1}(Z, \ell), U_{k}(\ell)\right]}{\ell\left[V_{k}(\ell), U_{k}(\ell)\right]} V_{k}(\ell)-\frac{\ell\left[\rho_{k-1}(Z, \ell), V_{k}(\ell)\right]}{\ell\left[U_{k}(\ell), V_{k}(\ell)\right]} U_{k}(\ell),
$$

and

(c) for any $W, Z \in \mathfrak{s}, \ell\left[\rho_{k}(W, \ell), \rho_{k}(Z, \ell)\right]=\ell\left[W, \rho_{k}(Z, \ell)\right]=\ell\left[\rho_{k}(W, \ell), Z\right]$.

We summarize several other useful properties of $\rho_{k}$ in the following lemma.

Lemma 3.2. One has each of the following:

(d) For $1 \leq j \leq n, \rho_{k}\left(\mathfrak{s}_{j}, \ell\right) \subset \mathfrak{s}_{j^{\prime \prime}}$.

(e) For $0 \leq k \leq d-1, \rho_{k}(\mathfrak{s}, \ell) \subset \mathfrak{s}_{i_{k+1}^{\ell}}^{\prime}$ and $\rho_{d}(\mathfrak{s}, \ell) \subset \mathfrak{s}^{\ell}$.

(f) If $k \notin K_{4}$, then $\mathfrak{m}_{k}(\ell)^{\ell} \subset \mathfrak{h}_{k}(\ell)$, and hence (by definition) $\rho_{k}(\cdot, \ell)$ maps $\mathfrak{s}$ into $\mathfrak{h}_{k}(\ell)$.

(g) For each $1 \leq k \leq d, \rho_{k-1}(\cdot, \ell)$ maps $\mathfrak{s}_{i_{k}^{\prime}}$ into $\mathfrak{s}^{\ell}$.

Proof. Part $(d)$ follows from the proof of [5, Lemma 1.3], while (e) follows from the fact that $\mathfrak{s}_{i_{k+1}^{\prime}} \subset \mathfrak{s}^{\ell}+\mathfrak{m}_{k}(\ell)$. Details are left to the reader.

Now suppose that $k \geq 1$ and that $(f)$ is true for subindices $h<k$ (the initial step of induction will be clear from what follows). First assume that $k \in K_{0} \cup K_{1} \cup K_{2}$. Then by part $(v)$ of Lemma 3.1 .

$$
\mathfrak{h}_{k}(\ell)=\mathfrak{h}_{k-1}(\ell) \cap\left\{V_{k}(\ell)\right\}^{\ell} .
$$

Since $k-1 \notin K_{4}$, we apply the induction hypothesis to get

$$
\begin{aligned}
\mathfrak{m}_{k}(\ell)^{\ell} & =\mathfrak{m}_{k-1}(\ell)^{\ell} \cap\left\{U_{k}(\ell), V_{k}(\ell)\right\}^{\ell} \\
& \subset \mathfrak{h}_{k-1}(\ell) \cap\left\{V_{k}(\ell)\right\}^{\ell}=\mathfrak{h}_{k}(\ell) .
\end{aligned}
$$

Suppose that $k \in K_{5}$. Then $k-2 \notin K_{4}$ and $k-1 \in K_{4}$, so again by part (v) of Lemma 3.1

$$
\mathfrak{h}_{k}(\ell)=\mathfrak{h}_{k-2}(\ell) \cap\left\{V_{k-1}(\ell), V_{k}(\ell)\right\}^{\ell},
$$

and the result follows much like the above.

If $k \in K_{3}$, then $k-1 \notin K_{4}$, so by induction and $\rho$-property (c) above, $U_{k}(\ell)$ and $V_{k}(\ell)$ belong to $\mathfrak{h}_{k-1}(\ell) \cap \mathfrak{s}_{i_{k}^{\prime \prime}}$, and by definition of $i_{k}$, it follows that

$$
\left(\mathfrak{h}_{k-1}(\ell) \cap \mathfrak{s}_{i_{k}^{\prime \prime}}\right)^{\ell} \supset\left(\mathfrak{h}_{k-1}(\ell) \cap \mathfrak{s}_{i_{k}^{\prime}}+\operatorname{span}\left\{V_{k}(\ell), U_{k}(\ell)\right\}\right)^{\ell} \supset \mathfrak{h}_{k-1}(\ell) \cap\left\{U_{k}(\ell), V_{k}(\ell)\right\}^{\ell} .
$$

Hence by induction and the above,

$$
\begin{aligned}
\mathfrak{m}_{k}(\ell)^{\ell} & =\mathfrak{m}_{k-1}(\ell)^{\ell} \cap\left\{U_{k}(\ell), V_{k}(\ell)\right\}^{\ell} \\
& \subset \mathfrak{h}_{k-1}(\ell) \cap\left\{U_{k}(\ell), V_{k}(\ell)\right\}^{\ell} \\
& \subset \mathfrak{h}_{k-1}(\ell) \cap\left(\mathfrak{h}_{k-1}(\ell) \cap \mathfrak{s}_{i_{k}^{\prime \prime}}\right)^{\ell} \\
& \subset \mathfrak{h}_{k-1}(\ell) \cap\left(\mathfrak{h}_{k-1}(\ell) \cap \mathfrak{s}_{i_{k}}\right)^{\ell}=\mathfrak{h}_{k}(\ell) .
\end{aligned}
$$


As for $(g)$, let $Z \in \mathfrak{s}_{i_{k}^{\prime}}$, and suppose first that $k-1 \notin K_{4}$. Then by definition of $i_{k}, \rho_{k-1}(Z, \ell) \in \mathfrak{h}_{k-1}(\ell) \cap \mathfrak{s}_{i_{k}-1} \subset \mathfrak{h}_{k-1}(\ell)^{\ell}$. Since

$$
\mathfrak{s}=\mathfrak{h}_{k-1}(\ell)+\operatorname{span}\left\{U_{1}(\ell), \ldots, U_{k-1}(\ell)\right\},
$$

then we have

$$
\rho_{k-1}(Z, \ell) \in \mathfrak{h}_{k-1}(\ell)^{\ell} \cap\left\{U_{1}(\ell), \ldots, U_{k-1}(\ell)\right\}^{\ell}=\mathfrak{s}^{\ell} .
$$

If $k-1 \in K_{4}$, then $i_{k}^{\prime}=i_{k-1}-1$, and so by definition of $i_{k-1}$,

$$
\rho_{k-1}(Z, \ell) \in \mathfrak{h}_{k-2}(\ell) \cap \mathfrak{s}_{i_{k-1}-1} \subset \mathfrak{h}_{k-2}(\ell)^{\ell} .
$$

Since $\mathfrak{s}=\mathfrak{h}_{k-2}(\ell)+\operatorname{span}\left\{U_{1}(\ell), \ldots, U_{k-2}(\ell)\right\}$, we obtain the result in a similar way.

Later we shall use the fact that several of the preceding functions on the fine layer $\Omega$, including the functions $Z_{j}(\ell)$, are invariant in the nilpotent case.

Lemma 3.3. Assume that $\mathfrak{g}$ is nilpotent. Let $\Omega$ be a fine layer whose orbits have dimension $2 d>0$. Let $k, 1 \leq k \leq d$, be a subindex such that $k \notin K_{5}$, let $X \in$ $\mathfrak{s}_{j_{k}^{\prime \prime}} \backslash \mathfrak{s}_{j_{k}^{\prime}}, Y \in \mathfrak{s}_{i_{k}^{\prime \prime}} \backslash \mathfrak{s}_{i_{k}^{\prime}}$, and set $\beta(\ell)=\ell\left[X, \rho_{k-1}(Y, \ell)\right], \ell \in \Omega$. Then $\beta$ is $G$-invariant on $\Omega$. In particular for each $\epsilon \in\{1,2\}^{d}, 1 \leq k \leq d, j \in \mathbf{e}$, the functions $\beta_{\epsilon, k}$ and $\alpha_{\epsilon, k}$ are $G$-invariant functions, and the covering set $O_{\epsilon}$ is a $G$-invariant subset of $\Omega$.

Proof. Suppose that $k=1$. For $s \in G$, we have $s^{-1} X=X+V$ where $V \in \mathfrak{s}_{j_{1}^{\prime}}$ and $s^{-1} Y=Y+W$ where $W \in \mathfrak{s}_{i_{1}^{\prime}}$. By definition of $i_{1}, j_{1}$ we have $W \in \mathfrak{s}^{\ell}$ and $V \in \mathfrak{h}_{1}(\ell)$. We claim that $\ell[V, Y]=0$. If $1 \in K_{0} \cup K_{1}$, then $\mathfrak{h}_{1}(\ell)=\mathfrak{s}_{i_{1}^{\prime \prime}}^{\ell}$, and our claim holds. If $1 \in K_{3}$, then $j_{1}^{\prime}=i_{1}^{\prime}$, so by definition of $i_{1}$, we have $V \in \mathfrak{s}^{\ell}$. Finally, if $1 \in K_{4}$, then we have $V \in \mathfrak{s}_{j_{1}^{\prime}} \cap \mathfrak{h}_{1}(\ell) \subset \mathfrak{h}_{2}(\ell)$ since $j_{2}>j_{1}^{\prime}$. But when $1 \in K_{4}$, one has $\mathfrak{h}_{2}(\ell)=\mathfrak{s}_{i_{1}^{\prime \prime}}^{\ell}$. Hence in all cases the claim holds.

Now it is immediate that

$$
\beta(s \ell)=\ell\left[s^{-1} X, s^{-1} Y\right]=\ell[X+V, Y+W]=\ell[X, Y]=\beta(\ell) .
$$

Suppose that $k>1$ and that the lemma holds for $1 \leq h \leq k-1$. We begin by showing that for each $\ell \in \Omega$ there is $Y(\ell) \in \mathfrak{s}_{i_{k}^{\prime \prime}}$ and $d(\ell) \in \mathbb{C}$ such that both $Y(\ell)$ and $d(\ell)$ are $G$-invariant and such that $\rho_{k-1}(Y, \ell)=d(\ell) Y(\ell) \bmod \mathfrak{s}_{i_{k}^{\prime}}$.

If $k \in K_{0} \cup K_{1} \cup K_{3} \cup K_{4}$, then the definition of $\rho_{k-1}$ shows that we can take $Y(\ell)=Y, d(\ell)=1$.

Suppose that $k \in K_{2}$, and set $i_{k}-1=j_{h}$ (for some $h$ such that $1 \leq h \leq k-1$ ). By induction the functions

$Z_{j_{h}}(\ell)=\alpha_{1, h}(\ell) \mathfrak{R e} Z_{j_{h}}+\alpha_{2, h}(\ell) \mathfrak{I m} Z_{j_{h}}$ and $\check{Z}_{j_{h}}(\ell)=-\alpha_{2, h}(\ell) \mathfrak{R e} Z_{j_{h}}+\alpha_{1, h}(\ell) \mathfrak{I m} Z_{j_{h}}$

are both $G$-invariant. For each $\ell \in \Omega$ we have complex numbers $c(\ell)$ and $d(\ell)$ such that $Y=c(\ell) Z_{j_{h}}(\ell)+d(\ell) \check{Z}_{j_{h}}(\ell)$, and it follows that $c(\ell)$ and $d(\ell)$ are $G$-invariant functions on $\Omega$. Now by definition of $\rho_{k-1}$ we have $\rho_{k-1}(Y, \ell)=d(\ell) \rho_{k-1}\left(\check{Z}_{j_{h}}(\ell), \ell\right)$, and since $\ell\left[\check{Z}_{j_{h}}(\ell), V_{h}(\ell)\right]=0$ it follows that

$$
\rho_{k-1}\left(\check{Z}_{j_{h}}(\ell), \ell\right)=d(\ell) \check{Z}_{j_{h}}(\ell) \bmod \mathfrak{s}_{i_{k}^{\prime}},
$$

so we take $Y(\ell)=\check{Z}_{j_{h}}(\ell)$ if $k \in K_{2}$. 
Now, for any $k \notin K_{5}$ and $s \in G$,

$$
\begin{aligned}
s^{-1} \rho_{k-1}(Y, s \ell) & =s^{-1}(d(s \ell) Y(s \ell))=d(\ell) Y(\ell) \bmod \mathfrak{s}_{i_{k}^{\prime}} \\
& =\rho_{k-1}(Y, \ell) \bmod \mathfrak{s}_{i_{k}^{\prime}},
\end{aligned}
$$

and hence we have

$$
s^{-1} \rho_{k-1}(Y, s \ell)=\rho_{k-1}(Y, \ell) \bmod \mathfrak{s}_{i_{k}^{\prime}} \cap \mathfrak{h}_{k-1}(\ell) .
$$

We have a similar equation for $X$ : indeed, if $\bar{Z}_{j_{k}} \neq Z_{j_{h}}$ holds for all $1 \leq h \leq k-1$, then the definition of $\rho_{k-1}$ gives $\rho_{k-1}(X, \ell)=X \bmod \mathfrak{s}_{j_{k}^{\prime}}$, while if $\bar{Z}_{j_{k}}=Z_{j_{h}}$ holds for some $1 \leq h \leq k-1$, then an argument similar to the above gives

$$
\rho_{k-1}(X, \ell)=d(\ell) \check{Z}_{j_{h}}(\ell) \bmod \mathfrak{s}_{j_{k}^{\prime}} .
$$

Hence in any case we have $X(\ell) \in \mathfrak{s}$ and $d(\ell) \in \mathbb{C}$ such that $\rho_{k-1}(X, \ell)=$ $d(\ell) X(\ell) \bmod \mathfrak{s}_{j_{k}^{\prime}}$, and the same calculation shows

$$
s^{-1} \rho_{k-1}(X, s \ell)=\rho_{k-1}(X, \ell) \bmod \mathfrak{s}_{j_{k}^{\prime}} \cap \mathfrak{h}_{k-1}(\ell) .
$$

Thus, there are $V \in \mathfrak{s}_{j_{k}^{\prime}} \cap \mathfrak{h}_{k-1}(\ell)$ and $W \in \mathfrak{s}_{i_{k}^{\prime}} \cap \mathfrak{h}_{k-1}(\ell)$ such that

$$
\beta(s \ell)=\ell\left[s^{-1} \rho_{k-1}(X, s \ell), s^{-1} \rho_{k-1}(Y, s \ell)\right]=\ell\left[\rho_{k-1}(X, \ell)+V, \rho_{k-1}(Y, \ell)+W\right] .
$$

By definition of $i_{k}$ we have $W \in \mathfrak{h}_{k-1}(\ell)^{\ell}$, and hence $\ell\left[\rho_{k-1}(X, \ell)+V, W\right]=0$. As for $V$, the definition of $j_{k}$ says $V \in \mathfrak{h}_{k}(\ell)$, and we argue exactly as in the case $k=1$ that $\ell\left[V, \rho_{k-1}(Y, \ell)\right]=0$. Thus $\beta(s \ell)=\beta(\ell)$.

Since we have put $Z_{j}(\ell)=Z_{j}$ when $j>p$, the following is immediate.

Corollary 3.1. Let $j$ be a value of the index sequence $\mathbf{j}$ and set $m=j^{\prime \prime}$. Then the function $\ell \mapsto Z_{j}(\ell)$ is $G_{m}$-invariant.

We observe that when the subindex set $K_{3}$ is non-empty, the Vergne polarization does not have a real form.

Lemma 3.4. Let $\mathfrak{h}=\mathfrak{h}_{d}(\ell)$ be the complex polarization associated with an adaptable basis. Then

$$
\mathfrak{h}=\mathfrak{h} \cap \overline{\mathfrak{h}}+\operatorname{span}\left\{\rho_{k-1}\left(Z_{i_{k}}, \ell\right) \mid k \in K_{3}\right\} .
$$

Proof. We show that

$$
\mathfrak{h}_{k}(\ell)=\mathfrak{h}_{k}(\ell) \cap \overline{\mathfrak{h}_{k}(\ell)}+\operatorname{span}\left\{\rho_{h-1}\left(Z_{i_{h}}, \ell\right) \mid h \in K_{3}, h \leq k\right\}
$$

holds for $k \notin K_{4}$. If $k=0$, then $\mathfrak{h}_{0}(\ell)=\mathfrak{s}$, and so the above is trivially true.

Suppose $k \in K_{0} \cup K_{1} \cup K_{2}$. We have $\mathfrak{h}_{k}(\ell)=\mathfrak{h}_{k-1}(\ell) \cap\left\{V_{k}(\ell)\right\}^{\ell}$, and since $V_{k}(\ell) \in \mathbb{C g}$,

$$
\mathfrak{h}_{k}(\ell) \cap \overline{\mathfrak{h}_{k}(\ell)}=\mathfrak{h}_{k-1}(\ell) \cap \overline{\mathfrak{h}_{k-1}(\ell)} \cap\left\{V_{k}(\ell)\right\}^{\ell} .
$$

Now by induction

$$
\mathfrak{h}_{k-1}(\ell)=\mathfrak{h}_{k-1}(\ell) \cap \overline{\mathfrak{h}_{k-1}(\ell)}+\operatorname{span}\left\{\rho_{h-1}\left(Z_{i_{h}}, \ell\right) \mid h \in K_{3}, h \leq k-1\right\} .
$$

Hence

$$
\begin{aligned}
\mathfrak{h}_{k}(\ell) & =\left(\mathfrak{h}_{k-1}(\ell) \cap \overline{\mathfrak{h}_{k-1}(\ell)}+\operatorname{span}\left\{\rho_{h-1}\left(Z_{i_{h}}, \ell\right) \mid h \in K_{3}, h \leq k-1\right\}\right) \cap\left\{V_{k}(\ell)\right\}^{\ell} \\
& =\mathfrak{h}_{k-1}(\ell) \cap \overline{\mathfrak{h}_{k-1}(\ell)} \cap\left\{V_{k}(\ell)\right\}^{\ell}+\operatorname{span}\left\{\rho_{h-1}\left(Z_{i_{h}}, \ell\right) \mid h \in K_{3}, h \leq k-1\right\} \\
& =\mathfrak{h}_{k}(\ell) \cap \overline{\mathfrak{h}_{k}(\ell)}+\operatorname{span}\left\{\rho_{h-1}\left(Z_{i_{h}}, \ell\right) \mid h \in K_{3}, h \leq k\right\} .
\end{aligned}
$$


Suppose $k \in K_{3}$. Here $\rho_{k-1}\left(Z_{j_{k}}, \ell\right)=\overline{\rho_{k-1}\left(Z_{i_{k}}, \ell\right)}$ and $\mathfrak{h}_{k}(\ell)=\mathfrak{h}_{k-1}(\ell) \cap$ $\left\{\rho_{k-1}\left(Z_{i_{k}}, \ell\right)\right\}^{\ell}$. Hence

$$
\mathfrak{h}_{k}(\ell) \cap \overline{\mathfrak{h}_{k}(\ell)}=\mathfrak{h}_{k-1}(\ell) \cap \overline{\mathfrak{h}_{k-1}(\ell)} \cap\left\{\rho_{k-1}\left(Z_{i_{k}}, \ell\right), \rho_{k-1}\left(Z_{j_{k}}, \ell\right)\right\}^{\ell} .
$$

Set $\mathcal{C}=\operatorname{span}\left\{\rho_{h-1}\left(Z_{i_{h}}, \ell\right) \mid h \in K_{3}, h \leq k-1\right\}$ for ease of notation. We invoke the induction hypothesis:

$$
\begin{aligned}
\mathfrak{h}_{k}(\ell) & =\left(\mathfrak{h}_{k-1}(\ell) \cap \overline{\mathfrak{h}_{k-1}(\ell)}+\mathcal{C}\right) \cap\left\{\rho_{k-1}\left(Z_{i_{k}}, \ell\right)\right\}^{\ell} \\
& =\mathfrak{h}_{k-1}(\ell) \cap \overline{\mathfrak{h}_{k-1}(\ell)} \cap\left\{\rho_{k-1}\left(Z_{i_{k}}, \ell\right)\right\}^{\ell}+\mathcal{C} \\
& =\mathfrak{h}_{k-1}(\ell) \cap \overline{\mathfrak{h}_{k-1}(\ell)} \cap\left\{\rho_{k-1}\left(Z_{i_{k}}, \ell\right), \rho_{k-1}\left(Z_{j_{k}}, \ell\right)\right\}^{\ell}+\mathbb{C} \rho_{k-1}\left(Z_{i_{k}}, \ell\right)+\mathcal{C} \\
& =\mathfrak{h}_{k}(\ell) \cap \overline{\mathfrak{h}_{k}(\ell)}+\operatorname{span}\left\{\rho_{h-1}\left(Z_{i_{h}}, \ell\right) \mid h \in K_{3}, h \leq k\right\} .
\end{aligned}
$$

Suppose $k \in K_{5}$. The proof here is similar to the case $k \in K_{0} \cup K_{1} \cup K_{2}$, using the induction hypothesis for $k-2$, since

$$
\mathfrak{h}_{k}(\ell)=\mathfrak{h}_{k-2}(\ell) \cap\left\{V_{k-1}(\ell), V_{k}(\ell)\right\}^{\ell} .
$$

Now we shall look at the geometric interest of the orthogonal tangent vectors $U_{k}(\ell)$ and $V_{k}(\ell)$. Let us now recall that if $\mathfrak{g}$ is a real Lie algebra, the bivector field $\Lambda$ on the dual $\mathfrak{g}^{*}$ of $\mathfrak{g}$ is such that, for any $X, Y$ in $\mathfrak{g}$ :

$$
\left\langle\Lambda_{\ell}, X \wedge Y\right\rangle=\ell[X, Y]
$$

is the Kostant bivector on $\mathfrak{g}^{*}$. This bivector defines the canonical Poisson structure on $\mathfrak{g}^{*}$, and the symplectic leaves for $\Lambda$ are exactly the coadjoint orbits.

Let $\left(X_{j}\right)$ be a basis for $\mathfrak{g}$. Write $\left[X_{i}, X_{j}\right]=\sum_{k} C_{i j}^{k} X_{k}$ and, for $\ell$ in $\mathfrak{g}^{*}, \ell_{j}=\ell\left(X_{j}\right)$. Then the Kostant bivector field satisfies

$$
\Lambda_{\ell}=\sum_{i, j} \ell\left[X_{i}, X_{j}\right] X_{i} \wedge X_{j}=\sum_{i, j} \ell\left[X_{i}, X_{j}\right] \frac{\partial}{\partial \ell_{i}} \wedge \frac{\partial}{\partial \ell_{j}}=\sum_{i, j, k} C_{i j}^{k} \ell_{k} \frac{\partial}{\partial \ell_{i}} \wedge \frac{\partial}{\partial \ell_{j}} .
$$

Of course, we can extend this definition to the complexification $\mathfrak{s}$ of $\mathfrak{g}$ in the natural way. For any complex basis $\left(Z_{j}\right)$ for $\mathfrak{s}$, we get

$$
\Lambda_{\ell}=\sum_{i j k} \ell\left[Z_{i}, Z_{j}\right] \frac{\partial}{\partial \ell_{i}} \wedge \frac{\partial}{\partial \ell_{j}}
$$

if $\ell_{j}=\ell\left(Z_{j}\right)$. for $\Lambda$.

Using the orthogonal tangent vectors $U_{k}(\ell), V_{k}(\ell)$ we get a very simple expression

Theorem 3.2 (Epression for the Kostant bivector). Let $G$ be an exponential Lie group with Lie algebra $\mathfrak{g}$. Choose an adaptable basis $\left(Z_{j}\right)$ for $\mathfrak{s}=\mathfrak{g}_{\mathbb{C}}$, and let $\Omega$ be a fine layer belonging to the corresponding fine stratification with $U_{k}(\ell), V_{k}(\ell)$, $1 \leqslant k \leqslant d$, the orthogonal tangent vectors. For each $k$ let us put

$$
c_{k}(\ell)=\ell\left[U_{k}(\ell), V_{k}(\ell)\right], \ell \in \Omega .
$$

Then for each $\ell \in \Omega$ we have

$$
\Lambda_{\ell}=\sum_{k=1}^{d} c_{k}(\ell)^{-1} \xi_{\ell}^{U_{k}(\ell)} \wedge \xi_{\ell}^{V_{k}(\ell)} .
$$


Proof. Let us fix $\ell$ in $\Omega$; complete the family $\left(U_{k}(\ell), V_{k}(\ell)\right)$ as a basis for $\mathfrak{s}$, by adding vectors $Z_{a}, 1 \leq a \leq n-2 d$ in $\mathfrak{s}(\ell)$. Put $u_{k}\left(\ell^{\prime}\right)=\ell^{\prime}\left(U_{k}(\ell)\right), v_{k}\left(\ell^{\prime}\right)=\ell^{\prime}\left(V_{k}(\ell)\right)$, $1 \leq k \leq d$, and $z_{a}\left(\ell^{\prime}\right)=\ell^{\prime}\left(Z_{a}\right), 1 \leq a \leq n-2 d$. Thus we have complex coordinates on $\mathfrak{s}^{*}$. By definition, in the point $\ell$, we get only

$$
\Lambda_{\ell}=\left.\left.\sum_{k} \ell\left[U_{k}(\ell), V_{k}(\ell)\right] \frac{\partial}{\partial u_{k}}\right|_{\ell} \wedge \frac{\partial}{\partial v_{k}}\right|_{\ell} .
$$

But a direct computation shows that for all $h$ and $a, \xi_{\ell}^{U_{k}(\ell)} u_{h}=\xi_{\ell}^{U_{k}(\ell)} z_{a}=0$ and that for $h \neq k, \xi_{\ell}^{U_{k}(\ell)} v_{h}=0$, while

$$
\xi_{\ell}^{U_{k}(\ell)} v_{k}=-c_{k}(\ell)
$$

Thus

Similarly,

$$
\xi_{\ell}^{U_{k}(\ell)}=-\left.c_{k}(\ell) \frac{\partial}{\partial v_{k}}\right|_{\ell} .
$$

This proves our theorem.

$$
\xi_{\ell}^{U_{k}(\ell)}=\left.c_{k}(\ell) \frac{\partial}{\partial u_{k}}\right|_{\ell}
$$

Example 3.1. We recall Example 2.1\} $\mathfrak{s}=\mathfrak{g}_{\mathbb{C}}=\operatorname{span}\{Z, \bar{Z}, Y, X, \bar{X}, A\}$, where $[X, Y]=Z,[A, Z]=(1+i) Z$, and $[A, X]=(1+i) X$. Recall that $A$ and $Y$ belong to $\mathfrak{g}$, while $X=X_{1}+i X_{2}$ and $Z=Z_{1}+i Z_{2}$. Note that the index for the nilradical is $p=5$. We put $\ell=\left(z_{1}+i z_{2}, z_{1}-i z_{2}, y, x_{1}+i x_{2}, x_{1}-i x_{2}, a\right)=(z, \bar{z}, y, x, \bar{x}, a)$. There are two layers with non-trivial orbits.

1. $\mathbf{e}=\{1,3,4,6\}, \mathbf{j}=(6,4)$. Here $1 \in K_{1}$, and $i_{1}=1, j_{1}=6$. Thus

$$
\left\{\begin{array}{l}
Z_{i_{1}}(\ell)=V_{1}(\ell)=\left(z_{1}-z_{2}\right) Z_{1}+\left(z_{1}+z_{2}\right) Z_{2}=\mathfrak{R e}((1-i) \bar{z} Z), \\
Z_{j_{1}}(\ell)=U_{1}(\ell)=A \quad\left(\text { since } j_{1}>p\right) .
\end{array}\right.
$$

Now $2 \in K_{0}$, and $i_{2}=3, j_{2}=4$. Thus

$$
\left\{\begin{aligned}
Z_{i_{2}}(\ell) & =Y, V_{2}(\ell)=\rho_{1}(Y, \ell)=Y, \\
Z_{j_{2}}(\ell) & =z_{1} X_{1}+z_{2} X_{2}=\mathfrak{R e}(\bar{z} X), \\
U_{2}(\ell) & =\rho_{1}(\mathfrak{R e}(\bar{z} X), \ell)=\mathfrak{R e}(\bar{z} X)-\frac{x_{2}\left(z_{1}-z_{2}\right)-x_{1}\left(z_{1}+z_{2}\right)}{2\left(z_{1}^{2}+z_{2}^{2}\right)} V_{1}(\ell) \\
& =\mathfrak{R e}(\bar{z} X)-\frac{1}{2} \mathfrak{R e}\left((1+i) \frac{x}{z}\right) \mathfrak{R e}((1-i) \bar{z} Z) .
\end{aligned}\right.
$$

Moreover,

$$
\begin{aligned}
& c_{1}(\ell)=\ell\left[U_{1}(\ell), V_{1}(\ell)\right]=2|z|^{2}, \\
& c_{2}(\ell)=\ell\left[U_{2}(\ell), V_{2}(\ell)\right]=|z|^{2} .
\end{aligned}
$$

2. $\mathbf{e}=\{4,6\}, \mathbf{j}=(6)$. Here $1 \in K_{1}$ and $i_{1}=4$, so

$$
\left\{\begin{array}{l}
Z_{i_{1}}(\ell)=V_{1}(\ell)=\left(x_{1}-x_{2}\right) X_{1}+\left(x_{1}+x_{2}\right) X_{2}=\mathfrak{R e}((1-i) \bar{x} X), \\
Z_{j_{1}}(\ell)=U_{1}(\ell)=A
\end{array}\right.
$$

Hence

$$
c_{1}(\ell)=\ell\left[U_{1}(\ell), V_{1}(\ell)\right]=2|x|^{2} .
$$


Example 3.2. Recall Example 2.2 $\mathfrak{s}=\operatorname{span}\{Z, \bar{Z}, Y, \bar{Y}, X, \bar{X}, A\}$ with $[X, Y]=0$, $[X, \bar{Y}]=(1-i) \bar{Z},[A, X]=(1+i) X,[A, Y]=(1+2 i) Y$, and $[A, Z]=(2+i) Z$. Recall that $Z=Z_{1}+i Z_{2}$, where $Z_{1}, Z_{2} \in \mathfrak{g}$, with similar notation for $X$ and $Y$, and write $\ell=(z, \bar{z}, y, \bar{y}, x, \bar{x}, a)$ with the obvious meaning. For ease of notation set $z^{\prime}=\frac{1+i}{2} z=z_{1}^{\prime}+i z_{2}^{\prime}$, so that $\ell[X, \bar{Y}]=2 \overline{z^{\prime}}$. There are three layers with non-trivial orbits.

1. $\mathbf{e}=\{1,3,4,5,6,7\}, \mathbf{j}=(7,6,5)$. Here $1 \in K_{1}, i_{1}=1$ and $j_{1}=7>p(p=6$ in this example), so

$$
\left\{\begin{aligned}
Z_{i_{1}}(\ell) & =V_{1}(\ell)=\left(2 z_{1}-z_{2}\right) Z_{1}+\left(z_{1}+2 z_{2}\right) Z_{2}=\mathfrak{R e}((2-i) \bar{z} Z), \\
Z_{j_{1}}(\ell) & =U_{1}(\ell)=A \\
c_{1}(\ell) & =5|z|^{2} .
\end{aligned}\right.
$$

Now $2 \in K_{4}$, and $i_{2}=3, i_{3}=4, j_{2}=6, j_{3}=5$. Thus we have

$$
\left\{\begin{aligned}
Z_{i_{2}}(\ell) & =Y_{1}, V_{2}(\ell)=\rho_{1}\left(Y_{1}, \ell\right)=Y_{1}-\frac{y_{1}-2 y_{2}}{5|z|^{2}} \mathfrak{R e}((2-i) \bar{z} Z), \\
Z_{j_{2}}(\ell) & =\mathfrak{R e}\left(\ell\left[X, V_{2}(\ell)\right] \bar{X}\right)=\mathfrak{R e}\left(z^{\prime} X\right), \\
U_{2}(\ell) & =\rho_{1}\left(\mathfrak{R e}\left(z^{\prime} X\right), \ell\right)=\mathfrak{R e}\left(z^{\prime} X\right)-\frac{\mathfrak{R e}\left(z^{\prime}(1+i) x\right)}{5|z|^{2}} \mathfrak{R e}((2-i) \bar{z} Z), \\
c_{2}(\ell) & =\frac{1}{2}|z|^{2}
\end{aligned}\right.
$$

and since $\ell\left[Y_{2}, U_{2}(\ell)\right]=0$,

$$
\left\{\begin{array}{l}
Z_{i_{3}}(\ell)=Y_{2}, V_{3}(\ell)=\rho_{2}\left(Y_{2}, \ell\right)=\rho_{1}\left(Y_{2}, \ell\right)=Y_{2}-\frac{2 y_{1}+y_{2}}{5|z|^{2}} \mathfrak{R e}((2-i) \bar{z} Z), \\
Z_{j_{3}}(\ell)=z_{2}^{\prime} X_{1}+z_{1}^{\prime} X_{2}=\mathfrak{I m}\left(z^{\prime} X\right), \\
U_{3}(\ell)=\rho_{1}\left(\mathfrak{I m}\left(z^{\prime} X\right), \ell\right)=\mathfrak{I m}\left(z^{\prime} X\right)--\frac{\mathfrak{I m}\left(z^{\prime}(1+i) x\right)}{5|z|^{2}} \mathfrak{R e}((2-i) \bar{z} Z), \\
c_{3}(\ell)=\frac{1}{2}|z|^{2}
\end{array}\right.
$$

2. $\mathbf{e}=\{3,7\}, \mathbf{j}=(7)$. Here $1 \in K_{1}, i_{1}=3$, so

$$
\left\{\begin{aligned}
Z_{i_{1}}(\ell) & =V_{1}(\ell)=\mathfrak{R e}((1-2 i) \bar{y} Y) \\
Z_{j_{1}}(\ell) & =U_{1}(\ell)=A \\
c_{1}(\ell) & =5|y|^{2}
\end{aligned}\right.
$$

3. $\mathbf{e}=\{5,7\}, \mathbf{j}=(7)$. Here $1 \in K_{1}, i_{1}=5$, so

$$
\left\{\begin{aligned}
Z_{i_{1}}(\ell) & =V_{1}(\ell)=\mathfrak{R e}((1-i) \bar{x} X) \\
Z_{j_{1}}(\ell) & =U_{1}(\ell)=A \\
c_{1}(\ell) & =2|x|^{2}
\end{aligned}\right.
$$

Example 3.3. Recall Example $2.3 \mathfrak{s}=\operatorname{span}\{Z, X+i Y, X-i Y, A\}$ with $[X, Y]=$ $Z,[A, X+i Y]=(1+i)(X+i Y),[A, Z]=2 Z$. We use the same notation for $\ell \in \mathfrak{g}^{*}$ as in the preceding example. There are two layers with non-trivial orbits. 
1. $\mathbf{e}=\{1,2,3,4\}, \mathbf{j}=(3,4)$. Here $1 \in K_{0}$ and $2 \in K_{3}$ :

$$
\left\{\begin{aligned}
Z_{i_{1}}(\ell) & =V_{1}(\ell)=Z, Z_{j_{1}}(\ell)=U_{1}(\ell)=A \\
c_{1}(\ell) & =2 z, \\
Z_{i_{2}}(\ell) & =Y, V_{2}(\ell)=\rho_{1}(Y, \ell)=Y-\frac{x+y}{2 z} Z, \\
Z_{j_{2}}(\ell) & =X, U_{2}(\ell)=\rho_{1}(X, \ell)=X-\frac{x-y}{2 z} Z, \\
c_{2}(\ell) & =z .
\end{aligned}\right.
$$

2. $\mathbf{e}=\{2,4\}, \mathbf{j}=(4)$. Here $1 \in K_{1}, i_{1}=2$ :

$$
\left\{\begin{aligned}
Z_{i_{1}}(\ell) & =V_{1}(\ell)=\mathfrak{R e}((1-i)(x-i y)(X+i Y)), \\
Z_{j_{1}}(\ell) & =U_{1}(\ell)=A, \\
c_{1}(\ell) & =2\left(x^{2}+y^{2}\right) .
\end{aligned}\right.
$$

If we were to have general coordinate functions $\left(\lambda_{h}, p_{k}, q_{k}\right)$, well defined on the minimal layer $\Omega$, such that $\lambda_{h}$ is invariant and for which the restrictions of $p_{k}, q_{k}$ to any orbit $\mathcal{O}$ in $\Omega$ give canonical coordinates for this orbit, then the Kostant bivector on $\Omega$ would simply be

$$
\Lambda=\sum_{k=1}^{d} \partial_{p_{k}} \wedge \partial_{q_{k}} .
$$

The preceding theorem did not give canonical coordinates but gives a similar and explicit rational expression for the restriction to any fine layer $\Omega$ of the Kostant bivector on $\mathfrak{g}^{*}$.

\section{Ultrafine LAYERING AND CROSS-SECTION}

Now fix a layer $\Omega=\Omega_{\mathbf{e}, \mathbf{j}}$ in the fine stratification. Let $\ell \in \Omega$ and $j \in \mathbf{e}$ and set $C_{j}=\operatorname{ker} \gamma_{j} \cap \mathfrak{g}$. Recalling the equivariant restriction map $\pi_{j^{\prime \prime}}: \mathfrak{g}^{*} \rightarrow \mathfrak{g}_{j^{\prime \prime}}^{*}=\mathfrak{g}^{*} / \mathfrak{g}_{j^{\prime \prime}}^{\perp}$, we set

$$
\mathcal{U}_{j}(\ell)=\pi_{j^{\prime \prime}}\left(\mathfrak{g}_{j^{\prime}}^{\perp}\right)
$$

and

$$
\mathcal{W}_{j}(\ell)=\mathbb{R} \pi_{j^{\prime \prime}}(\ell)+\mathcal{U}_{j}(\ell) .
$$

Note that $\mathcal{U}_{j}(\ell)_{\mathbb{C}}=\mathbb{C}$-span $\left\{Z_{j}^{*}, \overline{Z_{j}^{*}}\right\}$. Now $\mathfrak{g}_{j^{\prime}}^{\ell} \cap \mathfrak{g}$ and $\exp \left(\mathfrak{g}_{j^{\prime}}^{\ell} \cap \mathfrak{g}\right)$ act on $\mathfrak{g}_{j^{\prime \prime}}^{*}$ and $\mathcal{W}_{j}(\ell)$ is an invariant subspace for this action, so we have a homomorphism $\eta_{j}: \mathfrak{g}_{j^{\prime}}^{\ell} \cap \mathfrak{g} \rightarrow \operatorname{End}\left(\mathcal{W}_{j}(\ell)\right)$ whose kernel is the ideal $\mathfrak{g}_{j^{\prime \prime}}^{\ell} \cap C_{j}$ (see [ $\underline{\text {, proof of }}$ Proposition 5, part II]). Consider the real Lie algebra

$$
\mathfrak{b}_{j}(\ell)=\mathfrak{g}_{j^{\prime}}^{\ell} \cap \mathfrak{g} / \mathfrak{g}_{j^{\prime \prime}}^{\ell} \cap C_{j} ;
$$

we identify $\mathfrak{b}_{j}(\ell)$ with $\eta_{j}\left(\mathfrak{g}_{j^{\prime}}^{\ell} \cap \mathfrak{g}\right)$ and put $\mathfrak{a}_{j}(\ell)=\mathfrak{g}_{j^{\prime}}^{\ell} \cap C_{j} / \mathfrak{g}_{j^{\prime \prime}}^{\ell} \cap C_{j}$. It is easily seen that $\mathfrak{a}_{j}(\ell)$ is an abelian ideal in $\mathfrak{b}_{j}(\ell)$. As in loc. cit., the structure of $\mathfrak{b}_{j}(\ell)$ can be analyzed according to various cases whether $\mathfrak{a}_{j}(\ell)=\mathfrak{b}_{j}(\ell), \mathfrak{a}_{j}(\ell)=(0)$, or neither. A direct result of this analysis is the following fact.

Lemma 4.1. Suppose that $\mathfrak{b}_{j}(\ell) \neq \mathfrak{a}_{j}(\ell) \neq(0)$, and let $A \in \mathfrak{g}_{j^{\prime}}$ such that $B=$ $\eta_{j}(A) \notin \mathfrak{a}_{j}(\ell)$. Then the linear map adB on the complex space $\mathfrak{a}_{j}(\ell)_{\mathbb{C}}$ has eigenvalues $\gamma_{j}(A)$ and $\bar{\gamma}_{j}(A)$. 
Proof of the preceding lemma is contained in [8, proof of Proposition 5, part II]. We now examine more carefully certain implications of this analysis for jump indices.

Lemma 4.2. Let $j$ be an index belonging to $\mathbf{e}$.

(a) If $\mathfrak{a}_{j}(\ell)=(0)$, then

$j$ is a value of the sequence $\mathbf{i}, j-1 \in I$, and either $\left\{\begin{array}{l}j \in I, \text { or } \\ j \notin I \text { and } j+1 \notin \mathbf{e} \text {. }\end{array}\right.$

(b) If $\mathfrak{a}_{j}(\ell) \neq(0)$, then $\mathfrak{g}_{j^{\prime}}^{\ell}=\mathfrak{g}_{j^{\prime \prime}}^{\ell}+\mathfrak{g}_{j^{\prime}}^{\ell} \cap C_{j}$.

Proof. Consider an index $j_{k}$ which is a value of $\mathbf{j}$. We have $V_{k}(\ell) \in \mathfrak{s}_{j^{\prime}}^{\ell} \backslash \mathfrak{s}_{j^{\prime \prime}}^{\ell}$ but also $V_{k}(\ell) \in \mathfrak{s}_{i_{k}^{\prime \prime}}$. Since $i_{k}<j_{k}$ then $V_{k}(\ell) \in \mathfrak{s}_{j_{k}} \subset \operatorname{ker} \gamma_{j_{k}}$. Taking a covering set $O_{\epsilon}$ that contains $\ell$, we have that $Y_{k}(\ell):=\phi_{i_{k}}^{\epsilon}(\ell)^{-1} V_{k}(\ell)$ belongs to $\mathfrak{g}_{j^{\prime}}^{\ell} \cap C_{j} \backslash \mathfrak{g}_{j^{\prime \prime}}^{\ell} \cap C_{j}$, and hence $\mathfrak{a}_{j}(\ell) \neq(0)$.

Next observe that $j \in \mathbf{e}$ means $\mathfrak{g}_{j^{\prime}}^{\ell} \neq \mathfrak{g}_{j^{\prime \prime}}^{\ell}$, hence $\mathfrak{b}_{j}(\ell) \neq(0)$. But if $\mathfrak{a}_{j}(\ell)=(0)$, then $\gamma_{j} \neq 0$, and because of this and our choice of adaptable basis, we have that $\gamma_{j}$ is real if and only if $j^{\prime \prime}-j^{\prime}=1$. In fact $\operatorname{dim}\left(\mathfrak{b}_{j}(\ell)\right)=1$ in this case, and so

$$
\operatorname{Card}\left(\left\{j^{\prime}+1, j^{\prime \prime}\right\} \cap \mathbf{e}\right)=\operatorname{dim}\left(\mathfrak{g}_{j^{\prime}}^{\ell} / \mathfrak{g}_{j^{\prime \prime}}^{\ell}\right)=1 .
$$

Part (a) follows from these observations. Turning to part (b), observe that

$$
\begin{aligned}
\operatorname{dim}\left(\mathfrak{g}_{j^{\prime \prime}}^{\ell}+\left(\mathfrak{g}_{j^{\prime}}^{\ell} \cap C_{j}\right) / \mathfrak{g}_{j^{\prime \prime}}^{\ell}\right) & =\operatorname{dim}\left(\mathfrak{g}_{j^{\prime}}^{\ell} \cap C_{j} / \mathfrak{g}_{j^{\prime \prime}}^{\ell} \cap C_{j}\right) \\
& =\operatorname{dim}\left(\mathfrak{a}_{j}(\ell)\right) .
\end{aligned}
$$

If $\mathfrak{a}_{j}(\ell) \neq(0)$ and $\mathfrak{a}_{j}(\ell)=\mathfrak{b}_{j}(\ell)$, then $\mathfrak{a}_{j}(\ell)=\mathfrak{g}_{j^{\prime}}^{\ell} / \mathfrak{g}_{j^{\prime \prime}}^{\ell}$. On the other hand, if $\mathfrak{b}_{j}(\ell) \neq \mathfrak{a}_{j}(\ell) \neq(0)$, then it follows from Lemma 4.1 that $\operatorname{dim}\left(\mathfrak{a}_{j}(\ell)\right)=j^{\prime \prime}-j^{\prime}$. In either case $\operatorname{dim}\left(\mathfrak{a}_{j}(\ell)\right)=\operatorname{dim}\left(\mathfrak{s}_{j^{\prime}}^{\ell} / \mathfrak{s}_{j^{\prime \prime}}^{\ell}\right)$, and the result follows.

The following argument is essentially contained in the proofs of [5, Proposition 1.5, Proposition 1.8] and is included here for completeness.

Proposition 4.1. Let $\ell$ belong to the fine layer $\Omega_{\mathbf{e}, \mathbf{j}}$ and let $j=i_{k}$ be a value of the sequence $\mathbf{i}$. Then $\mathfrak{a}_{j}(\ell)=(0)$ if and only if $\gamma_{j}\left(U_{k}(\ell)\right) \neq 0$.

Proof. Let $O_{\epsilon}$ be a covering set containing $\ell$; we have $X_{k}^{\epsilon}(\ell):=\phi_{i_{k}}^{\epsilon}(\ell)^{-1} U_{k}(\ell) \in$ $\mathfrak{g}_{j^{\prime}}^{\ell} \backslash \mathfrak{g}_{j^{\prime \prime}}^{\ell}$ and $\eta_{j}\left(X_{k}^{\epsilon}(\ell)\right) \neq 0$ in $\mathfrak{b}_{j}(\ell)$. Hence if $\mathfrak{a}_{j}(\ell)=(0)$, then $X_{k}^{\epsilon}(\ell) \notin C_{j}$ and $\gamma_{j}\left(U_{k}(\ell)\right) \neq 0$.

On the other hand, suppose that $\mathfrak{a}_{j}(\ell) \neq(0)$. If $\mathfrak{a}_{j}(\ell)=\mathfrak{b}_{j}(\ell)$, then it is immediate that $X_{k}^{\epsilon}(\ell)$ belongs to $C_{j}$, and hence $\gamma_{j}\left(U_{k}(\ell)\right)=0$. Assume then that we have $\mathfrak{b}_{j}(\ell) \neq \mathfrak{a}_{j}(\ell) \neq(0)$. If $k \in K_{3}$, then $U_{k}(\ell) \in \mathfrak{s}_{j^{\prime \prime}}, \gamma_{j}\left(U_{k}(\ell)\right)=0$, so we may assume that $k \notin K_{3}$.

Let $A, X_{1}$, and $X_{2}$ be elements of $\mathfrak{g}_{j^{\prime}}^{\ell}$ such that $B=\eta_{j}(A) \in \mathfrak{b}_{j}(\ell) \backslash \mathfrak{a}_{j}(\ell)$, and $\mathfrak{a}_{j}(\ell)=\operatorname{span}\left\{Y_{1}, Y_{2}\right\}$ where $Y_{\epsilon}=\eta_{j}\left(X_{\epsilon}\right), \epsilon=1,2$. Write $\gamma_{j}(A)=\delta(1+i \alpha)$. We can choose $X_{1}$ and $X_{2}$ so that with $X=X_{1}+i X_{2} \in \mathfrak{s}_{j^{\prime}}^{\ell}$ we have $[B, Y]=\delta(1+i \alpha) Y$, and hence $[A, X]=\delta(1+i \alpha) X$ modulo $\mathfrak{g}_{j^{\prime \prime}}^{\ell} \cap C_{j}$. It follows that $X_{1}$ and $X_{2}$ belong to the nilradical of $\mathfrak{g}$. We now consider two cases.

Case 1. Suppose that $k \in K_{0} \cup K_{1} \cup K_{2}$. Then $\mathfrak{h}_{k}(\ell)=\mathfrak{h}_{k-1}(\ell) \cap V_{k}(\ell)^{\ell}$ and $V_{k}(\ell) \in \mathfrak{s}_{j^{\prime \prime}} \backslash \mathfrak{s}_{j^{\prime}}$. Hence there is some linear combination $W$ of $X_{1}$ and $X_{2}$ for which $\ell\left[W, V_{k}(\ell)\right] \neq 0$. Now by Lemma 3.2, $\mathfrak{g}_{j^{\prime}}^{\ell} \subset \mathfrak{h}_{k-1}(\ell)$, hence $W \in \mathfrak{h}_{k-1}(\ell) \backslash \mathfrak{h}_{k}(\ell)$, 
and if $\mathfrak{s}_{p}$ is the nilradical of $\mathfrak{s}, \mathfrak{s}_{p} \cap \mathfrak{h}_{k-1}(\ell) \not \subset \mathfrak{h}_{k}(\ell)$. By definition of $j_{k}$ this means $\mathfrak{s}_{j_{k}} \subset \mathfrak{s}_{p}$, and hence $\gamma_{j}\left(U_{k}(\ell)\right)=0$.

Case 2. Suppose that $k \in K_{4}$. Then $\mathfrak{h}_{k+1}(\ell)=\mathfrak{h}_{k-1}(\ell) \cap\left\{V_{k}(\ell), V_{k+1}\right\}^{\ell}$ and $Y_{k}(\ell), V_{k+1}(\ell)$ belong to $\mathfrak{s}_{j^{\prime \prime}} \backslash \mathfrak{s}_{j^{\prime}}$. By definition of $\mathfrak{h}_{k-1}(\ell), \mathfrak{s}_{j^{\prime}}^{\ell} \subset \mathfrak{h}_{k-1}(\ell)$, and by Lemma 4.2 part (b), $\mathfrak{g}_{j^{\prime}}^{\ell}=\mathfrak{g}_{j^{\prime \prime}}^{\ell}+\operatorname{span}\left\{X_{1}, X_{2}\right\}$. Hence we have $W_{1} \in \operatorname{span}\left\{X_{1}, X_{2}\right\}$ such that $W_{1} \in \mathfrak{h}_{k-1}$ and $\ell\left[Z_{j}, W_{1}\right] \neq 0$. It follows that $W_{1} \in \mathfrak{h}_{k-1}(\ell) \backslash \mathfrak{h}_{k}(\ell)$ and that $\mathfrak{s}_{j_{k}} \subset \mathfrak{s}_{p}$. Hence $\gamma_{j}\left(U_{k}(\ell)\right)=0$. Similarly we have $W_{2} \in \operatorname{span}\left\{X_{1}, X_{2}\right\}$ such that $\ell\left[Z_{j}, W_{2}\right]=0$ and $\ell\left[Z_{j+1}, W_{2}\right] \neq 0$, whence $W_{2} \in \mathfrak{h}_{k}(\ell) \backslash \mathfrak{h}_{k+1}(\ell)$, and $\mathfrak{s}_{j_{k+1}} \subset \mathfrak{s}_{p}$. It follows that $\gamma_{j}\left(U_{k+1}(\ell)\right)=0$.

Now for each $\ell$ belonging to the fine layer $\Omega_{\mathbf{e}, \mathbf{j}}$, we set

$$
\varphi(\ell)=\left\{j \in \mathbf{e} \mid \mathfrak{a}_{j}(\ell)=(0)\right\},
$$

and for each subset $\varphi$ of $\mathbf{e}$, define the subset $\Omega_{\mathbf{e}, \mathbf{j}, \varphi}$ of $\Omega_{\mathbf{e}, \mathbf{j}}$ by

$$
\Omega_{\mathbf{e}, \mathbf{j}, \varphi}=\left\{\ell \in \Omega_{\mathbf{e}, \mathbf{j}} \mid \varphi(\ell)=\varphi\right\} .
$$

It is shown in 5 that this refinement of the fine stratification is itself a stratification.

Definition 4 (Ultrafine stratification). The collection of non-empty subsets $\Omega_{\mathbf{e}, \mathbf{j}, \varphi}$ of $\mathfrak{g}^{*}$ will be called the "ultrafine stratification" of $\mathfrak{g}^{*}$.

We have seen that $\varphi(\ell)$ is contained in the set of $\mathbf{i}$-values. For each $j=i_{k}$ in $\mathbf{i}$, we put

$$
\mathbf{b}_{j}(\ell)=\frac{\gamma_{j}\left(U_{k}(\ell)\right)}{\ell\left[Z_{j}, U_{k}(\ell)\right]} .
$$

The following is proved in 5 .

Lemma 4.3 ([5, Proposition 1.8, Corollary 2.2]). The rational function $\mathbf{b}_{j}(\ell)$ is non-singular and $\mu_{j}^{-1}$-relatively invariant on $\Omega_{\mathbf{e}, \mathbf{j}, \varphi}$.

Recall that the constructions of Sections 23 and the preceding lemma are dependent upon the choice of an adaptable basis, the definition of which is more flexible than the complexified "good basis" of [5]. The two notions differ only in one respect: the condition "if $\bar{Z}_{j} \neq Z_{j}$ then $\gamma_{j}$ is not real-valued" in the definition of good basis is replaced here by the condition "if $\bar{Z}_{j} \neq Z_{j}$ then either $\gamma_{j}$ is not real-valued, or $\gamma_{j}=0$ ". The former condition is used only once in [5, in the proof of [5. Lemma 3.1], and as has already been pointed out, its use there is non essential. All subsequent results of [5] therefore follow with this slightly more general choice of adaptable basis.

In particular, let $\Omega=\Omega_{\mathbf{e}, \mathbf{j}, \varphi}$ be an ultrafine layer. As a result of [5. Proposition 2.6], we have an explicit cross-section $\Sigma=\Sigma_{\mathbf{e}, \mathbf{j}, \varphi}$ for coadjoint orbits in $\Omega$. We now give a description of this cross-section that is simpler and more explicit than [5, Proposition 2.7].

Theorem 4.2 (Description of the cross-section). Let $\mathfrak{g}$ be an exponential Lie algebra and choose an adaptable basis for the complexification $\mathfrak{s}$ of $\mathfrak{g}$. Let $\Omega=\Omega_{\mathbf{e}, \mathbf{j}, \varphi}$ be a layer belonging to the resulting ultrafine stratification of $\mathfrak{g}^{*}$. If $\mathbf{e}=\emptyset$, then the cross-section $\Sigma$ for $\Omega$ is $\Sigma=\Omega$. If $\mathbf{e} \neq \emptyset$, let $Z_{j}(\ell), j \in \mathbf{e}$, be the functions of Lemma 3.1, part (1). Then the cross-section $\Sigma$ for $\Omega$ is given by

$$
\Sigma=\left\{\ell \in \Omega \mid \ell\left(Z_{j}(\ell)\right)=0 \text {, for all } j \in \mathbf{e} \backslash \varphi \text {, and }\left|\mathbf{b}_{j}(\ell)\right|=1 \text { for all } j \in \varphi\right\} \text {. }
$$


Proof. If $\mathbf{e}=\emptyset$, there is nothing to prove. Suppose $\mathbf{e} \neq \emptyset$. Referring to the description of $\Sigma$ given in [5, Proposition 2.7], we must show for all $\ell \in \Omega$ each of the following:

(1) If $j \in I$ and $j \notin \varphi$, then $\ell\left(Z_{j}(\ell)\right)=0$ is equivalent to the equation $\ell_{j}=0$.

(2) If $j \notin I$ and $j+1 \in \mathbf{e}$ also, then the system of equations

$$
\ell\left(Z_{j}(\ell)\right)=0, \ell\left(Z_{j+1}(\ell)\right)=0
$$

is equivalent to the equation $\ell_{j}=0$.

(3) If $j \notin I, j+1 \notin \mathbf{e}, j=i_{k} \in \mathbf{i}$, and $j \notin \varphi$, then

$$
\ell\left(Z_{j}(\ell)\right)=\ell\left[\rho_{k-1}\left(Z_{j_{k}}, \ell\right), \mathfrak{R e} Z_{j}\right] \mathfrak{R e} \ell_{j}+\ell\left[\rho_{k-1}\left(Z_{j_{k}}, \ell\right), \mathfrak{I m} Z_{j}\right] \mathfrak{I m} \ell_{j} .
$$

(4) If $j \notin I, j+1 \notin \mathbf{e}$, and $j=j_{k} \in \mathbf{j}$ (hence again $j \notin \varphi$ ), then the equation $\ell\left(Z_{j}(\ell)\right)=0$ is equivalent to the system of equations

$$
\begin{aligned}
& \mathfrak{R e}\left(\ell\left[\rho_{k-1}\left(\bar{Z}_{j}, \ell\right), \mathfrak{R e} Z_{i_{k}}\right] \ell_{j}\right)=0, \\
& \mathfrak{R e}\left(\ell\left[\rho_{k-1}\left(\bar{Z}_{j}, \ell\right), \mathfrak{I m} Z_{i_{k}}\right] \ell_{j}\right)=0 .
\end{aligned}
$$

(Note that the above corrects a misprint in the statement (ii), proof of Proposition 2.7 in [5.)

Statement (1) in the case that $j=i_{k}$ is clear since $\ell\left(Z_{j}(\ell)\right)=\ell_{j}$ (here $\left.k \in K_{0}\right)$. In the case that $j=j_{k}$, we have $\ell\left(Z_{j_{k}}(\ell)\right)=\ell\left[Z_{j_{k}}, V_{k}(\ell)\right] \ell_{j_{k}}$, and since $\ell\left[Z_{j_{k}}, V_{k}(\ell)\right] \neq$ 0 , then statement (1) follows.

As for statement (2), suppose first that $j=i_{k} \in \mathbf{i}$. Then either $j+1=j_{k}$ or $j+1=i_{k+1}$. If $j+1=j_{k}$, then

$$
\ell\left(Z_{j}(\ell)\right)=\mathfrak{I m} \ell_{j} \text { and } \ell\left(Z_{j+1}(\ell)\right)=\ell\left[\mathfrak{R e} Z_{j}, \rho_{k-1}\left(\mathfrak{I m} Z_{j}, \ell\right)\right] \mathfrak{R e} \ell_{j},
$$

so (2) is clear in this case. If $j+1=i_{k+1}$, then if $k \in K_{4 a}$,

$$
\ell\left(Z_{j}(\ell)\right)=\mathfrak{R e} \ell_{j} \text { and } \ell\left(Z_{j+1}(\ell)\right)=\mathfrak{I m} \ell_{j},
$$

while if $k \in K_{4 b}$,

$$
\begin{aligned}
\ell\left(Z_{j}(\ell)\right) & =\beta_{1, k}(\ell) \mathfrak{R e} \ell_{j}+\beta_{2, k}(\ell) \mathfrak{I m} \ell_{j}, \\
\ell\left(Z_{j+1}(\ell)\right) & =-\beta_{2, k}(\ell) \mathfrak{R e} \ell_{j}+\beta_{1, k}(\ell) \mathfrak{I m} \ell_{j} .
\end{aligned}
$$

In either case it is easily seen that the system in question is equivalent to the equation $\ell_{j}=0$.

Suppose next that $j \in \mathbf{j}$. If $j+1=i_{k}$, then

$$
\begin{aligned}
\ell\left(Z_{j}(\ell)\right) & =\alpha_{1, h}(\ell) \mathfrak{R e} \ell_{j}+\alpha_{2, h}(\ell) \mathfrak{I m} \ell_{j}, \\
\ell\left(Z_{j+1}(\ell)\right) & =-\alpha_{2, h}(\ell) \mathfrak{R e} \ell_{j}+\alpha_{1, h}(\ell) \mathfrak{I m} \ell_{j},
\end{aligned}
$$

and the result follows easily. If on the other hand $j+1 \in \mathbf{j}$ also, then write $\{j, j+1\}=\left\{j_{h}, j_{k}\right\}$ where $h<k$. For ease of notation set $X=Z_{j_{h}}, X=X_{1}+i X_{2}$. We have

$$
\begin{aligned}
& \ell\left(Z_{j_{h}}(\ell)\right)=a_{1,1}(\ell) \mathfrak{R e} \ell_{j}+a_{2,1}(\ell) \mathfrak{I m} \ell_{j}, \\
& \ell\left(Z_{j_{k}}(\ell)\right)=a_{1,2}(\ell) \mathfrak{R e} \ell_{j}+a_{2,2}(\ell) \mathfrak{I m} \ell_{j},
\end{aligned}
$$

where $a_{\epsilon, 1}(\ell)=\ell\left[X_{\epsilon}, V_{h}(\ell)\right]=\alpha_{\epsilon, h}(\ell)$ and $a_{\epsilon, 2}=\ell\left[X_{\epsilon}, V_{k}(\ell)\right], \epsilon=1$, 2. We must show that $a_{1,1} a_{2,2}-a_{1,2} a_{2,1}$ is non-vanishing on $\Omega$, and an easy calculation shows that this is equivalent to

$$
\ell\left[X, V_{h}(\ell)\right] \ell\left[\bar{X}, V_{k}(\ell)\right]-\ell\left[\bar{X}, V_{h}(\ell)\right] \ell\left[X, V_{k}(\ell)\right] \neq 0, \ell \in \Omega .
$$


Set

$$
\check{Z}_{j_{h}}(\ell)=-\alpha_{2, h}(\ell) X_{1}+\alpha_{1, h}(\ell) X_{2}=\frac{1}{2 i}\left(\ell\left[\bar{X}, V_{h}(\ell)\right] X-\ell\left[X, V_{h}(\ell)\right] \bar{X}\right) ;
$$

we need to show that $\ell\left[\check{Z}_{j_{h}}(\ell), V_{k}(\ell)\right] \neq 0$. Since

$$
\ell\left[Z_{j_{k}}(\ell), V_{k}(\ell)\right]=\ell\left[U_{k}(\ell), V_{k}(\ell)\right] \neq 0
$$

it will be enough to show that for each $\ell$,

$$
\check{Z}_{j_{h}}(\ell)=C(\ell) Z_{j_{k}}(\ell)
$$

holds for some (non-vanishing) complex number $C(\ell)$.

Set $a_{1}=\ell\left[\bar{X}, V_{h}(\ell)\right], a_{2}=\ell\left[X, V_{h}(\ell)\right]$, and

$$
c_{1}=\ell\left[\rho_{k-1}\left(\check{Z}_{j_{h}}(\ell), \ell\right), \bar{Z}_{i_{k}}\right], \quad c_{2}=\ell\left[\rho_{k-1}\left(\check{Z}_{j_{h}}(\ell), \ell\right), Z_{i_{k}}\right] .
$$

We have $a_{1} X=Z_{j_{h}}(\ell)+i \check{Z}_{j_{h}}(\ell)$ and $a_{2} \bar{X}=Z_{j_{h}}(\ell)-i \check{Z}_{j_{h}}(\ell)$, and since

$$
\rho_{k-1}\left(Z_{j_{h}}(\ell), \ell\right)=\rho_{k-1}\left(\rho_{h-1}\left(Z_{j_{h}}(\ell), \ell\right), \ell\right)=\rho_{k-1}\left(U_{h}(\ell), \ell\right)=0,
$$

then we have

$$
\rho_{k-1}(X, \ell)=\frac{1}{a_{1}} \rho_{k-1}\left(\check{Z}_{j_{h}}(\ell), \ell\right)
$$

and

$$
\rho_{k-1}(\bar{X}, \ell)=\frac{-i}{a_{1}} \rho_{k-1}\left(\check{Z}_{j_{h}}(\ell), \ell\right) .
$$

Hence

$$
\begin{aligned}
Z_{i_{k}}(\ell) & =\frac{1}{2}\left(\ell\left[\rho_{k-1}\left(Z_{j_{k}}, \ell\right), \bar{Z}_{i_{k}}\right] Z_{i_{k}}+\ell\left[\rho_{k-1}\left(Z_{j_{k}}, \ell\right), Z_{i_{k}}\right] \bar{Z}_{i_{k}}\right) \\
& =\frac{1}{2}\left(\ell\left[\rho_{k-1}(\bar{X}, \ell), \bar{Z}_{i_{k}}\right] Z_{i_{k}}+\ell\left[\rho_{k-1}(\bar{X}, \ell), Z_{i_{k}}\right] \bar{Z}_{i_{k}}\right) \\
& =\frac{1}{2}\left(\frac{-i}{a_{2}} c_{1} Z_{i_{k}}-\frac{i}{a_{2}} c_{2} Z_{i_{k}}\right)
\end{aligned}
$$

and

$$
\ell\left[\rho_{k-1}\left(\check{Z}_{j_{h}}(\ell), \ell\right), Z_{i_{k}}(\ell)\right]=\frac{c_{1} c_{2}}{i a_{2}} .
$$

We substitute into the definition of $Z_{j_{k}}(\ell)$ :

$$
\begin{aligned}
Z_{j_{k}}(\ell) & =\frac{1}{2}\left(\ell\left[\rho_{k-1}\left(\bar{Z}_{j_{k}}, \ell\right), Z_{i_{k}}(\ell)\right] Z_{j_{k}}+\ell\left[\rho_{k-1}\left(Z_{j_{k}}, \ell\right), Z_{i_{k}}(\ell)\right] \bar{Z}_{j_{k}}\right) \\
& =\frac{1}{2}\left(\ell\left[\rho_{k-1}(X, \ell), Z_{i_{k}}(\ell)\right] \bar{X}+\ell\left[\rho_{k-1}(\bar{X}, \ell), Z_{i_{k}}(\ell)\right] X\right) \\
& =\frac{1}{2}\left(\frac{i}{a_{1}} \ell\left[\rho_{k-1}\left(\check{Z}_{j_{h}}(\ell), \ell\right), Z_{i_{k}}(\ell)\right] \bar{X}+\frac{-i}{a_{2}} \ell\left[\rho_{k-1}\left(\check{Z}_{j_{h}}(\ell), \ell\right), Z_{i_{k}}(\ell)\right] X\right) \\
& =\frac{1}{2} \frac{c_{1} c_{2}}{i a_{2}}\left(\frac{i}{a_{1}} \bar{X}+\frac{-i}{a_{2}} X\right) \\
& =-\frac{c_{1} c_{2}}{a_{2}} \frac{i}{a_{1} a_{2}} \frac{1}{2 i}\left(a_{1} X-a_{2} \bar{X}\right) \\
& =C(\ell) \check{Z}_{j_{h}}(\ell),
\end{aligned}
$$

where $C(\ell)=-i c_{1} c_{2} / a_{1} a_{2}^{2}$. This completes the proof of statement (2). 
The verification of statement (3) just amounts to an inspection of Case 1 in the definition of $Z_{i_{k}}(\ell)$ above. Turning to statement (4), we see that the equation $\ell\left(Z_{j_{k}}(\ell)\right)=0$ is equivalent to the equation

$$
\ell\left[\bar{Z}_{j_{k}}, V_{k}(\ell)\right] \ell_{j_{k}}+\ell\left[Z_{j_{k}}(\ell), V_{k}(\ell)\right] \bar{\ell}_{j_{k}}=0
$$

and that by definition of the function $V_{k}(\ell)$, this is in turn equivalent to

$$
\left(\beta_{1, k}(\ell) \overline{\beta_{1, k}(\ell)}+\beta_{2, k}(\ell) \overline{\beta_{2, k}(\ell)}\right) \ell_{j_{k}}+\left(\beta_{1, k}(\ell)^{2}+\beta_{2, k}(\ell)^{2}\right) \bar{\ell}_{j_{k}}=0 .
$$

Finally, we find that this last equation is equivalent to the system

$$
\begin{aligned}
& \overline{\beta_{1, k}(\ell)} \ell_{j_{k}}+\beta_{1, k}(\ell) \bar{\ell}_{j_{k}}=0, \\
& \overline{\beta_{2, k}(\ell)} \ell_{j_{k}}+\beta_{2, k}(\ell) \bar{\ell}_{j_{k}}=0,
\end{aligned}
$$

and the result follows.

In the preceding proof a fact emerged which we shall need later.

Corollary 4.3. Let $j=j_{k}$ be a value of the sequence $\mathbf{j}$. Then $U_{k}(\ell)=Z_{j_{k}}(\ell)$ modulo $\mathfrak{s}_{j^{\prime}}$.

Proof. Recall that $U_{k}(\ell)=\rho_{k-1}\left(Z_{j_{k}}(\ell), \ell\right)$, and so the only case where this is not clear from the properties of $\rho_{k-1}$ is the case $j^{\prime \prime}-j^{\prime}=2$, and $\left\{j^{\prime}+1, j^{\prime \prime}\right\}=\left\{j_{h}, j_{k}\right\}$ with $h<k$. But in this case we saw in part (2) of the preceding proof that $Z_{j_{k}}(\ell)$ is a multiple of $\check{Z}_{j_{h}}(\ell)$. Since $\ell\left[\check{Z}_{j_{h}}(\ell), V_{h}(\ell)\right]=0$ by construction of $\check{Z}_{j_{h}}(\ell)$, we have $\ell\left[Z_{j_{k}}(\ell), V_{h}(\ell)\right]=0$, and so the coefficient of $U_{h}(\ell)$ in the expansion of $\rho_{k-1}\left(Z_{j_{k}}(\ell), \ell\right)$ vanishes.

Observe that the preceding corollary gives a new cross-section for coadjoint orbits even in the nilpotent case. Thus we make the following definition.

Definition 5 (Adaptable cross-section). Let $\Omega$ be a layer belonging to the ultrafine stratification resulting from a choice of adaptable basis. The resulting cross-section $\Sigma \subset \Omega$ for coadjoint orbits in $\Omega$ will be called the adaptable cross-section.

Example 4.1. As in Examples 1.1 and 2.1. let $\mathfrak{g}=\operatorname{span}\left\{Z_{1}, Z_{2}, Y, X_{1}, X_{2}, A\right\}$, where $\left[X_{j}, Y\right]=Z_{j},\left[A, Z_{1}+i Z_{2}\right]=(1+i)\left(Z_{1}+i Z_{2}\right)$, and $\left[A, X_{1}+i X_{2}\right]=$ $(1+i)\left(X_{1}+i X_{2}\right)$. Choose the adaptable basis $\left(Z_{1}+i Z_{2}, Z_{1}-i Z_{2}, Y, X_{1}+i X_{2}, X_{1}-\right.$ $\left.i X_{2}, A\right)$ of $\mathfrak{s}$. We get the following ultrafine layers and cross-sections, listed in order:

1. $\mathbf{e}=\{1,3,4,6\}, \mathbf{j}=(6,4)$. Then $\varphi=\{1\}$ and

$$
\begin{aligned}
\Omega_{\mathbf{e}, \mathbf{j}, \varphi} & =\Omega_{\mathbf{e}, \mathbf{j}}=\{\ell=(z, \bar{z}, y, x, \bar{x}, a) \mid z \neq 0\}, \\
\Sigma_{\mathbf{e}, \mathbf{j}, \varphi} & =\{\ell=(z, \bar{z}, y, x, \bar{x}, a)|| z \mid=1, a=y=0, \mathfrak{R e}(\bar{z} x)=0\} \\
& =\left\{\ell=\left(e^{i \theta}, e^{-i \theta}, 0, i \xi e^{-i \theta},-i \xi e^{i \theta}, 0\right), \theta, \xi \in \mathbb{R}\right\} .
\end{aligned}
$$

2. $\mathbf{e}=\{4,6\}, \mathbf{j}=(6)$. Then $\varphi=\{4\}$ and

$$
\begin{aligned}
& \Omega_{\mathbf{e}, \mathbf{j}, \varphi}=\Omega_{\mathbf{e}, \mathbf{j}}=\left\{\ell=\left(0,0, y, x_{1}+i x_{2}, x_{1}-i x_{2}, a\right) \mid x_{1}+i x_{2} \neq 0\right\}, \\
& \Sigma_{\mathbf{e}, \mathbf{j}, \varphi}=\left\{\ell \in \Omega_{\mathbf{e}, \mathbf{j}, \varphi}|a=0,| x \mid=1\right\}=\left\{\ell=\left(0,0, \eta, e^{i \theta}, e^{-i \theta}, 0\right), \eta, \theta \in \mathbb{R}\right\} .
\end{aligned}
$$

3. $\mathbf{e}=\emptyset, \mathbf{j}=\emptyset$. Then $\varphi=\emptyset$ and

$$
\Sigma_{\mathbf{e}, \mathbf{j}, \varphi}=\Omega_{\mathbf{e}, \mathbf{j}, \varphi}=\{\ell=(0,0, \eta, 0,0, \alpha), \eta, \alpha \in \mathbb{R}\} .
$$


Example 4.2. As in Example 1.2 and 2.2 let $\mathfrak{g}$ be the Lie algebra with the adaptable basis $\{Z, \bar{Z}, Y, \bar{Y}, X, \bar{X}, A\}$ and relations:

$$
\begin{aligned}
& {[X, Y]=0,[X, \bar{Y}]=(1-i) \bar{Z},} \\
& {[A, X]=(1+i) X,[A, Y]=(1+2 i) Y,[A, Z]=(2+i) Z .}
\end{aligned}
$$

We get the following ultrafine layers and cross-sections:

1. $\mathbf{e}=\{1,3,4,5,6,7\}, \mathbf{j}=(7,6,5)$. Then $\varphi=\{1\}$ and

$$
\begin{aligned}
\Omega_{\mathbf{e}, \mathbf{j}, \varphi} & =\{\ell=(z, \bar{z}, y, \bar{y}, x, \bar{x}, a) \mid z \neq 0\}, \\
\Sigma_{\mathbf{e}, \mathbf{j}, \varphi} & =\{\ell=(z, \bar{z}, y, \bar{y}, x, \bar{x}, a)|| z \mid=1, a=0, x=y=0\} \\
& =\left\{\ell=\left(e^{i \theta}, e^{-i \theta}, 0,0,0,0,0\right), \theta \in \mathbb{R}\right\} .
\end{aligned}
$$

2. $\mathbf{e}=\{3,7\}, \mathbf{j}=(7)$. Then $\varphi=\{3\}$ and

$$
\begin{aligned}
\Omega_{\mathbf{e}, \mathbf{j}, \varphi} & =\{\ell=(0,0, y, \bar{y}, x, \bar{x}, a) \mid y \neq 0\}, \\
\Sigma_{\mathbf{e}, \mathbf{j}, \varphi} & =\{\ell=(0,0, y, \bar{y}, x, \bar{x}, a)|| y \mid=1, a=0\} \\
& =\left\{\ell=\left(0,0, e^{i \theta}, e^{-i \theta}, \xi, \bar{\xi}, 0\right), \theta \in \mathbb{R}, \xi \in \mathbb{C}\right\} .
\end{aligned}
$$

3. $\mathbf{e}=\{5,7\}, \mathbf{j}=(7)$. Then $\varphi=\{5\}$ and

$$
\begin{aligned}
\Omega_{\mathbf{e}, \mathbf{j}, \varphi} & =\{\ell=(0,0,0,0, x, \bar{x}, a) \mid x \neq 0\}, \\
\Sigma_{\mathbf{e}, \mathbf{j}, \varphi} & =\{\ell=(0,0,0,0, x, \bar{x}, a)|| x \mid=1, a=0\} \\
& =\left\{\ell=\left(0,0,0,0, e^{i \theta}, e^{-i \theta}, 0\right), \theta \in \mathbb{R}\right\} .
\end{aligned}
$$

4. $\mathbf{e}=\emptyset, \mathbf{j}=\emptyset, \varphi=\emptyset$ and

$$
\Sigma_{\mathbf{e}, \mathbf{j}, \varphi}=\Omega_{\mathbf{e}, \mathbf{j}, \varphi}=\{\ell=(0,0,0,0,0,0, \alpha), \alpha \in \mathbb{R}\} .
$$

Example 4.3. As in Examples 1.3 and 2.3, let $\mathfrak{g}$ be the Lie algebra with the adaptable basis $\{Z, X+i Y, X-i Y, A\}$ and relations

$$
[X, Y]=Z,[A, X+i Y]=(1+i)(X+i Y),[A, Z]=2 Z .
$$

1. $\mathbf{e}=\{1,2,3,4\}, \mathbf{j}=(3,4)$. Then $\varphi=\{1\}$ and

$$
\begin{aligned}
\Omega_{\mathbf{e}, \mathbf{j}, \varphi} & =\{\ell=(z, x+i y, x-i y, a) \mid z \neq 0\}, \\
\Sigma_{\mathbf{e}, \mathbf{j}, \varphi} & =\{\ell=(z, x+i y, x-i y, a)|| z \mid=1, a=x=y=0\} \\
& =\{\ell=(\epsilon, 0,0,0), \epsilon= \pm 1\} .
\end{aligned}
$$

2. $\mathbf{e}=\{2,4\}, \mathbf{j}=(4)$. Then $\varphi=\{2\}$ and

$$
\begin{aligned}
\Omega_{\mathbf{e}, \mathbf{j}, \varphi} & =\{\ell=(0, x+i y, x-i y, a) \mid x+i y \neq 0\}, \\
\Sigma_{\mathbf{e}, \mathbf{j}, \varphi} & =\{\ell=(0, x+i y, x-i y, a)|| x+i y \mid=1, a=0\} \\
& =\left\{\ell=\left(0, e^{i \theta}, e^{-i \theta}, 0\right), \theta \in \mathbb{R}\right\} .
\end{aligned}
$$

3. $\mathbf{e}=\emptyset, \mathbf{j}=\emptyset, \varphi=\emptyset$ and

$$
\Sigma_{\mathbf{e}, \mathbf{j}, \varphi}=\Omega_{\mathbf{e}, \mathbf{j}, \varphi}=\{\ell=(0,0,0, \alpha), \alpha \in \mathbb{R}\} .
$$

Let $\Omega$ be an ultrafine layer and let $\sigma: \Omega \rightarrow \Sigma$ be the natural $G$-invariant map. Though $\Omega$ is defined explicitly, it is not necessarily a submanifold of $\mathfrak{g}^{*}$ (unless it is the minimal, Zariski open layer). Nevertheless it is clear that many of the functions defined so far on $\Omega$ (for example the rational functions) are in fact analytic at each point $\ell \in \Omega$. To be precise, we make the following definition. 
Definition 6 (Analytic functions). Let $O$ be an open subset of $\Omega, E$ an analytic manifold, and $f: O \rightarrow E$. We shall say that $f$ is analytic at $\ell \in O$ if there is an open subset $\mathcal{V}$ of $\mathfrak{g}^{*}$ containing $\ell$ and an analytic function $\tilde{f}_{\ell}: \mathcal{V} \rightarrow E$ such that $\left.\tilde{f}\right|_{\mathcal{V} \cap O}=\left.f\right|_{\mathcal{V} \cap O}$. If $f$ is analytic at every $\ell \in O$, we say that $f$ is analytic on $O$.

We denote by $\mathcal{A}(O, E)$ the algebra of all $E$-valued analytic functions on $O$, and if $E=\mathbb{C}$, then we just write $\mathcal{A}(O)=\mathcal{A}(O, E)$.

We claim that $\sigma \in \mathcal{A}\left(\Omega, \mathfrak{g}^{*}\right)$. To see this, we take a closer look at the parametrization of each orbit in $\Omega$, following the adaptation of [8, Proposition 5] with a precise choice of supplementary basis as described in [5, proof of Proposition 2.6] and 4, Lemma 1.3.12, Proposition 1.3.13].

Let $\mathcal{U}$ be the Zariski open subset of $\mathfrak{g}^{*}$ containing $\Omega$ on which each of the rational functions $\rho_{k}(Z, \ell), \mathbf{b}_{i_{k}}(\ell), U_{k}(\ell), V_{k}(\ell), \ell\left[Z_{j_{k}}, V_{k}(\ell)\right]$ and $\ell\left[Z_{i_{k}}, U_{k}(\ell)\right], 1 \leq k \leq d$, are non-singular. For each $1 \leq k \leq d$ and for $\epsilon_{k}=1$ or 2 , the rational function $\beta_{k, \epsilon_{k}}(\ell)$ is non-singular on $\mathcal{U}$. For each $\epsilon \in\{1,2\}^{d}$, set

$$
\mathcal{U}_{\epsilon}=\left\{\ell \in \mathcal{U} \mid \beta_{k, \epsilon_{k}}(\ell) \neq 0,1 \leq k \leq d\right\} .
$$

Note that $\mathcal{U}_{\epsilon} \cap \Omega=O_{\epsilon} \in F$. For each $j \in \mathbf{e}$, the functions $\phi_{j}^{\epsilon}$ as defined in Section 3 are in fact defined on all of $\mathcal{U}_{\epsilon}$. Set

$$
X_{k}^{\epsilon}(\ell)=\mathfrak{R e}\left(\phi_{j_{k}}^{\epsilon}(\ell)^{-1} U_{k}(\ell)\right), \quad Y_{k}^{\epsilon}(\ell)=\mathfrak{R e}\left(\phi_{i_{k}}^{\epsilon}(\ell)^{-1} V_{k}(\ell)\right) .
$$

Next, for each $1 \leq a \leq 2 d$, set

$$
r_{a}^{\epsilon}(\ell)=\frac{X_{k}^{\epsilon}(\ell)}{\left|\ell\left[Z_{i_{k}}, X_{k}^{\epsilon}(\ell)\right]\right|}
$$

if $e_{a}=i_{k}$, while if $e_{a}=j_{k}$, set

$$
r_{a}^{\epsilon}(\ell)=\frac{Y_{k}^{\epsilon}(\ell)}{\left|\ell\left[Z_{j_{k}}, Y_{k}^{\epsilon}(\ell)\right]\right|} .
$$

If $j=e_{a} \in \mathbf{e}$ with $j-1 \in I$, then one can write

$$
\operatorname{ad}^{*} r_{a}^{\epsilon}(\ell) \ell=\zeta_{a}^{\epsilon}(\ell) Z_{j}^{*} \bmod \mathfrak{s}_{j}^{\perp} .
$$

This relation also holds if $j \in I$, while if $j \notin I$, then

$$
\operatorname{ad}^{*} r_{a}^{\epsilon}(\ell) \ell=\zeta_{a}^{\epsilon}(\ell) Z_{j}^{*}+\overline{\zeta_{a}^{\epsilon}(\ell)} Z_{j+1}^{*} \bmod \mathfrak{s}_{j+1}^{\perp} .
$$

Since in this case $Z_{j+1}=\bar{Z}_{j}$, the function $\zeta_{a}^{\epsilon}(\ell)$ is $\mathbb{T}$-valued. If also $j+1=e_{a+1} \in \mathbf{e}$, then similarly

$$
\operatorname{ad}^{*} r_{a+1}^{\epsilon}(\ell) \ell=\zeta_{a+1}^{\epsilon}(\ell) Z_{j}^{*}+\overline{\zeta_{a+1}^{\epsilon}(\ell)} Z_{j+1}^{*} \bmod \mathfrak{s}_{j+1}^{\perp} .
$$

The following important property of the functions $r_{a}^{\epsilon}$ is an immediate consequence of Proposition 4.1] (See also [5, Propositions 1.5 and 1.8].)

Corollary 4.4. Let $\ell$ belong to the fine layer $\Omega_{\mathbf{e}, \mathbf{j}}$, and let $j=e_{a} \in \mathbf{e}$. Then for any covering set $O_{\epsilon}$ containing $\ell$, we have $j \in \varphi(\ell)$ if and only if $\gamma_{j}\left(r_{a}^{\epsilon}(\ell)\right) \neq 0$.

Note that the functions $\phi_{j}^{\epsilon}, r_{a}^{\epsilon}$, and $\zeta_{a}^{\epsilon}$ are analytic at each point of $\mathcal{U}_{\epsilon}$. Finally, let $Q^{\epsilon}: \mathbb{R}^{2 d} \times \mathcal{U}_{\epsilon} \rightarrow \mathfrak{g}^{*}$ be defined by

$$
Q^{\epsilon}(t, \ell)=\exp \left(t_{1} r_{1}^{\epsilon}(\ell)\right) \exp \left(t_{2} r_{2}^{\epsilon}(\ell)\right) \cdots \exp \left(t_{2 d} r_{2 d}^{\epsilon}(\ell)\right) \ell .
$$

Now suppose that $\ell$ belongs to the ultrafine layer $\Omega$. The fact that $t \mapsto Q^{\epsilon}(t, \ell)$ is a bijection between $\mathbb{R}^{2 d}$ and $\mathcal{O}_{\ell}$ of the form described in [8, Proposition 5] is 
fundamental to the work of 5 . Since our work here includes a slight generalization of [5], we recall the argument for this fact.

Following [8, page 92], let $\mathfrak{k}$ be a subalgebra of $\mathfrak{g}$, and let $\left(X_{1}, X_{2}, \ldots, X_{a}\right)$ be an ordered basis of $\mathfrak{g}$ modulo $\mathfrak{k}$. We say that $\left(X_{1}, X_{2}, \ldots, X_{a}\right)$ is supplementary for $\mathfrak{k}$ if

$$
\left(t_{1}, t_{2}, \ldots, t_{a}\right) \mapsto \exp \left(t_{1} X_{1}\right) \exp \left(t_{2} X_{2}\right) \cdots \exp \left(t_{a} X_{a}\right) K
$$

is a diffeomorphism of $\mathbb{R}^{a}$ onto $G / K$.

Lemma 4.4. Let $\ell \in \Omega \cap \mathcal{U}_{\epsilon}$, and let $m \in$ I. If $\mathfrak{g}_{m}^{\ell} \neq \mathfrak{s}$, then $\left(r_{1}^{\epsilon}(\ell), r_{2}^{\epsilon}(\ell), \ldots, r_{a}^{\epsilon}(\ell)\right)$ is a basis of $\mathfrak{g}$ modulo $\mathfrak{g} \cap \mathfrak{g}_{m}^{\ell}$ supplementary for $\mathfrak{g} \cap \mathfrak{g}_{m}^{\ell}$, where

$$
a=\max \left\{1 \leq b \leq 2 d \mid e_{b} \leq m\right\} .
$$

Proof. We proceed by induction on $m$; if $m=0$, then there is nothing to prove. Suppose that $m>0$, and suppose that the result is true for all indices $i \in I, i<m$. Assume that $\mathfrak{g}_{m}^{\ell} \neq \mathfrak{s}$. By induction the result holds for $m^{\prime}$, so that if $\mathfrak{g}_{m^{\prime}}^{\ell} \neq \mathfrak{s}$, then $\left(r_{1}^{\epsilon}(\ell), r_{2}^{\epsilon}(\ell), \ldots, r_{a^{\prime}}^{\epsilon}(\ell)\right)$ is a basis of $\mathfrak{g}$ modulo $\mathfrak{g} \cap \mathfrak{g}_{m^{\prime}}^{\ell}$ supplementary for $\mathfrak{g} \cap \mathfrak{g}_{m^{\prime}}^{\ell}$, where $a^{\prime}=\max \left\{1 \leq b \leq 2 d \mid e_{b} \leq m^{\prime}\right\}\left(a^{\prime}\right.$ can be 0$)$. Now $\mathfrak{g}_{m^{\prime}}^{\ell}=\mathfrak{g}_{m}^{\ell}$ if and only if $a^{\prime}=a$, so if this is the case, then we are done. Suppose then that $\mathfrak{g}_{m^{\prime}}^{\ell} \neq \mathfrak{g}_{m}^{\ell}$, and set $d_{a}=a-a^{\prime}=\operatorname{dim}\left(\mathfrak{g}_{m^{\prime}}^{\ell} / \mathfrak{g}_{m}^{\ell}\right)$. It is now clear that it remains for us to show that $r_{a}^{\epsilon}(\ell)$ (resp. $r_{a-1}^{\epsilon}(\ell), r_{a}^{\epsilon}(\ell)$ ) is a supplementary basis for $\mathfrak{g}_{m^{\prime}}^{\ell}$ modulo $\mathfrak{g}_{m}^{\ell}$ if $d_{a}=1$ (resp. $d_{a}=2$ ). Set $j=m^{\prime}+1$, so that we have $j \in \mathbf{e}$.

From [8, Lemma 5 and proof of Proposition 5, part II], we glean the following. If $j \in \varphi$, then $\mathfrak{g}_{j^{\prime \prime}}^{\ell}$ is an ideal of codimension one in $\mathfrak{g}_{j^{\prime}}^{\ell}$ (in [8, proof of Proposition 5 , part II], this occurs in cases $a_{1}$ and $b_{1}$, as well as in cases $a_{22}$ and $b_{23}$ when $\gamma_{j} \neq 0$ on $\mathfrak{g}_{j^{\prime}}^{\ell}$ ). Hence a supplementary basis is obtained by choosing any element $A \in \mathfrak{g}_{j^{\prime}}^{\ell} \cap \mathfrak{g}$ for which $\gamma_{j}(A) \neq 0$. Corollary 4.4 shows immediately that $r_{a}^{\epsilon}(\ell)$ is such an element.

On the other hand if $j \notin \varphi$, then a supplementary basis is obtained by taking independent elements belonging to $\mathfrak{g}_{j^{\prime}}^{\ell} \cap C_{j}$, so that their image under $\eta_{j}$ lies in $\mathfrak{a}(\ell)$ (this occurs in cases $a_{21}, b_{21}$, and $b_{22}$, as well as in cases $a_{22}$ and $b_{23}$ when $\gamma_{j}=0$ on $\mathfrak{g}_{j^{\prime}}^{\ell}$ of loc. cit.). Again Corollary 4.4 shows immediately that if $d_{a}=1$, then $r_{a}^{\epsilon}(\ell)$, or if $d_{a}=2$, then $\left\{r_{a-1}^{\epsilon}(\ell), r_{a}^{\epsilon}(\ell)\right\}$ are such elements.

Applying the preceding lemma to the case where $m=n$, we get

Corollary 4.5. For each $\ell$ belonging to the fine layer with $O_{\epsilon}$ a covering set containing $\ell$, the mapping $t \mapsto Q^{\epsilon}(t, \ell)$ is a diffeomorphism between $\mathbb{R}^{2 d}$ and $\mathcal{O}_{\ell}$.

The application of [8, Proposition 5] to the above supplementary basis produces the following description of $Q^{\epsilon}$.

Proposition 4.6 (4, Proposition 1.3.6, Proposition 1.3.13]). Let $\ell$ belong to the fine layer $\Omega_{\mathbf{e}, \mathbf{j}}$ with $O_{\epsilon}$ any covering set containing $\ell$. Then $t \mapsto Q^{\epsilon}(t, \ell)$ is a real analytic function in the variables $t_{1}, t_{2}, \ldots, t_{2 d}$ and has the following form.

Let $1 \leq j \leq n$ such that $j-1 \in I$, and let $a=\min \left\{1 \leq b \leq 2 d \mid e_{b} \geq j\right\}$. Set $\mu_{j}^{\epsilon}(t, \ell)=\mu_{j}\left(\exp \left(t_{1} r_{1}^{\epsilon}(\ell)\right) \cdots \exp \left(t_{a-1} r_{a-1}^{\epsilon}(\ell)\right)\right)$.

If $j \notin \mathbf{e}$, then

$$
Q_{j}^{\epsilon}(t, \ell)=\mu_{j}^{\epsilon}(t, \ell) \ell_{j}+Y_{j}^{\epsilon}(t, \ell) .
$$

Suppose that $j \in \mathbf{e}$. If $j \notin \varphi(\ell)$, then

$$
Q_{j}^{\epsilon}(t, \ell)=\mu_{j}^{\epsilon}(t, \ell)\left(\ell_{j}+t_{a} \zeta_{a}^{\epsilon}(\ell)\right)+Y_{j}^{\epsilon}(t, \ell)
$$


if $\left\{j, j^{\prime \prime}\right\}$ contains only one index belonging to $\mathbf{e}$, while

$$
Q_{j}^{\epsilon}(t, \ell)=\mu_{j}^{\epsilon}(t, \ell)\left(\ell_{j}+t_{a} \zeta_{a}^{\epsilon}(\ell)+t_{a+1} \zeta_{a+1}^{\epsilon}(\ell)\right)+Y_{j}^{\epsilon}(t, \ell)
$$

if $j$ and $j^{\prime \prime}=j+1$ both belong to e. Finally, if $j \in \varphi(\ell)$, then

$$
Q_{j}^{\epsilon}(t, \ell)=\mu_{j}^{\epsilon}(t, \ell)\left(e^{t_{a} \gamma_{j}\left(r_{a}^{\epsilon}(\ell)\right)} \mathbf{b}_{j}(\ell)^{-1}\right)+Y_{j}^{\epsilon}(t, \ell) .
$$

In each of the cases above, $Y_{j}^{\epsilon}(t, \ell)$ depends only upon $t_{1}, t_{2}, \ldots, t_{a-1}$ and $\ell_{1}, \ell_{2}$, $\ldots, \ell_{j}$.

Each of the functions $\zeta_{a}^{\epsilon}(\ell)$ (or $\zeta_{a}^{\epsilon}(\ell)$ and $\left.\zeta_{a+1}^{\epsilon}(\ell)\right), \mu_{j}^{\epsilon}(t, \ell), \gamma_{j}\left(r_{a}^{\epsilon}(\ell)\right)$, and $\mathbf{b}_{j}(\ell)^{-1}-\ell_{j}$ depend only upon $\ell_{1}, \ell_{2}, \ldots, \ell_{j-1}$.

The functions $Y_{j}^{\epsilon}(t, \ell)$ depend only upon $\ell_{1}, \ell_{2}, \ldots, \ell_{j-1}$ unless $j=j_{k}$ and $j+1$ is in $\mathbf{e}$.

A proof for all these facts about dependency can be found in [4, Proposition 1.3.6, Lemma 1.3.7, Lemma 1.3.10, Lemma 1.3.12] and [5, Lemma 2.1]. Now we are ready to show that $\sigma$ is analytic.

Theorem 4.7 (The cross-section mapping is regular). Let $\mathfrak{g}$ be an exponential Lie algebra, choose an adaptable basis for its complexification, and let $\Omega=\Omega_{\mathbf{e}, \mathbf{j}, \varphi}$ be a corresponding ultrafine layer in $\mathfrak{g}^{*}$ with cross-section $\Sigma$. Then the cross-section mapping $\sigma: \Omega \rightarrow \Sigma$ is analytic on $\Omega$.

Proof. Fix $g \in \Omega$ and choose $\epsilon \in\{1,2\}^{d}$ such that $g \in \mathcal{U}_{\epsilon}$. We have a unique $s=t^{\epsilon}(g) \in \mathbb{R}^{2 d}$ such that $Q^{\epsilon}(s, g)=\sigma(g)$. Fix $\epsilon^{\prime}$ such that $\sigma(g) \in \mathcal{U}_{\epsilon^{\prime}}$. Define $f^{\epsilon^{\prime}}: \mathcal{U}_{\epsilon^{\prime}} \rightarrow \mathbb{R}^{2 d}$ componentwise for $1 \leq a \leq 2 d$ by

$$
f_{a}^{\epsilon^{\prime}}(\ell)= \begin{cases}\mathfrak{R e}\left(\phi_{k}^{\epsilon^{\prime}}(\ell)^{-1} \ell\left(Z_{j}(\ell)\right)\right), & \text { if } j=e_{a} \notin \varphi, j \in\left\{i_{k}, j_{k}\right\} \\ \left|b_{j}(\ell)\right|^{-2}-1, & \text { if } j \in \varphi\end{cases}
$$

Observe that $f^{\epsilon^{\prime}}(\sigma(g))=0$ and that for $\ell \in \mathcal{U}_{\epsilon^{\prime}} \cap \Omega, f^{\epsilon^{\prime}}(\ell)=0$ if and only if $\ell \in \Sigma$.

Set $\mathcal{W}=\left(Q^{\epsilon}\right)^{-1}\left(\mathcal{U}_{\epsilon^{\prime}}\right) \subset \mathbb{R}^{2 d} \times \mathcal{U}_{\epsilon}$ and define $F: \mathcal{W} \rightarrow \mathbb{R}^{2 d}$ by

$$
F(t, \ell)=f^{\epsilon^{\prime}}\left(Q^{\epsilon}(t, \ell)\right)
$$

It is clear that $F(t, \ell)$ is analytic at each $(t, \ell) \in \mathcal{W}$. We put

$$
J(s, g)=\left[\frac{\partial F_{a}(s, g)}{\partial t_{b}}\right]_{1 \leq a, b \leq 2 d} .
$$

We claim that $\operatorname{det}(J(s, g)) \neq 0$. Assuming that this claim is true, we apply the Implicit Function Theorem to obtain a neighborhood $\mathcal{V}(g)$ of $g$ and an analytic function $t^{\epsilon}: \mathcal{V}(g) \rightarrow \mathbb{R}^{2 d}$ for which both $\left\{\left(t^{\epsilon}(\ell), \ell\right) \mid \ell \in \mathcal{V}(g)\right\} \subset \mathcal{W}$ and $F\left(t^{\epsilon}(\ell), \ell\right)=$ 0 , for all $\ell \in \mathcal{V}(g)$, hold. We then set

$$
\tilde{\sigma}(\ell)=Q^{\epsilon}\left(t^{\epsilon}(\ell), \ell\right)
$$

Then $\tilde{\sigma}$ is analytic at each point of $\mathcal{V}(g)$, and for each $\ell \in \mathcal{V}(g) \cap \Omega$ we have that $\tilde{\sigma}(\ell)$ belongs to the coadjoint orbit of $\ell$; hence it belongs to $\Omega$. But

$$
0=F\left(t^{\epsilon}(\ell), \ell\right)=f(\tilde{\sigma}(\ell)),
$$

so that from the observation above, $\tilde{\sigma}(\ell) \in \Sigma$. Thus the proof will be complete once we have verified the claim above.

To this end, we examine the coordinate functions $F_{a}$. For each $j \in \mathbf{e}$ write $Z_{j}(\ell)=b_{1, j}(\ell) Z_{j}+b_{2, j}(\ell) \bar{Z}_{j}$ and set $\ell^{\prime}=\ell^{\prime}(t, g)=\pi_{j^{\prime}}\left(Q^{\epsilon}(t, g)\right)$. Now fix $a, 1 \leq$ $a \leq 2 d, j=e_{a}$. Fix $k, 1 \leq k \leq d$, such that $j \in\left\{i_{k}, j_{k}\right\}$. Put $d_{j}=\operatorname{Card}\left(\mathbf{e} \cap\left\{j, j^{\prime \prime}\right\}\right)$. 
Case 1. Suppose that $d_{j}=1$ and that $j \notin \varphi$. In this case from [5, Lemma 2.4], we have that $\zeta_{a}^{\epsilon}(u \ell)= \pm \operatorname{sign}\left(\mu_{j}(u)\right) \zeta_{a}^{\epsilon}(\ell), u \in G, \ell \in \Omega$. It follows that for each $t$ in a neighborhood of $s$, there is a choice of sign $\delta= \pm 1$ such that

$$
F_{a}(t, g)=\delta \phi_{k}^{\epsilon^{\prime}}\left(\ell^{\prime}\right)^{-1}\left|\mu_{j}(t, g)\right|\left(b_{1, j}\left(\ell^{\prime}\right) \zeta_{a}^{\epsilon}\left(\ell^{\prime}\right)+b_{2, j}\left(\ell^{\prime}\right) \overline{\zeta_{a}^{\epsilon}}\left(\ell^{\prime}\right)\right) t_{a} \bmod t_{1}, t_{2}, \ldots, t_{a-1} .
$$

Hence $\partial F_{a}(s, g) / \partial t_{b}=0$ for $b>a$ and

$$
\left|\frac{\partial F_{a}(s, g)}{\partial t_{a}}\right|=\left|\mu_{j}(s, g)\right|\left(b_{1, j}\left(\ell^{\prime}(s, g)\right) \zeta_{a}^{\epsilon}\left(\ell^{\prime}(s, g)\right)+b_{2, j}\left(\ell^{\prime}(s, g)\right) \overline{\zeta_{a}^{\epsilon}}\left(\ell^{\prime}(s, g)\right)\right) .
$$

Now consider the quantity $b_{1, j}\left(\ell^{\prime}\right) \zeta_{a}^{\epsilon}\left(\ell^{\prime}\right)+b_{2, j}\left(\ell^{\prime}\right) \overline{\zeta_{a}^{\epsilon}}\left(\ell^{\prime}\right)$. An elementary computation shows that if $j=i_{k}$, then

$$
b_{1, j}\left(\ell^{\prime}\right) \zeta_{a}^{\epsilon}\left(\ell^{\prime}\right)+b_{2, j}\left(\ell^{\prime}\right) \overline{\zeta_{a}^{\epsilon}}\left(\ell^{\prime}\right)=\frac{\phi_{k}^{\epsilon^{\prime}}\left(\ell^{\prime}\right)^{-1} \ell^{\prime}\left[V_{k}\left(\ell^{\prime}\right), U_{k}\left(\ell^{\prime}\right)\right]}{\left|\ell^{\prime}\left[Z_{j}, U_{k}\left(\ell^{\prime}\right)\right]\right|},
$$

while if $j=j_{k}$, then

$$
b_{1, j}\left(\ell^{\prime}\right) \zeta_{a}^{\epsilon}\left(\ell^{\prime}\right)+b_{2, j}\left(\ell^{\prime}\right) \overline{\zeta_{a}^{\epsilon}}\left(\ell^{\prime}\right)=\frac{\phi_{k}^{\epsilon^{\prime}}\left(\ell^{\prime}\right)^{-1} \ell^{\prime}\left[U_{k}\left(\ell^{\prime}\right), V_{k}\left(\ell^{\prime}\right)\right]}{\left|\ell^{\prime}\left[Z_{j}, V_{k}\left(\ell^{\prime}\right)\right]\right|} .
$$

In either case we see that $\partial F_{a}(s, g) / \partial t_{a} \neq 0$.

Case 2. Suppose that $d_{j}=2$. Here $j+1=e_{a+1} \in \mathbf{e}$, and by part (a) of Lemma 4.2. $j \notin \varphi$. Then modulo the variables $t_{1}, t_{2}, \ldots, t_{a-1}$, we have

$$
\begin{aligned}
F_{a}(t, g)=\phi_{h}^{\epsilon^{\prime}}\left(\ell^{\prime}\right)^{-1}\{( & \left.b_{1, j}\left(\ell^{\prime}\right) \mu_{j}(t, g) \zeta_{a}^{\epsilon}(\ell)+b_{2, j}\left(\ell^{\prime}\right) \overline{\mu_{j}}(t, g) \overline{\zeta_{a}^{\epsilon}}(\ell)\right) t_{a} \\
& \left.+\left(b_{1, j}\left(\ell^{\prime}\right) \mu_{j}(t, g) \zeta_{a+1}^{\epsilon}(\ell)+b_{2, j}\left(\ell^{\prime}\right) \overline{\mu_{j}}(t, g) \overline{\zeta_{a+1}^{\epsilon}}(\ell)\right) t_{a+1}\right\}
\end{aligned}
$$

and

$$
\begin{aligned}
F_{a+1}(t, g)=\phi_{k}^{\epsilon^{\prime}} & \left(\ell^{\prime}\right)^{-1}\left\{\left(b_{1, j+1}\left(\ell^{\prime}\right) \mu_{j}(t, g) \zeta_{a}^{\epsilon}(\ell)+b_{2, j+1}\left(\ell^{\prime}\right) \overline{\mu_{j}}(t, g) \overline{\zeta_{a}^{\epsilon}}(\ell)\right) t_{a}\right. \\
& \left.+\left(b_{1, j+1}\left(\ell^{\prime}\right) \mu_{j}(t, g) \zeta_{a+1}^{\epsilon}(\ell)+b_{2, j+1}\left(\ell^{\prime}\right) \overline{\mu_{j}}(t, g) \overline{\zeta_{a+1}^{\epsilon}}(\ell)\right) t_{a+1}\right\} .
\end{aligned}
$$

As in Case $1, \partial F_{a} / \partial t_{b}=\partial F_{a+1} / \partial t_{b}=0$ for $b>a+1$. Set

$$
J_{a}(s, g)=\left[\begin{array}{cc}
\frac{\partial F_{a}(s, g)}{\partial t_{a}} & \frac{\partial F_{a}(s, g)}{\partial t_{a+1}} \\
\frac{\partial F_{a+1}(s, g)}{\partial t_{a}} & \frac{\partial F_{a+1}(s, g)}{\partial t_{a+1}}
\end{array}\right] .
$$

Then $J_{a}(s, g)$ is the product

$$
\left[\begin{array}{cc}
\phi_{h}^{\epsilon^{\prime}}\left(\ell^{\prime}\right)^{-1} & 0 \\
0 & \phi_{k}^{\epsilon^{\prime}}\left(\ell^{\prime}\right)^{-1}
\end{array}\right]\left[\begin{array}{ll}
b_{1, j}\left(\ell^{\prime}\right) & b_{2, j}\left(\ell^{\prime}\right) \\
b_{1, j}\left(\ell^{\prime}\right) & b_{2, j}\left(\ell^{\prime}\right)
\end{array}\right]\left[\begin{array}{cc}
\mu_{j}(s, g) & 0 \\
0 & \overline{\mu_{j}}(s, g)
\end{array}\right]\left[\begin{array}{cc}
\zeta_{a}^{\epsilon}(g) & \zeta_{a+1}^{\epsilon}(g) \\
\zeta_{a}^{\epsilon}(g) & \left.\frac{\zeta_{a+1}^{\epsilon}}{(g)}\right)
\end{array}\right],
$$

with $\ell^{\prime}=\ell^{\prime}(s, g)$. In [5. Proposition 1.5] it is shown that $\zeta_{a}^{\epsilon}(g)$ and $\zeta_{a+1}^{\epsilon}(g)$ are $\mathbb{R}$-linearly independent, and by an argument similar to part $(2)$ of the proof of Theorem 4.2, one finds that $b_{1, j}\left(\ell^{\prime}\right) b_{2, j+1}\left(\ell^{\prime}\right)-b_{2, j}\left(\ell^{\prime}\right) b_{1, j+1}\left(\ell^{\prime}\right) \neq 0$. It follows that $\operatorname{det} J_{a}(s, g) \neq 0$.

Case 3. Suppose that $j \in \varphi$. Here we use the semi-invariance of $\mathbf{b}_{j}$ :

$$
\begin{aligned}
F_{a}(t, g) & =\left|\mathbf{b}_{j}\left(Q^{\epsilon}(t, g)\right)\right|^{-2}-1 \\
& =\left|\mathbf{b}_{j}\left(\exp \left(t_{1} r_{1}^{\epsilon}(g)\right) \exp \left(t_{2} r_{2}^{\epsilon}(g)\right) \cdots \exp \left(t_{a} r_{a}^{\epsilon}(g) g\right)\right)\right|^{-2}-1 \\
& =\left|\mu_{j}(t, g)\right|^{2}\left|\mathbf{b}_{j}(g)\right|^{-2} \exp \left(2 \mathfrak{R e}\left(\gamma_{j}\left(r_{a}(g)\right)\right) t_{a}\right)-1
\end{aligned}
$$

from which we immediately see that $\partial F_{a}(s, g) / \partial t_{a} \neq 0$. 
It is now clear that

$$
\operatorname{det} J(s, g)=\prod_{a \mid d_{e_{a}}=1} \frac{\partial F_{a}(s, g)}{\partial t_{a}} \prod_{a \mid d_{e_{a}}=2} \operatorname{det} J_{a}(s, g) \neq 0 .
$$

Fix $\ell \in \Omega$; for any $\epsilon$ and $\epsilon^{\prime}$ with $\ell \in O_{\epsilon} \cap O_{\epsilon^{\prime}}$ and for any $j \in \mathbf{e}$, we have $\phi_{j}^{\epsilon^{\prime}}(\ell)= \pm \phi_{j}^{\epsilon}(\ell)$, by construction. It follows that $r_{a}^{\epsilon^{\prime}}(\ell)= \pm r_{a}^{\epsilon}(\ell)$. Set

$$
g^{\epsilon}(t, \ell)=\exp \left(t_{1} r_{1}^{\epsilon}(\ell)\right) \exp \left(t_{2} r_{2}^{\epsilon}(\ell)\right) \cdots \exp \left(t_{2 d} r_{2 d}^{\epsilon}(\ell)\right)
$$

so that $Q^{\epsilon}(t, \ell)=g^{\epsilon}(t, \ell) \ell$. The above shows that we have an analytic $\mathbb{R}^{2 d}$-valued function $t^{\epsilon}$ defined in a neighborhood of $\ell$ such that if $g^{\epsilon}(\ell):=g^{\epsilon}\left(t^{\epsilon}(\ell), \ell\right)$, then $g^{\epsilon}(\ell) \ell=\sigma(\ell)$. From Lemma 4.4 and the observation above we have $t^{\epsilon^{\prime}}(\ell)= \pm t^{\epsilon}(\ell)$, while $g^{\epsilon^{\prime}}(\ell)=g^{\epsilon}(\ell)$. Thus we have an explicit mapping $g: \Omega \rightarrow G$, independent of covering sets, such that $g(\ell) \ell=\sigma(\ell)$. Similarly if we set

$$
g^{a}(\ell)=\exp \left(t_{1} r_{1}^{\epsilon}(\ell)\right) \exp \left(t_{2} r_{2}^{\epsilon}(\ell)\right) \cdots \exp \left(t_{a} r_{a}^{\epsilon}(\ell)\right)
$$

then $g^{a}$ is independent of $\epsilon$ as well, and for any $m \in I$ we have

$$
\pi_{m}(\sigma(\ell))=g^{a}(\ell) \pi_{m}(\ell) \quad \text { with } \quad a=\max \left\{1 \leq b \leq 2 d \mid e_{b} \leq m\right\} .
$$

Corollary 4.8. The mappings $g^{a}, 1 \leq a \leq 2 d$, belong to $\mathcal{A}(\Omega, G)$.

\section{PROJECTION OF LAYERS AND CROSS-SECTIONS}

To adapt the method of 2 in the present context, we need to know the relationship between ultrafine layers and their cross-sections for the sequence of ideals $\left(\mathfrak{g}_{m}\right), m \in I$. In this section we shall describe the $\pi_{m}$-image of a cross-section for $G$-orbits as a subset of a $G_{m}$-cross-section.

Having, as always, our adaptable basis $\left\{Z_{1}, Z_{2}, \ldots, Z_{n}\right\}$ for $\mathfrak{s}$ in place, let $\Omega=$ $\Omega_{\mathbf{e}, \mathbf{j}, \varphi}$ be an ultrafine layer. Let $\ell \in \Omega$ and $m \in I$, and set $\ell^{m}=\pi_{m}(\ell)=$ $\left(\ell_{1}, \ell_{2}, \ldots, \ell_{m}\right)$. Now $\left\{Z_{1}, Z_{2}, \ldots, Z_{m}\right\}$ is an adaptable basis for $\mathfrak{s}_{m}$, so we may perform the layering procedure as described in Section 2 to obtain the jump set $\mathbf{e}\left(\ell^{m}\right)$, the index sequences $\mathbf{i}\left(\ell^{m}\right)$ and $\mathbf{j}\left(\ell^{m}\right)$ with polarizing subalgebras $\mathfrak{h}_{k}\left(\ell^{m}\right)$, and the dilation subset $\varphi\left(\ell^{m}\right) \subset \mathbf{e}\left(\ell^{m}\right)$. Put $K\left(\mathfrak{s}_{m}\right)=\left\{k \in K \mid j_{k} \leq m\right\}$ and write

$$
K\left(\mathfrak{s}_{m}\right)=\left\{k_{1}<k_{2}<\cdots<k_{d^{m}}\right\} .
$$

Let $\mathbf{i}^{m}, \mathbf{j}^{m}$ be the subsequences of $\mathbf{i}, \mathbf{j}$ resp., defined by $\mathbf{i}^{m}=\left(i_{k_{r}} \mid 1 \leq r \leq d^{m}\right)$ and $\mathbf{j}^{m}=\left(j_{k_{r}} \mid 1 \leq r \leq d^{m}\right)$. Finally put

$$
\mathbf{e}^{m}=\left\{1 \leq j \leq m \mid j \text { is a value of } \mathbf{i}^{m} \text { or } \mathbf{j}^{m}\right\} .
$$

Proposition 5.1. For each $\ell \in \Omega$ we have $\mathbf{i}\left(\ell^{m}\right)=\mathbf{i}^{m}, \mathbf{j}\left(\ell^{m}\right)=\mathbf{j}^{m}$, and $\mathbf{e}\left(\ell^{m}\right)=$ $\mathbf{e}^{m}$. Moreover, $\mathfrak{h}_{r}\left(\ell^{m}\right)=\mathfrak{h}_{k_{r}}(\ell) \cap \mathfrak{s}^{m}, 1 \leq r \leq d^{m}$, and if $r<d^{m}$ one has

$$
\mathfrak{h}_{r}\left(\ell^{m}\right)=\mathfrak{h}_{k}(\ell) \cap \mathfrak{s}_{m}, \quad k_{r}<k<k_{r+1} .
$$

Proof. Let $\ell \in \Omega$. We begin by showing that $\mathbf{i}\left(\ell^{m}\right)=\mathbf{i}^{m}$ and $\mathbf{j}\left(\ell^{m}\right)=\mathbf{j}^{m}$, by induction on the codimension $n-m$. Suppose first that $m=n-1$. Then the result follows from the proof of [1, Proposition 1.3].

Suppose that $m<n-1$. Consider first the case where $n^{\prime}=n-2$ and $n-1=$ $i_{d}(\ell)$. In this case we have $K\left(\mathfrak{s}_{n-2}\right)=\{1,2, \ldots, d-1\}$, and it is immediate from the definitions of $i_{k}\left(\ell^{n-2}\right)$ and $j_{k}\left(\ell^{n-2}\right)$ that $i_{k}\left(\ell^{n-2}\right)=i_{k}(\ell)$ and $j_{k}\left(\ell^{n-2}\right)=$ 
$j_{k}(\ell), 1 \leq k \leq d-1$. Now by induction we have $\mathbf{i}\left(\ell^{m}\right)=\mathbf{i}\left(\pi_{m}\left(\ell^{n-2}\right)\right)=\mathbf{i}^{m}$ and $\mathbf{j}\left(\ell^{m}\right)=\mathbf{j}\left(\pi_{m}\left(\ell^{n-2}\right)\right)=\mathbf{j}^{m}$.

Suppose then that we are not in the preceding situation. We observe the following: there is an adaptable basis $\left\{Z_{1}^{\prime}, Z_{2}^{\prime}, \ldots, Z_{n}^{\prime}\right\}$ for $\mathfrak{s}$ such that $\mathfrak{s}^{\circ}=\mathbb{C}$ $\operatorname{span}\left\{Z_{1}^{\prime}, Z_{2}^{\prime}, \ldots, Z_{n-1}^{\prime}\right\}$ is real, and such that the index sequences $\mathbf{i}^{\prime}(\ell), \mathbf{j}^{\prime}(\ell), \mathbf{i}^{\prime}\left(\ell^{m}\right)$, $\mathbf{j}^{\prime}\left(\ell^{m}\right)$ and the polarizing sequences $\mathfrak{h}_{k}^{\prime}(\ell), 1 \leq k \leq d$, and $\mathfrak{h}_{r}^{\prime}\left(\ell^{m}\right), 1 \leq r \leq d^{m}$, as computed from this new basis, coincide precisely with the original objects $\mathbf{i}(\ell), \mathbf{j}(\ell)$, $\mathbf{i}\left(\ell^{m}\right), \mathbf{j}\left(\ell^{m}\right), \mathfrak{h}_{k}(\ell), 1 \leq k \leq d, \mathfrak{h}_{r}\left(\ell^{m}\right), 1 \leq r \leq d^{m}$, respectively. To see this, note that if $n^{\prime}=n-1$ already holds, the original basis will suffice, while if $n^{\prime}=n-2$, then we take $Z_{j}^{\prime}=Z_{j}, 1 \leq j \leq n-2$, and define $Z_{n-1}^{\prime}$ and $Z_{n}^{\prime}$ according to the following. If $n-1 \notin \mathbf{e}$, then we take $Z_{n-1}^{\prime}=\mathfrak{R e}\left(Z_{n-1}\right), Z_{n}^{\prime}=\mathfrak{I m}\left(Z_{n-1}\right)$. If $n-1 \in \mathbf{e}$ and $n \notin \mathbf{e}$, then $n-1=j_{k} \in \mathbf{j}$, and we fix $\epsilon$ such that $\ell \in O_{\epsilon}$ and take

$$
Z_{n-1}^{\prime}=\phi_{n-1}^{\epsilon}(\ell)^{-1} U_{h}(\ell)=\phi_{n-1}^{\epsilon}(\ell)^{-1} \rho_{k-1}\left(\alpha_{1, k}(\ell) X_{1}+\alpha_{2, k}(\ell) X_{2}, \ell\right)
$$

and

$$
Z_{n}^{\prime}=\phi_{n-1}^{\epsilon}(\ell)^{-1} \rho_{k-1}\left(\alpha_{2, k}(\ell) X_{1}-\alpha_{1, k}(\ell) X_{2}, \ell\right) .
$$

If $n-1=j_{h} \in \mathbf{j}$ and $n=j_{k} \in \mathbf{j}$, then set

$$
Z_{n-1}^{\prime}=\phi_{n-1}^{\epsilon}(\ell)^{-1} U_{h}(\ell), Z_{n}^{\prime}=\phi_{n}^{\epsilon}(\ell)^{-1} U_{k}(\ell) .
$$

Put $\ell^{\circ}=\left.\ell\right|_{\mathfrak{s}^{\circ}}$, and let $\pi_{\circ, m}:\left(\mathfrak{s}^{\circ}\right)^{*} \rightarrow \mathfrak{s}_{m}^{*}$ be the restriction mapping. Let $\mathbf{i}^{\circ}$ be the subsequence of $\mathbf{i}$ whose values are the set $\left\{i_{k} \mid j_{k} \leq n-1\right\}$ and let $\mathbf{j}^{\circ}$ be the corresponding subsequence of $\mathbf{j}$. By the above, we have $\mathbf{i}\left(\ell^{\circ}\right)=\mathbf{i}^{\circ}$ and $\mathbf{j}\left(\ell^{\circ}\right)=\mathbf{j}^{\circ}$, and hence by induction and the observation above, we have $\mathbf{i}\left(\ell^{m}\right)=\mathbf{i}^{\prime}\left(\ell^{m}\right)=$ $\mathbf{i}\left(\pi_{\circ, m}\left(\ell^{\circ}\right)\right)=\mathbf{i}^{m}$ and $\mathbf{j}\left(\ell^{m}\right)=\mathbf{j}^{\prime}\left(\ell^{m}\right)=\mathbf{j}\left(\pi_{\circ, m}\left(\ell^{\circ}\right)\right)=\mathbf{j}^{m}$.

Now by definition of the $j_{r}, 1 \leq r \leq k_{1}-1$, and $\mathfrak{h}_{r}(\ell), 0 \leq r \leq k_{1}-1$, the relation $\mathfrak{s}_{m} \subset \mathfrak{h}_{k_{1}-1}(\ell) \subset \mathfrak{h}_{r}(\ell)$ holds so that if $r=k_{1}$,

$$
\begin{aligned}
\mathfrak{h}_{1}\left(\ell^{m}\right) & =\mathfrak{s}_{i_{k_{1}}}^{\ell} \cap \mathfrak{s}_{m}=\left(\mathfrak{s}_{i_{1}} \cap \mathfrak{h}_{k_{1}-1}(\ell)\right)^{\ell} \cap \mathfrak{h}_{k_{1}-1}(\ell) \cap \mathfrak{s}_{m} \\
& =\mathfrak{h}_{k_{1}}(\ell) \cap \mathfrak{s}_{m} .
\end{aligned}
$$

If $k_{1}<r<k_{2}$, then it is clear from the definition of $\mathfrak{h}_{r}(\ell)$ that $\mathfrak{h}_{r}(\ell) \cap \mathfrak{s}_{m}=$ $\mathfrak{h}_{k_{1}}(\ell) \cap \mathfrak{s}_{m}$. Thus, for $r=k_{2}$,

$$
\begin{aligned}
\mathfrak{h}_{2}\left(\ell^{m}\right) & =\mathfrak{h}_{1}\left(\ell^{m}\right) \cap\left(\mathfrak{h}_{1}\left(\ell^{m}\right) \cap \mathfrak{s}_{i_{k_{2}}}\right)^{\ell} \\
& =\left(\mathfrak{h}_{k_{1}}(\ell) \cap \mathfrak{s}_{m}\right) \cap\left(\mathfrak{h}_{k_{1}}(\ell) \cap \mathfrak{s}_{i_{k_{2}}}\right)^{\ell} \\
& =\left(\mathfrak{h}_{k_{2}-1}(\ell) \cap \mathfrak{s}_{m}\right) \cap\left(\mathfrak{h}_{k_{2}-1}(\ell) \cap \mathfrak{s}_{i_{k_{2}}}\right)^{\ell} \\
& =\left(\mathfrak{h}_{k_{2}-1}(\ell) \cap\left(\mathfrak{h}_{k_{2}-1}(\ell) \cap \mathfrak{s}_{i_{k_{2}}}\right)^{\ell}\right) \cap \mathfrak{s}_{m} \\
& =\mathfrak{h}_{k_{2}}(\ell) \cap \mathfrak{s}_{m} .
\end{aligned}
$$

Continuing in this way we obtain the result.

Remark 1. In a subsequent result we will show that $\varphi\left(\ell^{m}\right)=\mathbf{e}^{m} \cap \varphi(\ell)$.

Next we must clarify the relationship between objects obtained by application of Lemma 3.1 to $\ell^{m} \in \mathfrak{g}_{m}^{*}$ and $\ell \in \mathfrak{g}^{*}$. We begin with the following remark, which is easily verified with a case-by-case examination of the definitions of the objects $Z_{i_{k}}(\ell)$ above. 
Remark 2. Set $K^{m}=\left\{1,2, \ldots, d_{m}\right\}$ and define $K_{N}^{m} \subset K^{m}$ exactly as the sets $K_{N}$ for $N=0,1,2,3,4$ and 5; thus $K_{0}^{m}=\left\{1 \leq r \leq d^{m} \mid i_{r}^{m}-1 \in I\right.$ and $\left.i_{r}^{m} \in I\right\}$, etc.

The properties of the index sequences $\mathbf{i}$ and $\mathbf{j}$, and the definition (and properties) of the index sequences $\mathbf{i}^{m}$ and $\mathbf{j}^{m}$, show that $K_{N}^{m}=\left\{r \in K^{m} \mid k_{r} \in K_{N}\right\}$ if $N=0,2,3,4 a, 5$.

However, if $k \in K_{4 b}$ and $j_{k} \leq m<j_{k+1}$, then $k=k_{r} \in K\left(\mathfrak{s}_{m}\right)$ but $r \in K_{1}^{m}$. Hence the subindex sets $K_{1}^{m}$ and $K_{4 b}^{m}$ satisfy

$$
K_{1}^{m}=\left\{r \in K^{m} \mid k_{r} \in K_{1} \text { or } k_{r} \in K_{4 b} \text { and } m<j_{k_{r}+1}\right\}
$$

and

$$
K_{4 b}^{m}=\left\{r \in K^{m} \mid k_{r} \in K_{4 a} \text { and } j_{k_{r}+1} \leqslant m\right\} .
$$

Now let $k=k_{r} \in K\left(\mathfrak{s}_{m}\right)$, and consider the definitions of $Z_{i_{k}}(\ell)$ and $Z_{i_{r}^{m}}\left(\ell^{m}\right)$. If $k \in K_{0} \cup K_{3}$ or if $k \in K_{4 a} \cup K_{5 a}$, then the functions $\beta_{\epsilon, k}(\ell)$ are constant. In fact they are either 0 or 1 with their value being determined only by the particular case at hand and the value of $\epsilon$. Since these cases are the same for $i_{r}^{m}$, we have $\beta_{\epsilon, k}(\ell)=\beta_{\epsilon, r}^{m}\left(\ell^{m}\right), \epsilon=1,2$, and hence $Z_{i_{k_{r}}}(\ell)=Z_{i_{r}^{m}}(\ell)$ holds for all $\ell \in \Omega$ in each of these cases.

If $k \in K_{1} \cup K_{2}$ or if $k \in K_{4 b} \cup K_{5 b}$, then there is $k^{\prime}<k$ and, for each $\epsilon=1$, 2, a pair of elements $W_{\epsilon}, Z_{\epsilon}$, belonging to $\mathfrak{s}_{m}$ such that

$$
\beta_{\epsilon, k}(\ell)=\ell\left[W_{\epsilon}, \rho_{k^{\prime}}\left(Z_{\epsilon}, \ell\right)\right] \text {. }
$$

If $k \in K_{1}$, then $r \in K_{1}^{m}$, and we have $k^{\prime}=k-1, r^{\prime}=r-1$, and for the same elements $W_{\epsilon}, Z_{\epsilon}$,

$$
\beta_{\epsilon, r}^{m}(\ell)=\ell^{m}\left[W_{\epsilon}, \rho_{r-1}^{m}\left(Z_{\epsilon}, \ell^{m}\right)\right] .
$$

If $k \in K_{2}$, then let $h<k$ such that $j_{h} \notin I$ and $j_{h}+1=i_{k}$. From the definitions above we see that $k^{\prime}=h-1$. Now $j_{h}<m$ says that $h=k_{s} \in K^{m}$, that is, $j_{h}=j_{s}^{m}$ for some $1 \leq s<r$. It follows that $r^{\prime}=s-1$, and for the same elements $W_{\epsilon}, Z_{\epsilon}$

$$
\beta_{\epsilon, r}^{m}(\ell)=\ell^{m}\left[W_{\epsilon}, \rho_{s-1}^{m}\left(Z_{\epsilon}, \ell^{m}\right)\right] .
$$

Suppose then that $k \in K_{4 b}$. Then $r \in K_{1}^{m}$ or $r \in K_{4 b}^{m}$ exactly as $j_{k+1}>m$ or $j_{k+1} \leq m$, that is, as $k+1 \notin K^{m}$ or $k+1 \in K^{m}$. But we have already observed that the definition of the functions $\beta_{\epsilon, k}(\ell)$ is the same when $k \in K_{1}$ and $k \in K_{4 b}$. Hence in either case we have $k^{\prime}=k-1, r^{\prime}=r-1$, and

$$
\beta_{\epsilon, r}^{m}(\ell)=\ell^{m}\left[W_{\epsilon}, \rho_{r-1}^{m}\left(Z_{\epsilon}, \ell^{m}\right)\right]
$$

for the same pair of elements $W_{\epsilon}, Z_{\epsilon}$.

Finally, if $k \in K_{5 b}$, then we see that $k^{\prime}=k-2$ and $r^{\prime}=r-2$, and that

$$
\beta_{\epsilon, r}^{m}(\ell)=\ell^{m}\left[W_{\epsilon}, \rho_{r-2}^{m}\left(Z_{\epsilon}, \ell^{m}\right)\right]
$$

for the same pair of elements $W_{\epsilon}, Z_{\epsilon}$.

Lemma 5.1. For $\ell \in \Omega$ and for each $1 \leq r \leq d^{m}$, set

$$
\mathcal{V}_{r}(\ell)=\mathbb{C} \text {-span }\left\{V_{t}(\ell) \mid 1 \leq t \leq k_{r}-1, t \notin K^{m}\right\} .
$$


Then for each $\ell \in \Omega$ and $1 \leq r \leq d^{m}$, one has all of the following:

(a) $\mathcal{V}_{r}(\ell) \subset \mathfrak{s}_{m}^{\ell}$.

(b) For each $k_{r-1}<k<k_{r}$,

$$
\rho_{k}(Z, \ell)=\rho_{r-1}^{m}\left(Z, \ell^{m}\right) \bmod \mathcal{V}_{r}(\ell)
$$

holds for all $Z \in \mathfrak{s}_{m}$. Hence for $k_{r-1}<k<k_{r}$ (or if $r=1, k<k_{1}$ ),

$$
\ell\left[W, \rho_{k}(Z, \ell)\right]=\ell^{m}\left[W, \rho_{r-1}^{m}\left(Z, \ell^{m}\right)\right]
$$

holds for $W, Z \in \mathfrak{s}_{m}$.

(c) For $1 \leq r \leq d^{m}$, we have $\beta_{\epsilon, k_{r}}(\ell)=\beta_{\epsilon, r}^{m}\left(\ell^{m}\right)$ and $\alpha_{\epsilon, k_{r}}(\ell)=\alpha_{\epsilon, r}^{m}\left(\ell^{m}\right)$, and hence

$$
Z_{i_{k_{r}}}(\ell)=Z_{i_{r}^{m}}\left(\ell^{m}\right) \text { and } Z_{j_{k_{r}}}(\ell)=Z_{j_{r}^{m}}\left(\ell^{m}\right) .
$$

(d) Set $V_{k}(\ell)=\rho_{k-1}\left(Z_{i_{k}}(\ell), \ell\right)$ and $U_{k}(\ell)=\rho_{k-1}\left(Z_{j_{k}}(\ell), \ell\right), 1 \leq k \leq d$, and similarly, $V_{r}^{m}(\ell)=\rho_{r-1}^{m}\left(Z_{i_{r}^{m}}\left(\ell^{m}\right), \ell^{m}\right)$ and $U_{r}^{m}\left(\ell^{m}\right)=\rho_{r-1}^{m}\left(Z_{j_{r}^{m}}\left(\ell^{m}\right), \ell^{m}\right), 1 \leq r \leq$ $d^{m}$. Then for each $1 \leq r \leq d^{m}$ one has

$$
V_{k_{r}}(\ell)=V_{r}(\ell) \bmod \mathcal{V}_{r}(\ell) \text { and } U_{k_{r}}(\ell)=U_{r}^{m}\left(\ell^{m}\right) \bmod \mathcal{V}_{r}(\ell) .
$$

Hence for any $W \in \mathfrak{s}_{m}$ and $\ell \in \Omega$, one has $\ell\left[W, V_{k_{r}}(\ell)\right]=\ell^{m}\left[W, V_{r}^{m}\left(\ell^{m}\right)\right]$ and $\ell\left[W, U_{k_{r}}(\ell)\right]=\ell^{m}\left[W, U_{r}^{m}\left(\ell^{m}\right)\right]$.

(e) $\rho_{k_{r}}(Z, \ell)=\rho_{r}^{m}\left(Z, \ell^{m}\right) \bmod \mathcal{V}_{r}(\ell)$ holds for all $Z \in \mathfrak{s}_{m}$. Hence if $W, Z \in \mathfrak{s}_{m}$, then $\ell\left[W, \rho_{k_{r}}(Z, \ell)\right]=\ell^{m}\left[W, \rho_{r}^{m}\left(Z, \ell^{m}\right)\right]$ holds.

Proof. As before we set $Y_{1}=\mathfrak{R e} Z_{i_{k_{r}}}, Y_{2}=\mathfrak{I m} Z_{i_{k_{r}}}, X_{1}=\mathfrak{R e} Z_{j_{k_{r}}}$ and $X_{2}=$ $\mathfrak{I m} Z_{j_{k_{r}}}$.

We proceed by induction on $r$. Suppose $r=1$. To prove part $(a)$, note that $\mathcal{V}_{1}(\ell) \subset \mathfrak{s}_{i_{k_{1}}-1}$, and hence by property $(g)$ of Lemma 3.2 (applied to $\mathfrak{s}_{m}$ ) it is contained in $\mathfrak{s}_{m}^{\ell}$. Now if $k<k_{1}$, then the definition of $\rho_{k}$ shows that for any $Z \in \mathfrak{s}_{m}, \rho_{k}(Z, \ell)=\rho_{0}^{m}\left(Z, \ell^{m}\right) \bmod \mathcal{V}_{1}(\ell)$; thus $(b)$ is proved.

Now we turn to part (c) for the case $r=1$, and for this we refer to Remark 2 preceding this lemma. Let $k=k_{1}$. If $k \in K_{0} \cup K_{3}$ or if $k \in K_{4 a} \cup K_{5 a}$, then we have already observed that $Z_{i_{k_{r}}}(\ell)=Z_{i_{r}^{m}}\left(\ell^{m}\right)$. If $k \in K_{1}$, then by Remark 2, we have elements $W_{\epsilon}$ and $Z_{\epsilon}$ such that

$$
\beta_{\epsilon, k}(\ell)=\ell\left[W_{\epsilon}, \rho_{k-1}\left(Z_{\epsilon}, \ell\right)\right]
$$

and

$$
\beta_{\epsilon, r}^{m}\left(\ell^{m}\right)=\ell^{m}\left[W_{\epsilon}, \rho_{r-1}^{m}\left(Z_{\epsilon}, \ell^{m}\right)\right] .
$$

But by part (b), which we have already verified for $r=1$,

$$
\ell\left[W_{\epsilon}, \rho_{k-1}\left(Z_{\epsilon}, \ell\right)\right]=\ell^{m}\left[W_{\epsilon}, \rho_{r-1}^{m}\left(Z_{\epsilon}, \ell^{m}\right)\right] ;
$$

hence $Z_{i_{k_{r}}}(\ell)=Z_{i_{r}^{m}}\left(\ell^{m}\right)$ in this case also. If $k \in K_{4 b}$, then a repetition of the argument for $k \in K_{1}$ verbatim shows that (c) holds. Note that the properties of the index sequences $\mathbf{i}$ and $\mathbf{j}$ and the definition (and properties) of the index sequences $\mathbf{i}^{m}$ and $\mathbf{j}^{m}$ show that $k_{1} \notin K_{2} \cup K_{5}$. Hence $Z_{i_{k_{r}}}(\ell)=Z_{i_{r}^{m}}\left(\ell^{m}\right)$ is proved for $r=1$. Also for $\epsilon=1,2$, we apply the above and part (b) to get

$$
\begin{aligned}
\alpha_{\epsilon, k}(\ell) & =\beta_{1, k}(\ell) \ell\left[X_{\epsilon}, \rho_{k-1}\left(Y_{1}, \ell\right)\right]+\beta_{2, k}(\ell) \ell\left[X_{\epsilon}, \rho_{k-1}\left(Y_{2}, \ell\right)\right] \\
& =\beta_{1,1}^{m}\left(\ell^{m}\right) \ell^{m}\left[X_{\epsilon}, \rho_{0}^{m}\left(Y_{1}, \ell^{m}\right)\right]+\beta_{2,1}^{m}\left(\ell^{m}\right) \ell^{m}\left[X_{\epsilon}, \rho_{0}^{m}\left(Y_{2}, \ell^{m}\right)\right] \\
& =\alpha_{\epsilon, 1}^{m}\left(\ell^{m}\right)
\end{aligned}
$$


so $Z_{j_{k_{r}}}(\ell)=Z_{j_{r}^{m}}\left(\ell^{m}\right)$ also. Thus $(c)$ is proved for $r=1$. Now apply $(c)$ together with part (b) again:

$$
\begin{aligned}
\ell\left[W, V_{k_{1}}(\ell)\right] & =\beta_{1, k_{1}}(\ell) \ell\left[W, \rho_{k_{1}-1}\left(Y_{1}, \ell\right)\right]+\beta_{2, k_{1}}(\ell) \ell\left[W, \rho_{k_{1}-1}\left(Y_{2}, \ell\right)\right] \\
& =\beta_{1,1}^{m}(\ell) \ell\left[W, \rho_{0}^{m}\left(Y_{1}, \ell^{m}\right)\right]+\beta_{2,1}(\ell) \ell\left[W, \rho_{0}^{m}\left(Y_{2}, \ell^{m}\right)\right] \\
& =\ell^{m}\left[W, V_{1}^{m}(\ell)\right]
\end{aligned}
$$

and similarly if " $V$ " is replaced by " $U$ ". Thus part $(d)$ is proved for $r=1$.

As for (e), we compute using $(d)$ and the recursion formula for $\rho_{k_{1}}$ :

$$
\begin{aligned}
\rho_{k_{1}}(Z, \ell) & =\rho_{k_{1}-1}(Z, \ell)-\frac{\ell\left[\rho_{k_{1}-1}(Z, \ell), V_{k_{1}}(\ell)\right]}{\ell\left[U_{k_{1}}(\ell), V_{k_{1}}(\ell)\right]} U_{k_{1}}(\ell)-\frac{\ell\left[\rho_{k_{1}-1}(Z, \ell), U_{k_{1}}(\ell)\right]}{\ell\left[V_{k_{1}}(\ell), U_{k_{1}}(\ell)\right]} V_{k_{1}}(\ell) \\
& =\rho_{k_{1}-1}(Z, \ell)-\frac{\ell\left[\rho_{k_{1}-1}(Z, \ell), V_{k_{1}}(\ell)\right]}{\ell\left[U_{k_{1}}(\ell), V_{k_{1}}(\ell)\right]} U_{k_{1}}(\ell)-\frac{\ell\left[\rho_{k_{1}-1}(Z, \ell), U_{k_{1}}(\ell)\right]}{\ell\left[V_{k_{1}}(\ell), U_{k_{1}}(\ell)\right]} V_{k_{1}}(\ell) \\
& =\rho_{k_{1}-1}(Z, \ell)-\frac{\ell\left[Z, V_{k_{1}}(\ell)\right]}{\ell\left[U_{k_{1}}(\ell), V_{k_{1}}(\ell)\right]} U_{k_{1}}(\ell)-\frac{\ell\left[Z, U_{k_{1}}(\ell)\right]}{\ell\left[V_{k_{1}}(\ell), U_{k_{1}}(\ell)\right]} V_{k_{1}}(\ell) \\
& =\rho_{k_{1}-1}(Z, \ell)-\frac{\ell\left[Z, V_{1}^{m}\left(\ell^{m}\right)\right]}{\ell\left[U_{1}^{m}(\ell), V_{1}^{m}(\ell)\right]} U_{k_{1}}(\ell)-\frac{\ell\left[Z, U_{1}^{m}\left(\ell^{m}\right)\right]}{\ell\left[V_{1}^{m}(\ell), U_{1}^{m}(\ell)\right]} V_{k_{1}}(\ell),
\end{aligned}
$$

and then

$$
\begin{aligned}
\rho_{k_{1}}(Z, \ell)= & \rho_{0}^{m}\left(Z, \ell^{m}\right)-\frac{\ell^{m}\left[Z, V_{1}^{m}\left(\ell^{m}\right)\right]}{\ell^{m}\left[U_{1}^{m}\left(\ell^{m}\right), V_{1}^{m}\left(\ell^{m}\right)\right]} U_{1}^{m}\left(\ell^{m}\right) \\
& \quad-\frac{\ell^{m}\left[Z, U_{1}^{m}\left(\ell^{m}\right)\right]}{\pi(\ell)\left[V_{1}^{m}\left(\ell^{m}\right), U_{1}^{m}\left(\ell^{m}\right)\right]} V_{1}^{m}\left(\ell^{m}\right) \bmod \mathcal{V}_{1}(\ell) \\
= & \rho_{1}^{m}\left(Z, \ell^{m}\right) \bmod \mathcal{V}_{1}(\ell) .
\end{aligned}
$$

Thus (a) - (e) are verified in the case that $r=1$.

Now suppose that (a) - (e) are true for $r \geq 1$. Set $k=k_{r+1}$. If $k \in K_{5}$, then $k-1 \in K_{4}$ and $k-1 \in K^{m}$, so statement (a) is vacuous in this case. Suppose that $k \notin K_{5}$ and that $k_{r}<t<k$; then $V_{t}(\ell) \in \mathfrak{h}_{t-1}(\ell)$. More precisely, if $t \notin K_{5}$ or $t=k_{r}+1 \in K_{5}$, then $V_{t}(\ell) \in \mathfrak{h}_{t-1}(\ell)$, and if $t>k_{r}+1$ and $t \in K_{5}$, then $V_{t}(\ell) \in \mathfrak{h}_{t-2}(\ell)$. Thus by Proposition 5.1 and the definition of $i_{r+1}^{m}$,

$$
V_{t}(\ell) \in\left(\mathfrak{s}_{m} \cap \mathfrak{h}_{k_{r}}(\ell)\right) \cap \mathfrak{s}_{i_{k}^{\prime}}=\mathfrak{h}_{r}^{m}\left(\ell^{m}\right) \cap \mathfrak{s}_{i_{r+1}^{m}} \subset \mathfrak{h}_{r}^{m}\left(\ell^{m}\right)^{\ell}
$$

Now

$$
\mathfrak{s}_{m}=\mathfrak{h}_{r}^{m}\left(\ell^{m}\right)+\operatorname{span}\left\{U_{1}^{m}\left(\ell^{m}\right), \ldots, U_{r-1}^{m}\left(\ell^{m}\right)\right\},
$$

and by induction (parts (a), (c) and $(e))$ we have $\mathcal{V}_{r}(\ell) \subset \mathfrak{s}_{m}^{\ell}$ and

$$
U_{s}^{m}\left(\ell^{m}\right)=U_{k_{s}}(\ell) \bmod \mathcal{V}_{r}(\ell), 1 \leq s \leq r-1 .
$$

It follows that $V_{t}(\ell) \in\left\{U_{1}^{m}\left(\ell^{m}\right), \ldots, U_{r-1}^{m}\left(\ell^{m}\right)\right\}^{\ell}$, and hence

$$
V_{t}(\ell) \in\left(\mathfrak{h}_{r}^{m}\left(\ell^{m}\right)\right)^{\ell} \cap\left\{U_{1}^{m}\left(\ell^{m}\right), \ldots, U_{r-1}^{m}\left(\ell^{m}\right)\right\}^{\ell} \subset \mathfrak{s}_{m}^{\ell} .
$$


Thus

$$
\mathcal{V}_{r+1}(\ell) \subset \mathfrak{s}_{m}^{\ell}
$$

and (a) is proved for $r+1$. Now if $Z \in \mathfrak{s}_{m}$, then by induction (part $(e)$ ) we have

$$
\rho_{r}^{m}\left(Z, \ell^{m}\right)=\rho_{k_{r}}(Z, \ell) \bmod \mathcal{V}_{r}(\ell)
$$

Now $\rho_{k_{r}}(Z, \ell) \in \mathfrak{s}_{m}$, and by (a) $V_{t}(\ell) \in \mathfrak{s}_{m}{ }^{\ell}$ holds for all $k_{r}<t<k_{r+1}$. Hence the recursion formula for $\rho_{k}$ shows that

$$
\rho_{k}(Z, \ell)=\rho_{r}^{m}\left(Z, \ell^{m}\right) \bmod \mathcal{V}_{r+1}(\ell) .
$$

Thus (b) is proved for $r+1$.

Turning to part (c), we first prove the equation $\beta_{\epsilon, k_{r+1}}(\ell)=\beta_{\epsilon, r+1}^{m}(\ell)$. To this end, we begin by noting that if $k_{r+1} \in K_{0} \cup K_{3} \cup K_{4 a} \cup K_{5 a}$, then the equation is clear (see the remark above). Setting $k=k_{r+1}$ and $Y_{1}=\mathfrak{R e} Z_{i_{k}}, Y_{2}=\mathfrak{I m} Z_{i_{k}}$, we address the remaining cases as follows.

Suppose $k \in K_{1} \cup K_{4 b}$. Recall that in this case, $r+1 \in K_{1}^{m} \cup K_{4 b}^{m}$ also. Here we apply part (b) (or by induction, part (e) if $k-1=k_{r}$ ) to get

$$
\beta_{\epsilon, k}(\ell)=\ell\left[Z_{j_{k}}, \rho_{k-1}\left(Y_{\epsilon}, \ell\right)\right]=\ell^{m}\left[Z_{j_{k}}, \rho_{r}^{m}\left(Y_{\epsilon}, \ell^{m}\right)\right]=\beta_{\epsilon, r+1}^{m}\left(\ell^{m}\right) .
$$

Suppose $k \in K_{2}$. Recall this means that $i_{k}-1 \notin I$ and $i_{k}-1=j_{h}$ for some $h<k$. Now necessarily $h \in K^{m}$, so for some $s<r+1, h=k_{s}$. Now in this case

$$
\beta_{\epsilon, k}(\ell)=\beta_{1, h}(\ell) \ell\left[X_{\epsilon}^{\prime}, \rho_{h-1}\left(Y_{1}^{\prime}, \ell\right)\right]+\beta_{2, h}(\ell) \ell\left[X_{\epsilon}^{\prime}, \rho_{h-1}\left(Y_{2}^{\prime}, \ell\right)\right]
$$

where we have set $X_{1}^{\prime}=\mathfrak{R e} Z_{j_{h}}, X_{2}^{\prime}=\mathfrak{I m} Z_{j_{h}}, Y_{1}^{\prime}=\mathfrak{R e} Z_{i_{h}}$, and $Y_{2}^{\prime}=\mathfrak{I m} Z_{j_{h}}$. By induction (part $(c)$ ) we have $\beta_{\epsilon, h}(\ell)=\beta_{\epsilon, s}^{m}\left(\ell^{m}\right), \epsilon=1,2$, and by induction (part (b) or part $(e))$ we have $\ell\left[X_{\epsilon}^{\prime}, \rho_{h-1}\left(Y_{\delta}^{\prime}, \ell\right)\right]=\ell^{m}\left[X_{\epsilon}^{\prime}, \rho_{s-1}^{m}\left(Y_{\delta}^{\prime}, \ell^{m}\right)\right], \epsilon, \delta=1,2$. Thus $\beta_{\epsilon, k}(\ell)=\beta_{\epsilon, r+1}^{m}\left(\ell^{m}\right)$.

Suppose $k \in K_{5 b}$. Here $r+1 \in K_{5 b}^{m}$ also, and $\beta_{\epsilon, k}(\ell)= \pm \beta_{\epsilon+1, k-1}(\ell)=$ $\pm \beta_{\epsilon+1, r}^{m}\left(\ell^{m}\right)=\beta_{\epsilon, r+1}^{m}\left(\ell^{m}\right)$ (wherein addition mod 2 is used in $\epsilon+1$ ).

This concludes the proof that $\beta_{\epsilon, k}(\ell)=\beta_{\epsilon, r+1}^{m}\left(\ell^{m}\right)$. As for $\alpha_{\epsilon, k}$, recall that in every case

$$
\alpha_{\epsilon, k}(\ell)=\beta_{1, k}(\ell) \ell\left[X_{\epsilon}, \rho_{k-1}\left(Y_{1}, \ell\right)\right]+\beta_{2, k}(\ell) \ell\left[X_{\epsilon}, \rho_{k-1}\left(Y_{2}, \ell\right)\right]
$$

with a similar formula for $\alpha_{\epsilon, r+1}^{m}\left(\ell^{m}\right)$. Using the equality $\beta_{\epsilon, k}(\ell)=\beta_{\epsilon, r+1}^{m}\left(\ell^{m}\right)$ already established, together with induction, part $(b)$, we find that $\alpha_{\epsilon, k}(\ell)=$ $\alpha_{\epsilon, r+1}^{m}(\ell)$. This concludes the proof of part (c) for $r+1$.

Now (d) follows easily from the preceding: with $k=k_{r+1}$,

$$
\begin{aligned}
V_{k}(\ell) & =\rho_{k-1}\left(Z_{i_{k}}(\ell), \ell\right)=\rho_{k-1}\left(Z_{i_{r+1}^{m}}^{m}\left(\ell^{m}\right), \ell\right) \\
& =\rho_{r}^{m}\left(Z_{i_{r+1}^{m}}^{m}\left(\ell^{m}\right), \ell^{m}\right) \bmod \mathcal{V}_{r+1}(\ell) \\
& =V_{r+1}^{m}\left(\ell^{m}\right) \bmod \mathcal{V}_{r+1}(\ell)
\end{aligned}
$$


Finally, the proof of part (e) for $r+1$ is parallel to the calculation for $r=1$. Recall that $k=k_{r+1}$ :

$$
\begin{aligned}
\rho_{k}(Z, \ell) & =\rho_{k-1}(Z, \ell)-\frac{\ell\left[\rho_{k-1}(Z, \ell), V_{k}(\ell)\right]}{\ell\left[X_{k}(\ell), V_{k}(\ell)\right]} U_{k}(\ell)-\frac{\ell\left[\rho_{k-1}(Z, \ell), U_{k}(\ell)\right]}{\ell\left[V_{k}(\ell), U_{k}(\ell)\right]} V_{k}(\ell) \\
& =\rho_{k-1}(Z, \ell)-\frac{\ell\left[Z, V_{k}(\ell)\right]}{\ell\left[U_{k}(\ell), V_{k}(\ell)\right]} U_{k}(\ell)-\frac{\ell\left[Z, U_{k}(\ell)\right]}{\ell\left[V_{k}(\ell), U_{k}(\ell)\right]} V_{k}(\ell) \\
& =\rho_{k-1}(Z, \ell)-\frac{\ell\left[Z, V_{r+1}^{m}\left(\ell^{m}\right)\right]}{\ell\left[U_{r+1}^{m}(\ell), V_{r+1}^{m}(\ell)\right]} U_{k}(\ell)-\frac{\ell\left[Z, U_{r+1}^{m}\left(\ell^{m}\right)\right]}{\ell\left[V_{r+1}^{m}(\ell), U_{r+1}^{m}(\ell)\right]} V_{k}(\ell) \\
& =\rho_{r}^{m}\left(Z, \ell^{m}\right)-\frac{\ell^{m}\left[Z, V_{r+1}^{m}\left(\ell^{m}\right)\right]}{\ell^{m}\left[U_{r+1}^{m}\left(\ell^{m}\right), V_{r+1}^{m}\left(\ell^{m}\right)\right]} U_{r+1}^{m}\left(\ell^{m}\right) \\
& \quad-\frac{\ell^{m}\left[Z, U_{r+1}^{m}\left(\ell^{m}\right)\right]}{\ell^{m}\left[V_{r+1}^{m}\left(\ell^{m}\right), U_{r+1}^{m}\left(\ell^{m}\right)\right]} V_{r+1}^{m}\left(\ell^{m}\right) \bmod \mathcal{V}_{r+1}(\ell) \\
& =\rho_{r+1}^{m}\left(Z, \ell^{m}\right) \bmod \mathcal{V}_{r+1}(\ell) .
\end{aligned}
$$

This completes the induction step and the proof.

The following is almost immediate.

Theorem 5.2 (Projection of layers and cross-sections). Let $\mathfrak{g}$ be an exponential Lie algebra and choose an adaptable basis for $\mathfrak{s}=\mathfrak{g}_{\mathbb{C}}$. Let $\Omega=\Omega_{\mathbf{e}, \mathbf{j}, \varphi} \subset \mathfrak{g}^{*}$ be an ultrafine layer, and fix $m \in I$. Define $\varphi^{m}=\varphi \cap \mathbf{e}^{m}$. Then $\varphi\left(\ell^{m}\right)=\varphi^{m}$ holds for each $\ell$ in $\Omega$, and hence $\pi_{m}(\Omega) \subset \Omega^{m}$, where $\Omega^{m}=\Omega_{\mathbf{e}^{m}, \mathbf{j}^{m}, \varphi^{m}}$ is the corresponding ultrafine layer in $\mathfrak{g}_{m}^{*}$. Let $\Sigma$ and $\Sigma^{m}$ be the adaptable cross-sections for coadjoint orbits in $\Omega$ and $\Omega^{m}$, respectively. Then $\pi_{m}(\Sigma) \subset \Sigma^{m}$.

Proof. Let $\ell \in \Omega$ and let $j=i_{k_{r}}$ be a value of the index sequence $\mathbf{i}^{m}$. Then by Lemma 5.1 $U_{r}^{m}\left(\ell^{m}\right)=U_{k_{r}}(\ell) \bmod \mathcal{V}_{r}(\ell)$, and since $\mathcal{V}_{r}(\ell) \subset C_{j}$, we have

$$
\gamma_{j}\left(U_{r}^{m}\left(\ell^{m}\right)\right)=\gamma_{j}\left(U_{k_{r}}(\ell)\right) .
$$

Proposition 4.1 now shows that $\varphi\left(\ell^{m}\right)=\varphi^{m}$. From Proposition 5.1 we have $\pi_{m}(\Omega) \subset \Omega^{m}$.

Now given $\ell \in \Sigma$, the preceding shows that $\ell^{m} \in \Omega^{m}$, and Lemma 5.1] shows that for $j \in \mathbf{e}^{m} \backslash \varphi^{m}$, we have $j \in \mathbf{e} \backslash \varphi$ and

$$
\ell^{m}\left(Z_{j}^{m}\left(\ell^{m}\right)\right)=\ell^{m}\left(Z_{j}(\ell)\right)=\ell\left(Z_{j}(\ell)\right)=0 .
$$

On the other hand, if $j=i_{k_{r}} \in \varphi^{m}$, then $j \in \varphi$, and again Lemma 5.1 gives

$$
\mathbf{b}_{j}^{m}\left(\ell^{m}\right)=\frac{\gamma_{j}\left(U_{r}^{m}\left(\ell^{m}\right)\right)}{\ell^{m}\left[Z_{j}, U_{r}^{m}\left(\ell^{m}\right)\right]}=\frac{\gamma_{j}\left(U_{k}(\ell)\right)}{\ell\left[Z_{j}, U_{k}(\ell)\right]}=\mathbf{b}_{j}(\ell)
$$

so that $\left|\mathbf{b}_{j}^{m}\left(\ell^{m}\right)\right|=\left|\mathbf{b}_{j}(\ell)\right|=1$. Hence by Theorem 4.2 applied to $\Sigma^{m}$, we have $\ell^{m} \in \Sigma^{m}$.

Corollary 5.3. Let $m \in I$, let $\Omega$ be an ultrafine layer, let $\Omega^{m}$ be the ultrafine layer in $\mathfrak{g}_{m}^{*}$ that contains $\pi_{m}(\Omega)$ and write $K\left(\mathfrak{s}_{m}\right)=\left\{k_{1}<k_{2}<\cdots<k_{d^{m}}\right\}$. Let $O_{\epsilon}$ be a covering set for $\Omega$ and define $\epsilon^{m} \in\{1,2\}^{d^{m}}$ by $\epsilon_{r}^{m}=\epsilon_{k_{r}}$. Then $\pi_{m}\left(O_{\epsilon}\right) \subset O_{\epsilon^{m}}$. Moreover, $\phi_{i_{k_{r}}}^{\epsilon}(\ell)=\phi_{i_{r}^{m}}^{\epsilon^{m}}\left(\ell^{m}\right)$ holds for all $\ell \in O_{\epsilon}$.

Proof. By Theorem 5.2 we have $\pi^{m}(\Omega) \subset \Omega^{m}$, and by Lemma 5.1 part (c), we have $\beta_{\epsilon_{k_{r}}, k_{r}}(\ell)=\beta_{\epsilon_{r}^{m}, r}(\ell)$. Hence the result follows from the definitions of $O_{\epsilon}$ and $O_{\epsilon^{m}}$. 


\section{Construction of $q$-Functions}

As always $\mathfrak{g}$ is a real exponential solvable Lie algebra, and an adaptable basis $\left(Z_{1}, Z_{2}, \ldots, Z_{n}\right)$ for $\mathfrak{s}=\mathfrak{g}_{c}$ is in place. In this section we begin to develop the induction step by which the canonical coordinates will be constructed. We retain all notation, definitions and constructions of the preceding. Set $m=n^{\prime}$ and recall that our definition of adaptable basis permits the possibility that $m=n-2$ (whence it would follow that $\mathfrak{s}$ is nilpotent). Let $\Omega$ be an ultrafine layer in $\mathfrak{g}^{*}$ with crosssection $\Sigma$, and let $\Omega^{m}$ be the ultrafine layer in $\mathfrak{g}_{m}^{*}$ with cross-section $\Sigma^{m}$ such that $\pi_{m}(\Omega) \subset \Omega^{m}$ and $\pi_{m}(\Sigma) \subset \Sigma^{m}$. Assume that orbits in $\Omega$ have dimension $2 d>0$. Set $\mathbf{n}=\{m+1, n\}$. We assume for the remainder of this section that $\mathbf{n} \cap \mathbf{e} \neq \emptyset$, so that $\operatorname{dim}\left(\mathcal{O}_{\ell}\right)>\operatorname{dim}\left(\mathcal{O}_{\ell^{m}}\right)$ then holds for all $\ell \in \Omega$. Naturally $\mathbf{n} \cap \mathbf{e}$ consists of either one or two indices, and it may or may not be the case that a value of the sequence $\mathbf{i}=\left(i_{k}\right)$ is included. Because $i_{k}<j_{k}$ holds for each, however, there are only three main cases:

Case (1): $\mathbf{n} \cap \mathbf{e}=\left\{j_{k}\right\}$ for some $1 \leq k \leq d$.

Case (2): $\mathbf{n} \cap \mathbf{e}=\left\{j_{h}, j_{k}\right\}$ for some $1 \leq h<k \leq d$.

Case (3): $\mathbf{n} \cap \mathbf{e}=\left\{i_{d}, j_{d}\right\}$.

With an analysis of these cases and subcases within them, one observes that the structural relationship between an orbit $\mathcal{O}$ in $\Omega$ and $G_{m}$-orbits in $\pi_{m}(\mathcal{O})$ remains constant throughout $\Omega$.

Lemma 6.1. One has $\mathfrak{s}(\ell)=\mathfrak{s}_{m}\left(\ell^{m}\right)$ for all $\ell \in \Omega$ if and only if Case (3) holds.

Proof. Suppose that $n-1=i_{d}$. Since both $n-1$ and $n$ are jump indices, we have $\mathfrak{s}(\ell) \subset \mathfrak{s}_{m}$, hence $\mathfrak{s}(\ell) \subset \mathfrak{s}_{m}\left(\ell^{m}\right)$. On the other hand, if $Z \in \mathfrak{s}_{m}\left(\ell^{m}\right)$, then by the definition of $\rho_{d-1}(\cdot, \ell)$ and part $(g)$ of Lemma $3.2, Z=\rho_{d-1}(Z, \ell) \in \rho_{d-1}\left(\mathfrak{s}_{m}, \ell\right) \subset$ $\mathfrak{s}(\ell)$.

Now suppose that $i_{d}<n-1$. If every jump index is smaller than $n-1$, then for each $\ell \in \Omega$ we have that $\rho_{d}\left(Z_{n-1}, \ell\right)$ and $\rho_{d}\left(Z_{n}, \ell\right)$ belong to $\mathfrak{s}(\ell)$ and not $\mathfrak{s}_{m}\left(\ell^{m}\right)$. On the other hand, if $n-1$ is a jump index, then $n-1=j_{k}$ for some $1 \leq k \leq d$, and then by Lemma 5.1, we have that $V_{k}(\ell)$ belongs to $\mathfrak{s}_{m}\left(\ell^{m}\right)$ but not $\mathfrak{s}(\ell)$.

The method by which we treat Case (3) is quite distinct from the first two cases, and we defer this treatment until the next section.

The induction step by canonical coordinates as constructed in [2] hinges upon the definition of the "new $q$ function" by means of equation (1.2). We propose to adapt that step to the present setting. In what follows we shall show existence of, and obtain an explicit expression for, these function(s) (two are needed in Case (2)). In this section we address Cases (1) and (2), and we shall see that, despite a large number of cases for arrangement of the jump indices, only one elementary expression is needed. In Case (3), we shall also obtain a precise expression for $q$, but by a different method.

We adopt the following simplifications of notation: we denote $\pi_{m}$ by $\pi$, and for any function $F$ defined on (a subset of) $\mathfrak{s}_{m}^{*}$, we write $F(\ell)$ in place of $\pi^{*} F(\ell)=$ $F(\pi(\ell))$.

Proposition 6.1. For an ultrafine layer with orbits of dimension $2 d>0$ and with $\mathbf{n} \cap \mathbf{e} \neq \emptyset$, we have the following. 
(1) If $\mathbf{n} \cap \mathbf{e}=\left\{j_{k}\right\}$, then for each $\ell \in \Omega$ there exists a unique complex number $q(\ell)$ such that $q(\ell) Z_{j_{k}}(\ell)$ belongs to $\mathfrak{g}$ and

$$
\sigma^{m}\left(\exp \left(q(\ell) Z_{j_{k}}(\ell)\right) \ell\right)=\pi(\sigma(\ell)) .
$$

(2) If $\mathbf{n} \cap \mathbf{e}=\left\{j_{h}, j_{k}\right\}$ with $h<k$, then for each $\ell \in \Omega$ there exists a unique pair $\left(q_{1}(\ell), q_{2}(\ell)\right)$ of complex numbers such that $q_{1}(\ell) Z_{j_{h}}(\ell)$ and $q_{2}(\ell) Z_{j_{k}}(\ell)$ belong to $\mathfrak{g}$ and

$$
\sigma^{m}\left(\exp \left(q_{1}(\ell) Z_{j_{h}}(\ell)\right) \exp \left(q_{2}(\ell) Z_{j_{k}}(\ell)\right) \ell\right)=\pi(\sigma(\ell)) .
$$

Proof. Suppose first that we are in Case (1), and set $j=j_{k}$. If $m=n-1$, then the argument is exactly the same as [1, Lemma 2.1]. If $m=n-2, \mathfrak{g}$ is nilpotent. We choose $\epsilon \in\{1,2\}^{d}$ such that $\mathcal{O} \subset O_{\epsilon}$. By Lemma 3.3. $O_{\epsilon}, Z_{j}(\ell), \phi_{j}^{\epsilon}(\ell)$ are $G$-invariant. Set $X^{\epsilon}(\ell)=\phi_{j}^{\epsilon}(\ell)^{-1} Z_{j}(\ell), \mathfrak{g}^{\mathcal{O}}=\mathfrak{g}_{m}+\mathbb{R} X^{\epsilon}(\ell)$. We can then apply the argument of [1, Lemma 2.1] to obtain for each $\ell \in \Omega$ a unique real number $u^{\epsilon}(\ell)$ such that

$$
\exp \left(u^{\epsilon}(\ell) X^{\epsilon}(\ell)\right) \ell \in \mathcal{O}_{\pi(\sigma(\ell))} .
$$

We set $q(\ell)=\phi_{j}^{\epsilon}(\ell) u^{\epsilon}(\ell)$.

Suppose now that we are in Case (2). In this case we have $m=n-2$, so that $\mathfrak{g}$ is nilpotent, and as in the preceding case for each $\ell \in \Omega$ we define $X_{1}^{\epsilon}(\ell)=$ $\phi_{j_{h}}^{\epsilon}(\ell)^{-1} Z_{j_{h}}(\ell), \mathfrak{g}^{\mathcal{O}}=\mathfrak{g}_{m}+\mathbb{R} X_{1}^{\epsilon}(\ell)$, and $X_{2}^{\epsilon}(\ell)=\phi_{j_{k}}^{\epsilon}(\ell)^{-1} Z_{j_{k}}(\ell)$. Let $\pi^{\mathcal{O}}$ be the restriction map onto $\left(\mathfrak{g}^{\mathcal{O}}\right)^{*}$. Again following the argument of [1, Lemma 2.1], let $u_{2}^{\epsilon}(\ell)$ be the unique real number for which

$$
\ell^{\prime}=\exp \left(u_{2}^{\epsilon}(\ell) X_{2}^{\epsilon}(\ell)\right) \ell \in \mathcal{O}_{\pi \mathcal{O}}(\sigma(\ell)),
$$

and let $u_{1}^{\epsilon}(\ell)$ the unique real number for which

$$
\exp \left(u_{1}^{\epsilon}(\ell) X_{1}^{\epsilon}(\ell)\right) \ell^{\prime} \in \mathcal{O}_{\pi_{m}(\sigma(\ell))} .
$$

We set $q_{1}(\ell)=\phi_{j_{h}}^{\epsilon}(\ell) u_{1}^{\epsilon}(\ell)$ and $q_{2}(\ell)=\phi_{j_{k}}^{\epsilon}(\ell) u_{2}^{\epsilon}(\ell)$.

We now proceed to show that these functions are real analytic and to derive an expression for them. We begin with some preliminary and somewhat technical results.

Recall that for an open subset $O$ of $\Omega, \mathcal{A}(O)$ denotes the set of all complex-valued functions $f$ on $O$ that are real analytic on $O$ in the sense of Definition 6. For each $1 \leq j \leq n$, set

$$
\mathcal{A}_{j}(O)=\left\{f \in \mathcal{A}(O) \mid f \text { depends only upon } \ell_{1}, \ell_{2}, \ldots, \ell_{j}\right\} .
$$

The following lemma follows easily from results of [4].

Lemma 6.2. Let $j=i_{k}$ be a value of the index sequence $\mathbf{i}$, and let $V \in \mathfrak{s}$. Then for each $0 \leq r \leq k-1$, the function $\gamma_{j}\left(\rho_{r}(V, \ell)\right)$ belongs to $\mathcal{A}_{j-1}(\Omega)$ and the function $\ell \mapsto \ell\left[Z_{j}, \rho_{r}(V, \ell)\right]$ has the form

$$
\ell\left[Z_{j}, \rho_{r}(V, \ell)\right]=\gamma_{j}\left(\rho_{r}(V, \ell)\right) \ell_{j} \bmod \mathcal{A}_{j-1}(\Omega) .
$$

Proof. It is clear that $\ell \mapsto \ell\left[Z_{j}, \rho_{r}(V, \ell)\right]$ and $\ell \mapsto \gamma_{j}\left(\rho_{r}(V, \ell)\right)$ belong to $\mathcal{A}(\Omega)$. From [4, Lemma 1.3.7], we have that $\gamma_{j}\left(\rho_{r}(V, \ell)\right)$ depends only upon $\ell_{1}, \ldots, \ell_{j-1}$. That $\ell\left[Z_{j}, \rho_{r}(V, \ell)\right]$ has the expression above follows from a repetition (indeed a simplification) of the proof of [4, Lemma 1.3.10], where instead of $j<i_{k}$ we assume that $j=i_{k}$. 
Corollary 6.2. Suppose that $j=e_{a}$ belongs to $\mathbf{e} \backslash \varphi$. If $j=j_{k}$ (resp. $j=i_{k}$ ), then $\ell \mapsto \ell\left[Z_{j}, V_{k}(\ell)\right]$ (resp. $\ell \mapsto \ell\left[Z_{j}, U_{k}(\ell)\right]$ ) belongs to $\mathcal{A}_{j-1}(\Omega)$. Moreover, for each $\epsilon \in\{1,2\}^{d}$, the functions $\zeta_{a}^{\epsilon}(\ell)$ and $\phi_{j}^{\epsilon}(\ell)$ belong to $\mathcal{A}_{j-1}\left(O_{\epsilon}\right)$.

Proof. Suppose that $j=j_{k}$; from [4, Lemma 1.3.4] we have $\ell \mapsto \ell\left[Z_{j}, V_{k}(\ell)\right] \in$ $\mathcal{A}_{i_{k}}(\Omega)$. The rest of the result now follows from an examination of the definitions of the functions $\zeta_{a}^{\epsilon}(\ell)$ and $\phi_{j}^{\epsilon}(\ell)$.

Suppose that $j=i_{k}$. Observe that since $j \notin \varphi$, then $\gamma_{j}\left(\rho_{k-1}\left(Z_{j_{k}}, \ell\right)\right)=0$. Hence by Lemma 6.2 we have that $\ell\left[Z_{j}, \rho_{k-1}\left(\mathfrak{R e} Z_{j_{k}}, \ell\right)\right]$ and $\ell\left[Z_{j}, \rho_{k-1}\left(\mathfrak{I m} Z_{j_{k}}, \ell\right)\right]$ depend only upon $\ell_{1}, \ldots, \ell_{j-1}$, from which it follows that $\ell\left[\bar{Z}_{j}, \rho_{k-1}\left(\Re \mathfrak{R e} Z_{j_{k}}, \ell\right)\right]$ and $\ell\left[\bar{Z}_{j}, \rho_{k-1}\left(\mathfrak{I m} Z_{j_{k}}, \ell\right)\right]$ depend only upon $\ell_{1}, \ldots, \ell_{j-1}$, whence the same can be said of $\beta_{1, k}(\ell), \beta_{2, k}(\ell), \alpha_{1, k}(\ell)$, and $\alpha_{2, k}(\ell)$. It follows that $\ell\left[Z_{j}, U_{k}(\ell)\right]$ depends only upon $\ell_{1}, \ldots, \ell_{j-1}$. The result again follows from examination of the definitions of the functions $\zeta_{a}^{\epsilon}(\ell)$ and $\phi_{j}^{\epsilon}(\ell)$.

Lemma 6.3. Let $m$ be an index belonging to $I$ and let $j=i_{k}=e_{a}$ be an index smaller than $m$ such that $i_{r}=i_{r}^{m}, 1 \leq r \leq k-1$, and $j_{r}=j_{r}^{m}, 1 \leq r \leq k-1$. Then $\left(g^{m}\right)^{a-1}(\ell)=g^{a-1}(\ell)$ holds for each $\ell \in \Omega$.

Proof. Referring to the notation of Lemma 5.1, we have $\mathcal{V}_{r}(\ell)=(0), 1 \leq r \leq k-1$, and so from parts $(c)$ and $(d)$ of Lemma 5.1 we have $U_{r}(\ell)=U_{r}^{m}\left(\ell^{m}\right), V_{r}(\ell)=$ $V_{r}^{m}\left(\ell^{m}\right), 1 \leq r \leq k-1$, and for any covering set $O_{\epsilon}$ in $\Omega$,

$$
r_{b}^{\epsilon}(\ell)=r_{b}^{\epsilon^{m}}\left(\ell^{m}\right), 1 \leq b \leq a-1 .
$$

Hence the orbits $G \cdot \pi_{j^{\prime}}(\ell)$ and $G_{m} \cdot \pi_{j^{\prime}}(\ell)$ coincide. This holds for each $\ell \in \Omega$, so we see that the actions of $G$ and $G^{m}$ are the same on $\pi_{j^{\prime}}(\Omega)$ so that the quotient spaces $\pi_{j^{\prime}}(\Omega) / G$ and $\pi_{j^{\prime}}(\Omega) / G_{m}$ are identical. Now by Theorem $5.2 \pi_{m}(\Omega) \subset \Omega^{m}$, hence $\pi_{j^{\prime}}(\Omega) \subset \pi_{j^{\prime}}\left(\Omega^{m}\right)$, while, $\pi_{m}(\Sigma) \subset \Sigma^{m}$, and hence $\pi_{j^{\prime}}(\Sigma) \subset \pi_{j^{\prime}}\left(\Sigma^{m}\right)$. By Theorem 5.2 we have that $\pi_{j^{\prime}}(\Sigma)$ is a cross-section for $G$-orbits in $\pi_{j^{\prime}}(\Omega)$, while $\pi_{j^{\prime}}\left(\Sigma^{m}\right)$ is a cross-section for $G_{m}$-orbits in $\pi_{j^{\prime}}(\Omega)$. It follows that $\pi_{j^{\prime}}(\Sigma)=\pi_{j^{\prime}}\left(\Sigma^{m}\right)$.

Now by Theorem 5.2

$$
\begin{aligned}
g^{a-1}(\ell) \pi_{j^{\prime}}(\ell) & =\pi_{j^{\prime}}(\Sigma) \cap G \cdot \pi_{j^{\prime}}(\ell) \\
& =\pi_{j^{\prime}}\left(\Sigma^{m}\right) \cap G^{m} \cdot \pi_{j^{\prime}}(\ell)=\left(g^{m}\right)^{a-1}\left(\ell^{m}\right) \pi_{j^{\prime}}(\ell) .
\end{aligned}
$$

Using Lemma 4.4 and $(\star)$ we get $g^{a-1}(\ell)=\left(g^{m}\right)^{a-1}\left(\ell^{m}\right)$.

Lemma 6.4. Suppose that $k \in K_{1}$, set $j=i_{k}=e_{a}$ and assume that $j \notin \varphi$. Suppose that $q$ is a complex-valued function on $\Omega$ which satisfies $q(\ell) U_{k}(\ell) \in \mathfrak{g}$ and for which there is $Y(\ell) \in \mathcal{A}_{j-1}(\Omega)$ such that

$$
\ell_{j}-q(\ell) \ell\left[U_{k}(\ell), Z_{j}\right]=\zeta_{a}^{\epsilon}(\ell) i \mathfrak{I m}\left(\zeta_{a}^{\epsilon}(\ell)^{-1} \ell_{j}\right)+Y(\ell)
$$

for every covering set $O_{\epsilon}$. Then $q \in \mathcal{A}_{j}(\Omega)$ and

$$
q(\ell)=\frac{\ell\left(Z_{i_{k}}(\ell)\right)}{\ell\left[U_{k}(\ell), Z_{i_{k}}(\ell)\right]} \bmod \mathcal{A}_{j-1}(\Omega) .
$$

Proof. From Corollary 6.2 we have $\zeta_{a}^{\epsilon}(\ell) \in \mathcal{A}_{j-1}\left(O_{\epsilon}\right)$. The given equation is equivalent to

$$
\zeta_{a}^{\epsilon}(\ell) \mathfrak{R e}\left(\zeta_{a}^{\epsilon}(\ell)^{-1} \ell_{j}\right)-q(\ell) \ell\left[U_{k}(\ell), Z_{j}\right]=Y(\ell)
$$


and since $\ell \mapsto 1 / \ell\left[U_{k}(\ell), Z_{j}\right]$ belongs to $\mathcal{A}_{j-1}(\Omega)$ (see the proof of Corollary 6.2), we get

$$
q(\ell)=\frac{\zeta_{a}^{\epsilon}(\ell) \mathfrak{R e}\left(\zeta_{a}^{\epsilon}(\ell)^{-1} \ell_{j}\right)}{\ell\left[U_{k}(\ell), Z_{j}\right]} \bmod \mathcal{A}_{j-1}(\Omega) .
$$

Now since

$$
\zeta_{a}^{\epsilon}(\ell)=\frac{\ell\left[Z_{j}, \phi_{j}^{\epsilon}(\ell)^{-1} U_{k}(\ell)\right]}{\left|\ell\left[Z_{j}, \phi_{j}^{\epsilon}(\ell)^{-1} U_{k}(\ell)\right]\right|}
$$

our expression for $q(\ell)$ becomes

$$
\begin{aligned}
q(\ell) & =\phi_{j}^{\epsilon}(\ell)^{-1} \mathfrak{R e}\left(\frac{\ell_{j}}{\ell\left[\phi_{j}^{\epsilon}(\ell)^{-1} U_{k}(\ell), Z_{j}\right]}\right) \\
& =\phi_{j}^{\epsilon}(\ell)^{-1} \frac{1}{2}\left(\frac{\ell_{j}}{\ell\left[\phi_{j}^{\epsilon}(\ell)^{-1} U_{k}(\ell), Z_{j}\right]}+\frac{\bar{\ell}_{j}}{\ell\left[\phi_{j}^{\epsilon}(\ell)^{-1} U_{k}(\ell), \bar{Z}_{j}\right]}\right) \\
& =\frac{1}{2}\left(\frac{\ell_{j}}{\ell\left[U_{k}(\ell), Z_{j}\right]}+\frac{\bar{\ell}_{j}}{\ell\left[U_{k}(\ell), \bar{Z}_{j}\right]}\right) \bmod \mathcal{A}_{j-1}(\Omega) .
\end{aligned}
$$

Now recalling the definition of $Z_{j}(\ell)$, we have

$$
Z_{j}(\ell)=\frac{1}{2}\left(b_{1}(\ell) Z_{j}+b_{2}(\ell) \bar{Z}_{j}\right)
$$

where $b_{1}(\ell)=\ell\left[\rho_{k-1}\left(Z_{j_{k}}, \ell\right), \bar{Z}_{j}\right]$ and $b_{2}(\ell)=\ell\left[\rho_{k-1}\left(Z_{j_{k}}, \ell\right), Z_{j}\right]$. Similarly

$$
Z_{j_{k}}(\ell)=\frac{1}{2}\left(a_{1}(\ell) Z_{j_{k}}+a_{2}(\ell) \bar{Z}_{j_{k}}\right),
$$

where $a_{1}(\ell)=\ell\left[\rho_{k-1}\left(\bar{Z}_{j_{k}}, \ell\right), Z_{j}(\ell)\right]$ and $a_{2}(\ell)=\ell\left[\rho_{k-1}\left(Z_{j_{k}}, \ell\right), Z_{j}(\ell)\right]$. Set

$$
\check{Z}_{j}(\ell)=\frac{1}{2 i}\left(b_{1}(\ell) Z_{j}-b_{2}(\ell) \bar{Z}_{j}\right) .
$$

A straightforward calculation shows that

$$
\ell\left[U_{k}(\ell), \check{Z}_{j}(\ell)\right]=\frac{1}{4 i}\left(\left|b_{1}(\ell)\right|^{2}-\left|b_{2}(\ell)\right|^{2}\right) .
$$

But since we are in the case where $k \in K_{1}$, we have

$$
\mathfrak{I m}\left(\beta_{k, 1}(\ell) \overline{\beta_{k, 2}(\ell)}\right)=0 .
$$

Since $b_{1}(\ell)=\beta_{k, 1}(\ell)-i \beta_{k, 2}(\ell)$ and $b_{2}(\ell)=\beta_{k, 1}(\ell)+i \beta_{k, 2}(\ell)$, we have $\left|b_{1}\right|^{2}=\left|b_{2}\right|^{2}$ and hence $\ell\left[U_{k}(\ell), \check{Z}_{j}(\ell)\right]=0$. Now since $Z_{j}(\ell)+i \check{Z}_{j}(\ell)=b_{1}(\ell) Z_{j}$ and $Z_{j}(\ell)-$ $i \check{Z}_{j}(\ell)=b_{2}(\ell) \bar{Z}_{j}$, we have

$$
\ell\left[U_{k}(\ell), Z_{j}(\ell)\right]=b_{1}(\ell) \ell\left[U_{k}(\ell), Z_{j}\right]=b_{2}(\ell) \ell\left[U_{k}(\ell), \bar{Z}_{j}\right] .
$$

Substituting into our expression for $q(\ell)$, we obtain

$$
\begin{aligned}
q(\ell) & =\frac{1}{2}\left(\frac{\ell_{j}}{\ell\left[U_{k}(\ell), Z_{j}\right]}+\frac{\bar{\ell}_{j}}{\ell\left[U_{k}(\ell), \bar{Z}_{j}\right]}\right) \\
& =\frac{1}{2}\left(\frac{b_{1}(\ell) \ell_{j}}{\ell\left[U_{k}(\ell), Z_{j}(\ell)\right]}+\frac{b_{2}(\ell) \bar{\ell}_{j}}{\ell\left[U_{k}(\ell), Z_{j}(\ell)\right]}\right) \\
& =\frac{\ell\left(Z_{j}(\ell)\right)}{\ell\left[U_{k}(\ell), Z_{j}(\ell)\right]} \bmod \mathcal{A}_{j-1}(\Omega) .
\end{aligned}
$$


Lemma 6.5. Suppose that $k \in K_{2}$, set $j=j_{h}=i_{k}-1=e_{a}$ and suppose that $q(\ell)$ is a complex-valued function on $\Omega$ for which $q(\ell) U_{k}(\ell) \in \mathfrak{g}$ and for which there is $Y(\ell)$ in $\mathcal{A}_{j-1}(\Omega)$ such that

$$
\zeta_{a}^{\epsilon}(\ell) \mathfrak{I m}\left(\zeta_{a}^{\epsilon}(\ell)^{-1}\left(\ell_{j}-q(\ell) \ell\left[U_{k}(\ell), Z_{j}\right]\right)\right)=Y(\ell),
$$

for any covering set $O_{\epsilon}$. Then (just as in Lemma 6.4) we have

$$
q(\ell)=\frac{\ell\left(Z_{i_{k}}(\ell)\right)}{\ell\left[U_{k}(\ell), Z_{i_{k}}(\ell)\right]} \bmod \mathcal{A}_{j-1}(\Omega) .
$$

Proof. Again by Corollary 6.2, the functions $\phi_{j}^{\epsilon}(\ell)$ and $\zeta_{a}^{\epsilon}(\ell)$ belong to $\mathcal{A}_{j-1}\left(O_{\epsilon}\right)$. In contrast with the case where $k \in K_{1}$, the formula for $Z_{i_{k}}(\ell)$ here is

$$
Z_{i_{k}}(\ell)=\check{Z}_{j}(\ell)=\frac{1}{2 i}\left(a_{1}(\ell)-a_{2}(\ell)\right)
$$

where $a_{1}(\ell)=\ell\left[\bar{Z}_{j}, V_{h}(\ell)\right]$ and $a_{2}(\ell)=\ell\left[Z_{j}, V_{h}(\ell)\right]$. Now in this case

$$
\zeta_{a}^{\epsilon}(\ell)=\frac{\ell\left[Z_{j}, \phi_{j}^{\epsilon}(\ell)^{-1} V_{h}(\ell)\right]}{\left|\ell\left[Z_{j}, \phi_{j}^{\epsilon}(\ell)^{-1} V_{h}(\ell)\right]\right|}=\frac{\phi_{j}^{\epsilon}(\ell)^{-1} a_{2}(\ell)}{\left|a_{2}(\ell)\right|}
$$

so that the given equation becomes

$$
\begin{aligned}
Y(\ell) & \left.=\zeta_{a}^{\epsilon}(\ell) \mathfrak{I m}\left(\zeta_{a}^{\epsilon}(\ell)^{-1} \ell_{j}\right)-\zeta_{a}^{\epsilon}(\ell) \mathfrak{I m}\left(\zeta_{a}^{\epsilon}(\ell)^{-1} q(\ell) \ell\left[U_{k}(\ell), Z_{j}\right]\right)\right) \\
& =\phi_{j}^{\epsilon}(\ell)^{-1} a_{2}(\ell) \mathfrak{I m}\left(\frac{\ell_{j}}{\phi_{j}^{\epsilon}(\ell)^{-1} a_{2}(\ell)}\right)-\phi_{j}^{\epsilon}(\ell)^{-1} a_{2}(\ell) \mathfrak{I m}\left(q(\ell) \frac{\ell\left[U_{k}(\ell), Z_{j}\right]}{\phi_{j}^{\epsilon}(\ell)^{-1} a_{2}(\ell)}\right) .
\end{aligned}
$$

Since $\phi_{j}^{\epsilon}(\ell)^{-1} V_{h}(\ell)$ is real, then $\overline{\phi_{j}^{\epsilon}(\ell)^{-1} a_{2}(\ell)}=\phi_{j}^{\epsilon}(\ell)^{-1} a_{1}(\ell)$, so the first term in the preceding is

$$
\begin{aligned}
\phi_{j}^{\epsilon}(\ell)^{-1} a_{2}(\ell) & \mathfrak{I m}\left(\frac{\ell_{j}}{\phi_{j}^{\epsilon}(\ell)^{-1} a_{2}(\ell)}\right) \\
& =\phi_{j}^{\epsilon}(\ell)^{-1} a_{2}(\ell) \frac{1}{2 i}\left(\frac{\ell_{j}}{\phi_{j}^{\epsilon}(\ell)^{-1} a_{2}(\ell)}-\frac{\overline{\ell_{j}}}{\phi_{j}^{\epsilon}(\ell)^{-1} a_{1}(\ell)}\right)
\end{aligned}
$$

or

$$
\begin{aligned}
\phi_{j}^{\epsilon}(\ell)^{-1} a_{2}(\ell) \mathfrak{I m}\left(\frac{\ell_{j}}{\phi_{j}^{\epsilon}(\ell)^{-1} a_{2}(\ell)}\right) & =a_{2}(\ell) \frac{1}{2 i}\left(\frac{\ell_{j}}{a_{2}(\ell)}-\frac{\overline{\ell_{j}}}{a_{1}(\ell)}\right) \\
& =\frac{1}{a_{1}(\ell)} \frac{1}{2 i}\left(a_{1}(\ell) \ell_{j}-a_{2}(\ell) \overline{\ell_{j}}\right) \\
& =\frac{\ell\left(Z_{i_{k}}(\ell)\right)}{a_{1}(\ell)} .
\end{aligned}
$$


On the other hand, using the fact that $q(\ell) U_{k}(\ell)$ is also real, together with our expression for $Z_{i_{k}}(\ell)$ above, the second term is

$$
\begin{aligned}
\phi_{j}^{\epsilon}(\ell)^{-1} a_{2}(\ell) & \mathfrak{I m}\left(\frac{\ell\left[q(\ell) U_{k}(\ell), Z_{j}\right]}{\phi_{j}^{\epsilon}(\ell)^{-1} a_{2}(\ell)}\right) \\
& =\phi_{j}^{\epsilon}(\ell)^{-1} a_{2}(\ell) \frac{1}{2 i}\left(\frac{\ell\left[q(\ell) U_{k}(\ell), Z_{j}\right]}{\phi_{j}^{\epsilon}(\ell)^{-1} a_{2}(\ell)}-\frac{\ell\left[q(\ell) U_{k}(\ell), \bar{Z}_{j}\right]}{\phi_{j}^{\epsilon}(\ell)^{-1} a_{1}(\ell)}\right) \\
& =q(\ell) a_{2}(\ell) \frac{1}{2 i}\left(\frac{\ell\left[U_{k}(\ell), Z_{j}\right]}{a_{2}(\ell)}-\frac{\ell\left[U_{k}(\ell), \bar{Z}_{j}\right]}{a_{1}(\ell)}\right) \\
& =q(\ell) \frac{1}{a_{1}(\ell)} \frac{1}{2 i}\left(a_{1}(\ell) \ell\left[U_{k}(\ell), Z_{j}\right]-a_{2}(\ell) \ell\left[U_{k}(\ell), \bar{Z}_{j}\right]\right) \\
& =q(\ell) \frac{\ell\left[U_{k}(\ell), Z_{i_{k}}(\ell)\right]}{a_{1}(\ell)} .
\end{aligned}
$$

Combining these new expressions for $Y(\ell)$ in the given equation we now have

$Y(\ell)=\frac{\ell\left(Z_{i_{k}}(\ell)\right)}{a_{1}(\ell)}-q(\ell) \frac{\ell\left[U_{k}(\ell), Z_{i_{k}}(\ell)\right]}{a_{1}(\ell)}=\frac{1}{a_{1}(\ell)}\left(\ell\left(Z_{i_{k}}(\ell)\right)-q(\ell) \ell\left[U_{k}(\ell), Z_{i_{k}}(\ell)\right]\right)$.

Since $a_{1}(\ell) / \ell\left[U_{k}(\ell), Z_{i_{k}}(\ell)\right]$ belongs to the algebra $\mathcal{A}_{j-1}(\Omega)$, the desired equation is obtained.

Lemma 6.6. Suppose that $k \in K_{5 b}$, set $j=i_{k}-1=i_{k-1}=e_{a}$ and suppose that $q(\ell)$ is a complex-valued function on $\Omega$ for which $q(\ell) U_{k}(\ell) \in \mathfrak{g}$ and for which there is $Y(\ell) \in \mathcal{A}_{j-1}(\Omega)$ such that

$$
\zeta_{a}^{\epsilon}(\ell) \mathfrak{I m}\left(\zeta_{a}^{\epsilon}(\ell)^{-1}\left(\ell_{j}-q(\ell) \ell\left[U_{k}(\ell), Z_{j}\right]\right)\right)=Y(\ell)
$$

for any covering set $O_{\epsilon}$. Then (just as in Lemma 6.4) one has

$$
q(\ell)=\frac{\ell\left(Z_{i_{k}}(\ell)\right)}{\ell\left[U_{k}(\ell), Z_{i_{k}}(\ell)\right]} \bmod \mathcal{A}_{j-1}(\Omega) .
$$

Proof. In this case we have $Z_{j}(\ell)=\frac{1}{2}\left(b_{1}(\ell) Z_{j}+b_{2}(\ell) \bar{Z}_{j}\right)$ and

$$
Z_{i_{k}}(\ell)=\check{Z}_{j}(\ell)=\frac{1}{2 i}\left(b_{1}(\ell) Z_{j}-b_{2}(\ell) \bar{Z}_{j}\right),
$$

where $b_{1}(\ell)=\ell\left[\rho_{k-1}\left(Z_{j_{k}}, \ell\right), \bar{Z}_{j}\right]$ and $b_{2}(\ell)=\ell\left[\rho_{k-1}\left(Z_{j_{k}}, \ell\right), Z_{j}\right]$.

Set $a_{1}(\ell)=\ell\left[\bar{Z}_{j_{k}}, V_{k-1}(\ell)\right]$ and $a_{2}(\ell)=\ell\left[Z_{j_{k}}, V_{k-1}(\ell)\right], c_{1}(\ell)=\ell\left[\bar{Z}_{j}, U_{k-1}(\ell)\right]$ and $c_{2}(\ell)=\ell\left[Z_{j}, U_{k-1}(\ell)\right]$. As in the proof of Lemma 6.4 we have

$$
Z_{j}(\ell)+i \check{Z}_{j}(\ell)=b_{1}(\ell) Z_{j}
$$

and

$$
Z_{j}(\ell)-i \check{Z}_{j}(\ell)=b_{2}(\ell) \bar{Z}_{j} .
$$

Also, this time by construction, we have

$$
\begin{aligned}
\ell\left[U_{k-1}(\ell), \check{Z}_{j}(\ell)\right] & =\ell\left[U_{k-1}(\ell), Z_{i_{k}}(\ell)\right]=\ell\left[U_{k-1}(\ell), \rho_{k-2}\left(Z_{i_{k}}(\ell), \ell\right)\right] \\
& =\ell\left[U_{k-1}(\ell), V_{k}(\ell)\right]=0 .
\end{aligned}
$$

Hence

$$
\ell\left[U_{k-1}(\ell), Z_{j}(\ell)\right]=-b_{1}(\ell) c_{2}(\ell)=-b_{2}(\ell) c_{1}(\ell) .
$$


With this background, we consider the given equation. We have

$$
\zeta_{a}^{\epsilon}(\ell)=\frac{\ell\left[Z_{j}, \phi_{j}^{\epsilon}(\ell)^{-1} U_{k-1}(\ell)\right]}{\left|\ell\left[Z_{j}, \phi_{j}^{\epsilon}(\ell)^{-1} U_{k-1}(\ell)\right]\right|}=\frac{\phi_{j}^{\epsilon}(\ell)^{-1} c_{2}(\ell)}{\left|c_{2}(\ell)\right|}
$$

so the given equation becomes

$$
Y(\ell)=\phi_{j}^{\epsilon}(\ell)^{-1} c_{2}(\ell)\left\{\mathfrak{I m}\left(\frac{\ell_{j}}{\phi_{j}^{\epsilon}(\ell)^{-1} c_{2}(\ell)}\right)-\mathfrak{I m}\left(\frac{q(\ell) \ell\left[U_{k}(\ell), Z_{j}\right]}{\phi_{j}^{\epsilon}(\ell)^{-1} c_{2}(\ell)}\right)\right\} .
$$

Since $\phi_{j}^{\epsilon}(\ell)^{-1} U_{k-1}(\ell)$ is real, then $\overline{\phi_{j}^{\epsilon}(\ell)^{-1} c_{2}(\ell)}=\phi_{j}^{\epsilon}(\ell)^{-1} c_{1}(\ell)$. Using this and the equation for $\ell\left[U_{k-1}(\ell), Z_{j}(\ell)\right]$ above, the first term in the preceding is

$$
\begin{aligned}
\phi_{j}^{\epsilon}(\ell)^{-1} c_{2}(\ell) & \mathfrak{I m}\left(\frac{\ell_{j}}{\phi_{j}^{\epsilon}(\ell)^{-1} c_{2}(\ell)}\right) \\
& =\phi_{j}^{\epsilon}(\ell)^{-1} c_{2}(\ell) \frac{1}{2 i}\left(\frac{\ell_{j}}{\phi_{j}^{\epsilon}(\ell)^{-1} c_{2}(\ell)}-\frac{\overline{\ell_{j}}}{\phi_{j}^{\epsilon}(\ell)^{-1} c_{1}(\ell)}\right) \\
& =c_{2}(\ell) \frac{1}{2 i}\left(\frac{\ell_{j}}{c_{2}(\ell)}-\frac{\overline{\ell_{j}}}{c_{1}(\ell)}\right) \\
& =-c_{2}(\ell) \frac{1}{\ell\left[U_{k-1}(\ell), Z_{j}(\ell)\right]} \frac{1}{2 i}\left(b_{1}(\ell) \ell_{j}-b_{2}(\ell) \overline{\ell_{j}}\right) \\
& =\frac{-c_{2}(\ell)}{\ell\left[U_{k-1}(\ell), Z_{j}(\ell)\right]}\left(Z_{i_{k}}(\ell)\right) .
\end{aligned}
$$

Turning to the second term, we have

$$
\begin{aligned}
\phi_{j}^{\epsilon}(\ell)^{-1} c_{2}(\ell) & \mathfrak{I m}\left(\frac{q(\ell) \ell\left[U_{k}(\ell), Z_{j}\right]}{\phi_{j}^{\epsilon}(\ell)^{-1} c_{2}(\ell)}\right) \\
& =\phi_{j}^{\epsilon}(\ell)^{-1} c_{2}(\ell)\left(\frac{\ell\left[q(\ell) U_{k}(\ell), Z_{j}\right]}{\phi_{j}^{\epsilon}(\ell)^{-1} c_{2}(\ell)}-\frac{\ell\left[q(\ell) U_{k}(\ell), \bar{Z}_{j}\right]}{\phi_{j}^{\epsilon}(\ell)^{-1} c_{1}(\ell)}\right) \\
& =\frac{-c_{2}(\ell)}{\ell\left[U_{k-1}(\ell), Z_{j}(\ell)\right]} \frac{1}{2 i}\left(b_{1}(\ell) \ell\left[q(\ell) U_{k}(\ell), Z_{j}\right]-b_{2}(\ell) \ell\left[q(\ell) U_{k}(\ell), \bar{Z}_{j}\right]\right) \\
& =\frac{-c_{2}(\ell)}{\ell\left[U_{k-1}(\ell), Z_{j}(\ell)\right]} q(\ell) \ell\left[U_{k}(\ell), Z_{i_{k}}(\ell)\right] .
\end{aligned}
$$

Combining these simplifications we obtain

$$
Y(\ell)=\frac{-c_{2}(\ell)}{\ell\left[U_{k-1}(\ell), Z_{j}(\ell)\right]}\left(\ell\left(Z_{i_{k}}(\ell)\right)-q(\ell) \ell\left[U_{k}(\ell), Z_{i_{k}}(\ell)\right]\right) .
$$

Since $\ell\left[U_{k-1}(\ell), Z_{j}(\ell)\right] / \ell\left[U_{k}(\ell), Z_{i_{k}}(\ell)\right] c_{2}(\ell)$ belongs to $\mathcal{A}_{j-1}(\Omega)$, the desired formula follows.

We are now ready to prove the main results of this section.

Proposition 6.3. Suppose that $\mathbf{n} \cap \mathbf{e}=\left\{j_{k}\right\}$. Let $q: \Omega \rightarrow \mathbb{C}$ be the unique function for which $q(\ell) Z_{j_{k}}(\ell) \in \mathfrak{g}$ and

$$
\sigma^{m}\left(\exp \left(q(\ell) Z_{j_{k}}(\ell)\right) \ell\right)=\pi(\sigma(\ell))
$$


hold for each $\ell$. If $i_{k} \notin \varphi$, then

$$
q(\ell)=\frac{\ell\left(Z_{i_{k}}(\ell)\right)}{\ell\left[U_{k}(\ell), Z_{i_{k}}(\ell)\right]} \bmod \mathcal{A}_{i_{k}^{\prime}}(\Omega)
$$

while if $i_{k} \in \varphi$, then

$$
q(\ell)=\frac{\ln \left|\mathbf{b}_{i_{k}}(\ell)\right|}{\mathfrak{R e}\left(\gamma_{i_{k}}\left(U_{k}(\ell)\right)\right.} \bmod \mathcal{A}_{i_{k}^{\prime}}(\Omega) .
$$

Proof. As was the case in [1, Lemma 4.4], the method of proof is based upon looking at the $i_{k}$-component of the cross-section maps for both $\Omega$ and $\Omega^{m}$. For ease of notation set $j=i_{k}^{\prime}+1$.

First suppose that $i_{k} \notin \varphi$, and recall that from Propsition 4.1 this means that $\gamma_{j}\left(U_{k}(\ell)\right)=0$, which is equivalent to $\gamma_{j}\left(\rho_{k-1}\left(Z_{j_{k}}, \ell\right)\right)=0$. We consider two possibilities for the index $i_{k}$ : (a) $i_{k}-1 \in I$, or (b) $i_{k}-1 \notin I$.

Case (a). In this case $k \in K_{0} \cup K_{1}$. Set $j=e_{a}$, and set $\mu(\ell)=\mu_{j}\left(g^{a-1}(\ell)\right)$, $\ell \in \Omega$. It follows from [4, Lemma 1.3.7] and Corollary 4.8 that $\mu(\ell)$ belongs to $\mathcal{A}_{j-1}(\Omega)$. By [4, Corollary 1.3.14] we have a function $Y(\ell)$ depending only upon $\ell_{1}, \ldots, \ell_{j-1}$ such that, for each covering set $O_{\epsilon}$,

$$
\sigma_{j}(\ell)=\mu(\ell) \zeta_{a}^{\epsilon}(\ell) i \mathfrak{I m}\left(\zeta_{a}^{\epsilon}(\ell)^{-1} \ell_{j}\right)+Y(\ell)
$$

holds for all $\ell \in O_{\epsilon}$. It follows from Theorem 4.7 that $Y \in \mathcal{A}_{j-1}(\Omega)$. If $k \in K_{0}$, then $\ell_{j}$ and $\zeta_{a}^{\epsilon}(\ell)$ are real, and we take $Y=0$, so that $\sigma_{j}(\ell)=0$.

Now the definition of $q$ implies that

$$
\sigma_{j}^{m}\left(\exp \left(q(\ell) Z_{j_{k}}(\ell)\right) \ell\right)=\sigma_{j}(\ell)
$$

where $\sigma^{m}: \Omega^{m} \rightarrow \Sigma^{m}$ is the cross-section mapping for $G_{m}$-orbits in $\Omega^{m}$. Also, since $U_{r}(\ell)$ and $V_{r}(\ell)$ belong to $\mathfrak{s}_{m}$ for all $1 \leq r \leq k-1$, we have

$$
Z_{j_{k}}(\ell)=\rho_{k-1}\left(Z_{j_{k}}(\ell), \ell\right)=U_{k}(\ell) \bmod \mathfrak{s}_{m}
$$

Hence we have

$$
\sigma_{j}^{m}\left(\exp \left(q(\ell) U_{k}(\ell)\right) \ell\right)=\sigma_{j}(\ell)
$$

Applying [4, Corollary 1.3.14], Theorem 4.7 and Corollary 4.8 to $G_{m}$, we see that $\sigma^{m}$ has the form

$$
\sigma_{j}^{m}(\ell)=\mu^{m}(\ell) \ell_{j}+Y^{m}(\ell)
$$

where $\mu^{m}(\ell)$ and $Y^{m}$ belong to $\mathcal{A}_{j-1}(\Omega)$. Now

$$
\mu^{m}(\ell)=\mu_{j}\left(\left(g^{a-1}\right)^{m}(\ell)\right),
$$

and by Lemma 6.3 we have $\left(g^{a-1}\right)^{m}(\ell)=g^{a-1}(\ell)$; hence $\mu^{m}(\ell)=\mu(\ell)$ holds for each $\ell \in \Omega$. Substituting into the equality $\sigma_{j}^{m}\left(\exp \left(q(\ell) Z_{j_{k}}(\ell)\right) \ell\right)=\sigma_{j}(\ell)$, we have

$$
\mu(\ell)\left(\ell_{j}-q(\ell) \ell\left[U_{k}(\ell), Z_{j}\right]\right)+Y^{m}(\ell)=\mu(\ell) \zeta_{a}^{\epsilon}(\ell) i \mathfrak{I m}\left(\zeta_{a}^{\epsilon}(\ell)^{-1} \ell_{j}\right)+Y(\ell)
$$

and hence

$$
\ell_{j}-q(\ell) \ell\left[U_{k}(\ell), Z_{j}\right]=\zeta_{a}^{\epsilon}(\ell) i \mathfrak{I m}\left(\zeta_{a}^{\epsilon}(\ell)^{-1} \ell_{j}\right)+\frac{Y(\ell)-Y^{m}(\ell)}{\mu(\ell)} .
$$

Now by Lemma 6.4 the desired expression for $q(\ell)$ is obtained.

Case (b). Here we set $j=i_{k}-1=e_{a}$ (recall that the definition of e requires that $i_{k}-1 \in \mathbf{e}$ ). We must consider two subcases: (b1) $j=j_{h}$ with $h<k$, and (b2) $j=i_{h}$ with $h=k-1$. Before branching the argument, we observe that in either 
subcase, $j \in \mathbf{e}^{m}$. Hence by [4, Corollary 1.3.14], we have $\sigma_{j}(\ell)=0$ (because $j$ and $j^{\prime \prime}=i_{k}$ both belong to $\mathbf{e}$ ), while $\sigma_{j}^{m}(\ell)$ has the form

$$
\sigma_{j}^{m}(\ell)=\zeta_{a}^{m, \epsilon^{m}}(\ell) \mu^{m}(\ell) i \mathfrak{I m}\left(\zeta_{a}^{m, \epsilon^{m}}(\ell)^{-1} \ell_{j}\right)+Y^{m}\left(\ell_{1}, \ldots, \ell_{j-1}\right) .
$$

Here $O_{\epsilon^{m}}^{m}$ is a covering set for $\Omega^{m}$ that contains $\pi_{m}(\ell)$.

Subcase (b1). Here we have $k \in K_{2}$ and aim to show that Lemma 6.5] applies to $q(\ell)$ here. In this case we have

$$
\zeta_{a}^{m, \epsilon^{m}}(\ell)=\frac{\phi_{h}^{m, \epsilon^{m}}(\ell)^{-1} \ell\left[Z_{j}, V_{h}^{m}(\ell)\right]}{\left|\ell\left[Z_{j}, V_{h}^{m}(\ell)\right]\right|} .
$$

Now the subindex set $K^{m}$ is $K \backslash\{k\}$, and with the notation of Remark 2 following Proposition 5.1, $h=k_{h}$, and so by Lemma 5.1, part (d), we have $\ell\left[Z_{j}, V_{h}^{m}(\ell)\right]=$ $\ell\left[Z_{j}, V_{h}(\ell)\right]$. By Corollary [5.3, we have that $\phi_{h}^{m, \epsilon^{m}}(\ell)=\phi_{h}^{\epsilon}(\ell)$ holds for $\ell \in O_{\epsilon}$, where $O_{\epsilon}$ is any covering set in $F$ for which $O_{\epsilon} \subset \pi^{-1}\left(O_{\epsilon^{m}}^{m}\right)$. Hence we have $\zeta_{a}^{m, \epsilon^{m}}(\ell)=\zeta_{a}^{\epsilon}(\ell)$, and we may write

$$
\sigma_{j}^{m}(\ell)=\mu^{m}(\ell) \zeta_{a}^{\epsilon}(\ell) i \mathfrak{I m}\left(\zeta_{a}^{\epsilon}(\ell)^{-1} \ell_{j}\right)+Y^{m}\left(\ell_{1}, \ldots, \ell_{j-1}\right) .
$$

Because we have $\sigma_{j}(\ell)=0$, the definition of $q$ says that $q(\ell)$ satisfies

$$
\sigma_{j}^{m}\left(\exp \left(q(\ell) U_{k}(\ell)\right) \ell\right)=0 .
$$

Now since $j \notin \varphi$, we have $\gamma_{j}\left(U_{k}(\ell)\right)=0$. Hence $\sigma_{j}^{m}\left(\exp \left(q(\ell) U_{k}(\ell)\right) \ell\right)=0$ implies

$$
\zeta_{a}^{\epsilon}(\ell) \mathfrak{I m}\left(\zeta^{\epsilon}(\ell)^{-1}\left(\ell_{j}-q(\ell) \ell\left[U_{k}(\ell), Z_{j}\right]\right)\right)=-\frac{1}{i} \mu^{m}(\ell)^{-1} Y^{m}(\ell) .
$$

Since $\mu^{m}(\ell)^{-1}$ belongs to $\mathcal{A}_{j-1}(\Omega)$, we are in a position to apply Lemma 6.5, and the result follows.

Subcase (b2). Here we have $k \in K_{5}$, and recalling that we are in the situation $\mathbf{n} \cap \mathbf{e}=\left\{j_{k}\right\}$, we have $h=k-1 \in K_{4 b}$ with $j_{h} \leq m$ and $h=k_{r} \in K^{m}$. In this case, using Lemma 5.1.

$$
\zeta_{a}^{m, \epsilon^{m}}(\ell)=\frac{\ell\left[Z_{j}, \phi_{h}^{m, \epsilon^{m}}(\ell)^{-1} U_{h}(\ell)\right]}{\left|\ell\left[Z_{j}, U_{h}(\ell)\right]\right|} .
$$

Arguing as in Subcase (b1) we see that $\zeta_{a}^{m, \epsilon^{m}}(\ell)=\zeta_{a}^{\epsilon}(\ell)$, where $O_{\epsilon}$ is a covering set with $O_{\epsilon} \subset \pi^{-1}\left(O_{\epsilon^{m}}^{m}\right)$, and that as before we may write

$$
\sigma_{j}^{m}(\ell)=\mu^{m}(\ell) \zeta_{a}^{\epsilon}(\ell) i \mathfrak{I m}\left(\zeta_{a}^{\epsilon}(\ell)^{-1} \ell_{j}\right)+Y^{m}\left(\ell_{1}, \ldots, \ell_{j-1}\right) .
$$

Once again, we must solve $\sigma_{j}^{m}\left(\exp \left(q(\ell) U_{k}(\ell)\right) \ell\right)=0$, and, as before, this implies that

$$
\zeta^{\epsilon}(\ell) \mathfrak{I m}\left(\zeta^{\epsilon}(\ell)^{-1}\left(\ell_{j}-q(\ell) \ell\left[U_{k}(\ell), Z_{j}\right]\right)\right)=-\frac{1}{i} \mu^{m}(\ell)^{-1} Y^{m}\left(\ell_{1}, \ldots, \ell_{j-1}\right),
$$

so that now Lemma 6.6 applies.

Now suppose that $i_{k} \in \varphi$, so that $\gamma_{i_{k}}\left(U_{k}(\ell)\right) \neq 0$. Then $j_{k}>p$, for otherwise $U_{k} \in \mathfrak{s}_{p}$ : the nilradical of $\mathfrak{s}$ (part (i) of Lemma 3.1) whence $\gamma_{i_{k}}\left(U_{k}(\ell)\right)=0$. The definition of adaptable basis says that $Z_{j_{k}}=\bar{Z}_{j_{k}}$, and for ease of notation we put $X=Z_{j_{k}}$ in what follows.

From Lemma 4.2 we have that $k \in K_{0} \cup K_{1}$. Looking at the definitions of $\beta_{1, k}(\ell)$ and $\beta_{2, k}(\ell)$ in the cases $k \in K_{0}$ and $k \in K_{1}$, we see that $\beta_{1, k}(\ell)$ and $\beta_{2, k}(\ell)$ are real, and hence the function $\alpha_{1, k}(\ell)$ is real and

$$
Z_{j_{k}}(\ell)=\alpha_{1, k}(\ell) X
$$


belongs to $\mathfrak{g}$. We conclude from this that $q(\ell)$ is a real function, and that $U_{k}(\ell)=$ $\alpha_{1, k}(\ell) \rho_{k-1}(X, \ell)$ belongs to $\mathfrak{g}$ for all $\ell \in \Omega$.

By Corollary 4.8 we have an analytic function $g^{m}: \Omega^{m} \rightarrow G_{m}$ such that $\sigma^{m}(\ell)=$ $g^{m}(\ell) \ell$. Now

$$
\pi_{j^{\prime \prime}}\left(\sigma^{m}(\ell)\right)=\pi_{j^{\prime \prime}}\left(g^{m}(\ell) \ell\right)=g^{m}(\ell) \pi_{j^{\prime \prime}}(\ell)=\left(g^{m}\right)^{a-1}(\ell) \pi_{j^{\prime \prime}}(\ell),
$$

and $\left(g^{m}\right)^{a-1}(\ell)$ belongs to $\mathcal{A}_{j-1}(\Omega)$. Set $\ell^{\prime}=\exp \left(q(\ell) U_{k}(\ell)\right) \ell$. Then we have $\pi_{j-1}\left(\ell^{\prime}\right)=\pi_{j-1}(\ell)$, and hence $\left(g^{m}\right)^{a-1}\left(\ell^{\prime}\right)=\left(g^{m}\right)^{a-1}(\ell)$. It follows that

$$
\pi_{j^{\prime \prime}}\left(\sigma^{m}\left(\exp \left(q(\ell) U_{k}(\ell)\right) \ell\right)\right)=\pi_{j^{\prime \prime}}\left(\sigma^{m}\left(\ell^{\prime}\right)\right)=\left(g^{m}\right)^{a-1}\left(\ell^{\prime}\right) \ell^{\prime}=\left(g^{m}\right)^{a-1}(\ell) \ell^{\prime} .
$$

Since this element belongs to $\pi_{j^{\prime \prime}}(\Sigma)$, we have

$$
\left|\mathbf{b}_{j}\left(\left(g^{m}\right)^{a-1}(\ell) \ell^{\prime}\right)\right|=\left|\mathbf{b}_{j}\left(\left(g^{m}\right)^{a-1}(\ell) \exp \left(q(\ell) U_{k}(\ell)\right) \ell\right)\right|=1 .
$$

But the function $\mathbf{b}_{j}$ is a semi-invariant function with weight $\mu_{j}^{-1}$, so our relation yields

$$
1=\left|\mathbf{b}_{j}\left(\left(g^{m}\right)^{a-1}(\ell) \exp \left(q(\ell) U_{k}(\ell)\right) \ell\right)\right|=\mu_{j}\left(\left(g^{m}\right)^{a-1}(\ell)\right) e^{-q(\ell) \Re \mathfrak{R}\left(\gamma_{j}\left(U_{k}(\ell)\right)\right)}\left|\mathbf{b}_{j}(\ell)\right| .
$$

Since $\mu_{j}\left(\left(g^{m}\right)^{a-1}(\ell)\right)$ belongs to $\mathcal{A}_{j-1}(\Omega)$, we have

$$
q(\ell) \mathfrak{R e}\left(\gamma_{j}\left(U_{k}(\ell)\right)=\ln \left(\mu_{j}\left(\left(g^{m}\right)^{a-1}(\ell)\right)\left|\mathbf{b}_{j}(\ell)\right|\right)=\ln \left|\mathbf{b}_{j}(\ell)\right| \bmod \mathcal{A}_{j-1}(\Omega) .\right.
$$

By Lemma 6.2. the function $\ell \mapsto \mathfrak{R e}\left(\gamma_{i_{k}}\left(U_{k}(\ell)\right)\right)^{-1}$ belongs to $\mathcal{A}_{j-1}(\Omega)$ also, and the desired expression for $q(\ell)$ follows.

With the next result we address the situation where $\mathbf{n}=\left\{j_{h}, j_{k}\right\}$ with $1 \leq h<$ $k \leq d$. Note that in this case $\mathfrak{s}$ is nilpotent, so that the formulae for $\sigma$ and $\sigma^{m}$ used in the proof will be slightly simpler and the index set $\varphi$ is empty.

Proposition 6.4. Suppose that $\mathbf{n}=\left\{j_{h}, j_{k}\right\}$, with $h<k$. Then

and

$$
q_{1}(\ell)=\frac{\ell\left(Z_{i_{h}}(\ell)\right)}{\ell\left[U_{h}(\ell), Z_{i_{h}}(\ell)\right]} \bmod \mathcal{A}_{i_{h}^{\prime}}(\Omega)
$$

$$
q_{2}(\ell)=\frac{\ell\left(Z_{i_{k}}(\ell)\right)}{\ell\left[U_{k}(\ell), Z_{i_{k}}(\ell)\right]} \bmod \mathcal{A}_{i_{k}^{\prime}}(\Omega) .
$$

Proof. For ease of notation set $Z_{1}(\ell)=Z_{j_{h}}(\ell)$ and $Z_{2}(\ell)=Z_{j_{k}}(\ell)$. By Corollary 4.3 we have both $U_{h}(\ell)=Z_{1}(\ell) \bmod \mathfrak{s}_{m}$ and $U_{k}(\ell)=Z_{2}(\ell) \bmod \mathfrak{s}_{m}$. Observe that since $[\mathfrak{s}, \mathfrak{s}] \subset \mathfrak{s}_{m}$,

$$
\sigma^{m}\left(\exp \left(q_{1}(\ell) Z_{1}(\ell)\right) \exp \left(q_{2}(\ell) Z_{2}(\ell)\right) \ell\right)=\sigma^{m}\left(\exp \left(q_{2}(\ell) Z_{2}(\ell)\right) \exp \left(q_{1}(\ell) Z_{1}(\ell)\right) \ell\right),
$$

so the defining equations for the functions $q_{1}$ and $q_{2}$ can be written either way. There are two main cases here.

Case (a): suppose that $h \in K_{0} \cup K_{1} \cup K_{2} \cup K_{5}$, whence $i_{k} \geq i_{h}^{\prime \prime}$ and $k \in$ $K_{0} \cup K_{1} \cup K_{2} \cup K_{5}$ also. In this case we must have $j_{k}=j_{h}+1$.

Set $\ell^{\prime}=\exp \left(q_{1}(\ell) Z_{1}(\ell)\right) \ell$; since $Z_{2}(\ell)$ is $G$-invariant we have

$$
\begin{aligned}
\sigma^{m}\left(\exp \left(q_{2}(\ell) Z_{2}(\ell)\right) \exp \left(q_{1}(\ell) Z_{1}(\ell)\right) \ell\right) & =\sigma^{m}\left(\exp \left(q_{2}(\ell) Z_{2}(\ell)\right) \ell^{\prime}\right) \\
& =\sigma^{m}\left(\exp \left(q_{2}(\ell) Z_{2}\left(\ell^{\prime}\right)\right) \ell^{\prime}\right) \\
& \left.=\sigma^{m}\left(\exp \left(q_{2}(\ell) \rho_{k-1}\left(Z_{2}\left(\ell^{\prime}\right), \ell^{\prime}\right)\right)\right) \ell^{\prime}\right) \\
& =\sigma^{m}\left(\exp \left(q_{2}(\ell) U_{k}\left(\ell^{\prime}\right)\right) \ell^{\prime}\right) .
\end{aligned}
$$


Put $\sigma_{j}(\ell)=(\sigma(\ell))_{j}$ and $\sigma_{j}^{m}(\ell)=\left(\sigma^{m}(\ell)\right)_{j}$. Now if $j<i_{k}^{\prime}$, then for any $\ell \in \Omega$, $U_{k}(\ell)$ belongs to $\mathfrak{s}_{j}^{\ell}$; in particular, $\left(\exp \left(q_{2}(\ell) U_{k}\left(\ell^{\prime}\right)\right) \ell^{\prime}\right)_{j}=\ell_{j}^{\prime}$. Since $\sigma_{j}^{m}$ depends only upon $\ell_{1}, \ldots, \ell_{j}$, then $\sigma_{j}^{m}\left(\exp \left(q_{2}(\ell) U_{k}\left(\ell^{\prime}\right)\right) \ell^{\prime}\right)=\sigma_{j}^{m}\left(\ell^{\prime}\right)$. We see that $q_{1}(\ell)$ must satisfy

$$
\begin{aligned}
\sigma_{i_{h}}(\ell) & =\sigma_{i_{h}}^{m}\left(\exp \left(q_{2}(\ell) Z_{2}(\ell)\right) \exp \left(q_{1}(\ell) Z_{1}(\ell)\right) \ell\right) \\
& =\sigma_{i_{h}}^{m}\left(\exp \left(q_{2}(\ell) U_{k}\left(\ell^{\prime}\right)\right) \ell^{\prime}\right) \\
& =\sigma_{i_{h}}^{m}\left(\ell^{\prime}\right)=\sigma_{i_{h}}^{m}\left(\exp \left(q_{1}(\ell) Z_{1}(\ell)\right) \ell\right)=\sigma_{i_{h}}^{m}\left(\exp \left(q_{1}(\ell) U_{h}(\ell)\right) \ell .\right.
\end{aligned}
$$

We use the preceding equation to derive an expression for $q_{1}(\ell)$, afterward turning to $q_{2}(\ell)$. The proof branches into the subcases: (a1) $h \in K_{0} \cup K_{1}$, (a2) $h \in K_{2}$, and (a3) $h \in K_{5}$.

Subcase (a1). Setting $j=i_{h}=e_{a}$, we have

$$
\sigma_{j}(\ell)=\zeta_{a}^{\epsilon}(\ell) i \mathfrak{I m}\left(\zeta_{a}^{\epsilon}(\ell)^{-1} \ell_{j}\right)+Y(\ell)
$$

and $\sigma_{j}^{m}(\ell)=\ell_{j}+Y^{m}(\ell)$, where $Y$ and $Y^{m}$ belong to $\mathcal{A}_{j-1}(\Omega)$ and the equation $\sigma_{j}(\ell)=\sigma_{j}^{m}\left(\exp \left(q_{1}(\ell) U_{h}(\ell)\right) \ell\right)$ gives

$$
\ell_{j}-q_{1}(\ell) \ell\left[U_{h}(\ell), Z_{j}\right]=\zeta_{a}^{\epsilon}(\ell) i \mathfrak{I m}\left(\zeta_{a}^{\epsilon}(\ell)^{-1} \ell_{j}\right)+Y(\ell)-Y^{m}(\ell) .
$$

Now Lemma 6.4 applies to obtain the expression for $q_{1}(\ell)$.

Subcase (a2). Set $j=i_{h}-1=e_{a}$. Here $\sigma_{j}(\ell)=0$, while

$$
\sigma_{j}^{m}(\ell)=\zeta_{a}^{m, \epsilon^{m}}(\ell) i \mathfrak{I m}\left(\zeta_{a}^{m, \epsilon^{m}}(\ell)^{-1} \ell_{j}\right)+Y^{m}\left(\ell_{1}, \ldots, \ell_{j-1}\right),
$$

where, as in the proof of Lemma 6.3. Case (b), $O_{\epsilon^{m}}^{m}$ is a covering set for $\Omega^{m}$. Just as in loc. cit. we find that we can replace $\zeta_{a}^{m, \epsilon^{m}}(\ell)$ by $\zeta_{a}^{\epsilon}(\ell)$ so that

$$
\sigma_{j}^{m}(\ell)=\zeta_{a}^{\epsilon}(\ell) i \mathfrak{I m}\left(\zeta_{a}^{\epsilon}(\ell)^{-1} \ell_{j}\right)+Y^{m}(\ell)
$$

and the equation $\sigma_{j}^{m}\left(\exp \left(q_{1}(\ell) U_{h}(\ell)\right) \ell\right)=\sigma_{j}(\ell)$ yields

$$
\zeta_{a}^{\epsilon}(\ell) \mathfrak{I m}\left(\zeta_{a}^{\epsilon}(\ell)^{-1}\left(\ell_{j}-q_{1}(\ell) \ell\left[U_{h}(\ell), Z_{j}\right]\right)\right)=-Y^{m}(\ell) .
$$

Lemma 6.5 applies.

Subcase (a3). Again we set $j=i_{h}-1=e_{a}$ and we have $\sigma_{j}(\ell)=0$. The argument that Lemma 6.6 applies in this case is similar to the preceding, and we omit the details.

Turning to $q_{2}(\ell)$, we have seen that

$$
\sigma^{m}\left(\exp \left(q_{2}(\ell) Z_{2}(\ell)\right) \exp \left(q_{1}(\ell) Z_{1}(\ell)\right) \ell\right)=\sigma^{m}\left(\exp \left(q_{2}(\ell) U_{k}\left(\ell^{\prime}\right)\right) \ell^{\prime}\right),
$$

where $\ell^{\prime}=\exp \left(q_{1}(\ell) Z_{1}(\ell)\right) \ell$. Now the definition of $q_{1}(\ell)$ and $q_{2}(\ell)$ gives

$$
\sigma_{j}^{m}\left(\exp \left(q_{2}(\ell) U_{k}\left(\ell^{\prime}\right)\right) \ell^{\prime}\right)=\sigma_{j}(\ell)=\sigma_{j}\left(\ell^{\prime}\right),
$$

where $j=i_{k}^{\prime}+1$. We then repeat the above argument verbatim, but with $k$ in place of $h$. Thus we find that if $k \in K_{0} \cup K_{1}$, then Lemma 6.4 applies, if $k \in K_{2}$, then Lemma 6.5 applies, while if $k \in K_{5}$, then Lemma 6.6 applies. In each case we get

$$
q_{2}(\ell)=\frac{\ell^{\prime}\left(Z_{i_{k}}\left(\ell^{\prime}\right)\right)}{\ell^{\prime}\left[U_{k}\left(\ell^{\prime}\right), Z_{i_{k}}\left(\ell^{\prime}\right)\right]} \bmod \mathcal{A}_{j-1}(\Omega) .
$$

Now since $G$ is nilpotent in this case, we invoke the invariance of $Z_{i_{k}}(\ell)$ and $\ell\left[U_{k}(\ell), Z_{i_{k}}(\ell)\right]$. Moreover, $\ell_{i}^{\prime}$ depends only upon $\ell_{1}, \ldots, \ell_{j-1}, 1 \leq i \leq j-1$. Hence

$$
q_{2}(\ell)=\frac{\ell^{\prime}\left(Z_{i_{k}}(\ell)\right)}{\ell\left[U_{k}(\ell), Z_{i_{k}}(\ell)\right]} \bmod \mathcal{A}_{j-1}(\Omega) .
$$


Finally note that $q_{1}(\ell)$ depends only upon $\ell_{1}, \ldots, \ell_{j-1}\left(j-1=i_{k}^{\prime}\right.$ now $)$, and it follows that $\ell^{\prime}\left(Z_{i_{k}}(\ell)\right)=\exp \left(q_{1}(\ell) Z_{1}(\ell) \ell\right)\left(Z_{i_{k}}(\ell)\right)=\ell\left(Z_{i_{k}}(\ell)\right) \bmod \mathcal{A}_{j-1}(\Omega)$. Hence the expression

$$
q_{2}(\ell)=\frac{\ell\left(Z_{i_{k}}(\ell)\right)}{\ell\left[U_{k}(\ell), Z_{i_{k}}(\ell)\right]} \bmod \mathcal{A}_{j-1}(\Omega)
$$

is obtained.

Case (b). Here we have $h \in K_{4 a}$ and $k=h+1 \in K_{5 a}$ For ease of notation we set $j=i_{h}, Y=Z_{j}, Y_{1}=\mathfrak{R e} Y, Y_{2}=\mathfrak{I m} Y$, and

$$
c(\ell)=-\beta_{1, k}(\ell)=\frac{\ell\left[U_{h}(\ell), Y_{2}\right]}{\ell\left[U_{h}(\ell), Y_{1}\right]}=\frac{\ell\left[Z_{j}(\ell), \rho_{h-1}\left(Y_{2}, \ell\right)\right]}{\ell\left[Z_{j}(\ell), \rho_{h-1}\left(Y_{1}, \ell\right)\right]},
$$

so that $Z_{i_{k}}(\ell)=Y_{2}-c(\ell) Y_{1}$. Now in this case $\sigma_{j}(\ell)=0$, while $\sigma_{j}^{m}(\ell)=\ell_{j}+Y^{m}(\ell)$ where $Y^{m}(\ell) \in \mathcal{A}_{j-1}(\Omega)$. Thus $q_{1}$ and $q_{2}$ satisfy

$$
\begin{aligned}
\ell_{j}-q_{1}(\ell) \ell\left[U_{h}(\ell), Y\right] & -q_{2}(\ell) \ell\left[U_{k}(\ell), Y\right]+Y^{m}(\ell) \\
& =\sigma_{j}^{m}\left(\exp \left(q_{1}(\ell) U_{h}(\ell)\right) \exp \left(q_{2}(\ell) U_{k}(\ell)\right) \ell\right) \\
& =\sigma_{j}^{m}\left(\exp \left(q_{1}(\ell) Z_{1}(\ell)\right) \exp \left(q_{2}(\ell) Z_{2}(\ell)\right) \ell\right)=0,
\end{aligned}
$$

and since $\ell\left[U_{k}(\ell), Y_{1}\right]=0$, then the preceding equation becomes

$$
\ell(Y)-q_{1}(\ell) \ell\left[U_{h}(\ell), \rho_{h-1}(Y, \ell)\right]-i q_{2}(\ell) \ell\left[U_{k}(\ell), Y_{2}\right]=-Y^{m}\left(\ell_{1}, \ldots, \ell_{j-1}\right) .
$$

Since $q_{1}(\ell) U_{h}(\ell)$ and $q_{2}(\ell) U_{k}(\ell)$ are real, then the real part of the preceding is simply

$$
\ell\left(Y_{1}\right)-q_{1}(\ell) \ell\left[U_{h}(\ell), Y_{1}\right]=\ell\left(Z_{i_{h}}(\ell)\right)-q_{1}(\ell) \ell\left[U_{h}(\ell), Z_{i_{h}}(\ell)\right]=-\mathfrak{R e}\left(Y^{m}(\ell)\right),
$$

while the imaginary part is

$$
\ell\left(Y_{2}\right)-q_{1}(\ell) \ell\left[U_{h}(\ell), Y_{2}\right]-q_{2}(\ell) \ell\left[U_{k}(\ell), Y_{2}\right]=-\mathfrak{I m}\left(Y^{m}(\ell)\right) .
$$

The real part yields the formula for $q_{1}(\ell)$ straightaway. Substituting this into the imaginary part and using the fact that $\ell\left[U_{k}(\ell), Y_{1}\right]=\ell\left[Z_{2}(\ell), V_{h}(\ell)\right]=0$, we obtain

$$
\begin{aligned}
-\mathfrak{I m}\left(Y^{m}(\ell)\right) & =\ell\left(Y_{2}\right)-q_{1}(\ell) \ell\left[U_{h}(\ell), Y_{2}\right]-q_{2}(\ell) \ell\left[U_{k}(\ell), Y_{2}\right] \\
& =\ell\left(Y_{2}\right)-\frac{\ell\left(Y_{1}\right)+\mathfrak{R} \mathfrak{e}\left(Y^{m}(\ell)\right)}{\ell\left[U_{h}(\ell), Y_{1}\right]} \ell\left[U_{h}(\ell), Y_{2}\right]-q_{2}(\ell) \ell\left[U_{k}(\ell), Y_{2}\right] \\
& =\ell\left(Y_{2}-c(\ell) Y_{1}\right)-q_{2}(\ell) \ell\left[U_{k}(\ell), Y_{2}-c(\ell) Y_{1}\right] \bmod \mathcal{A}_{j-1}(\Omega) \\
& =\ell\left(Z_{i_{k}}(\ell)\right)-q_{2}(\ell) \ell\left[U_{k}(\ell), Z_{i_{k}}(\ell)\right] \bmod \mathcal{A}_{j-1}(\Omega),
\end{aligned}
$$

from which we obtain the expression for $q_{2}(\ell)$. This completes the proof.

\section{CANONICAL COORDINATES}

For any open set $U$ of $\mathfrak{g}^{*}$ let $\mathcal{E}(U)$ be the space of all smooth complex valued functions on $U$ and $\mathcal{V}(U)$ the space of all smooth vector fields on $U$. For $\phi \in \mathcal{E}(U)$ and $\ell \in U$, let $X_{\ell}^{\phi}$ be the element of $\mathfrak{s}$ identified with $d \phi(\ell)$. Each function $\phi \in \mathcal{E}(U)$ gives rise to the Hamiltonian vector field $\xi^{\phi}$ defined on $U$ by $\xi_{\ell}^{\phi}=\xi_{\ell}^{X_{\ell}^{\phi}}$. Recall that if $\psi_{X}$ denotes the coordinate function $\ell \mapsto \ell(X)$ on $\mathfrak{g}^{*}$ for $X \in \mathfrak{s}$, then $\xi^{\psi_{X}}=\xi^{X}$ as defined in Section 1 . 
The Poisson bracket on $\mathcal{E}(U)$ is defined by

$$
\{\phi, \psi\}(\ell)=\ell\left[X_{\ell}^{\phi}, X_{\ell}^{\psi}\right], \quad \ell \in U,
$$

and one has $\xi^{\{\phi, \psi\}}=\left[\xi^{\phi}, \xi^{\psi}\right]$. Note that if $\mathcal{O}$ is a coadjoint orbit contained in $U$ and $\left.\phi\right|_{\mathcal{O}}=\left.\phi^{\prime}\right|_{\mathcal{O}}$, then for each $\ell \in \mathcal{O}$, their differentials agree on $T_{\ell}(\mathcal{O})$, and it follows that their Hamiltonians agree at each $\ell \in \mathcal{O}$.

We fix, as we have throughout, an adaptable basis $\left\{Z_{1}, Z_{2}, \ldots, Z_{n}\right\}$ for $\mathfrak{s}$ and a corresponding ultrafine layer $\Omega$ in $\mathfrak{g}^{*}$. The layer $\Omega$ may not be a submanifold of $\mathfrak{g}^{*}$, but we shall say that a complex-valued function $\phi$ on $\Omega$ is smooth if there is an open set $U$ with $\Omega \subset U$ and a function $\tilde{\phi} \in \mathcal{E}(U)$ such that $\phi=\left.\tilde{\phi}\right|_{\Omega}$. Denote by $\mathcal{E}(\Omega)$ the algebra of all smooth functions on $\Omega$; observe that $\mathcal{E}(\Omega)=\tilde{\mathcal{E}}(\Omega) / \sim$, where $\tilde{\mathcal{E}}(\Omega)$ is the set of all pairs $(\tilde{\phi}, U)$ where $U$ is an open set containing $\Omega$ and $\tilde{\phi} \in \mathcal{E}(U)$, and where $\left(\phi_{1}, U_{1}\right) \sim\left(\phi_{2}, U_{2}\right)$ if and only if $\left.\phi_{1}\right|_{\Omega}=\left.\phi_{2}\right|_{\Omega}$. It follows from the introductory remarks above that the Poisson bracket is well defined on $\mathcal{E}(\Omega)$ in the obvious way. We say that a vector field $\xi$ on $\Omega$ is smooth if $\xi(\mathcal{E}(\Omega)) \subset \mathcal{E}(\Omega)$. We denote by $\mathcal{V}(\Omega)$ the Lie algebra of smooth vector fields on $\Omega$.

Recall that we have the complex Vergne polarizations $\mathfrak{h}(\ell), \ell \in \Omega$, naturally associated with the layer $\Omega$. In particular, the mapping $\ell \mapsto \mathfrak{h}(\ell)$ is rational in a sense that is evident. For each $\ell \in \Omega$, put

$$
\mathcal{F}(\ell)=\left\{\xi_{\ell}^{Y} \mid Y \in \mathfrak{h}(\ell)\right\} \subset T_{\ell}\left(\mathfrak{g}^{*}\right)_{\mathbb{C}} .
$$

Since $s \mathfrak{h}(\ell)=\mathfrak{h}(s \ell)$ holds for all $\ell \in \Omega, s \in G$, it follows that for each coadjoint orbit $\mathcal{O}$ in $\Omega,\left.\mathcal{F}\right|_{\mathcal{O}}$ is a $G$-invariant complex (geometric) polarization of the symplectic manifold $\left(\mathcal{O}, \omega_{\mathcal{O}}\right)$.

Set

$$
\mathcal{V}^{0}(\Omega)=\left\{\xi \in \mathcal{V}(\Omega) \mid \xi_{\ell} \in \mathcal{F}(\ell) \text { holds for all } \ell \in \Omega\right\}
$$

and

$$
\mathcal{E}^{0}(\Omega)=\left\{\phi \in \mathcal{E}(\Omega) \mid \xi^{\phi} \in \mathcal{V}^{0}(\Omega)\right\} .
$$

For any orbit $\mathcal{O}$ in $\Omega$, we define $\mathcal{V}^{0}(\mathcal{O})$ as in [6], and it is clear that if $\xi \in \mathcal{V}(\Omega)$, then $\xi \in \mathcal{V}^{0}(\Omega)$ if and only $\xi \in \mathcal{V}^{0}(\mathcal{O})$ for all $\mathcal{O} \subset \Omega$. Similarly for any $\phi \in \mathcal{E}(\Omega)$, we have $\phi \in \mathcal{E}^{0}(\Omega)$ if and only if $\phi \in \mathcal{E}^{0}(\mathcal{O})$ holds for all orbits $\mathcal{O}$ in $\Omega$. We also have the following.

Proposition 7.1. One has

$$
\mathcal{E}^{0}(\Omega)=\left\{\phi \in \mathcal{E}(\Omega) \mid \xi(\phi)=0 \text { holds for all } \xi \in \mathcal{V}^{0}(\Omega)\right\} .
$$

Moreover, $\mathcal{E}^{0}(\Omega)$ is a maximal abelian Lie subalgebra of $\mathcal{E}(\Omega)$.

Proof. Let $\xi \in \mathcal{V}^{0}(\Omega)$. For each $\ell \in \Omega, \xi_{\ell} \in \mathcal{F}(\ell)$, and so we have $Y_{\ell} \in \mathfrak{h}(\ell)$ such that $\xi_{\ell}=\ell\left[Y_{\ell}, \cdot\right]$. Now given $\phi \in \mathcal{E}(\Omega)$, we have $\phi \in \mathcal{E}^{0}(\Omega)$ if and only if $X_{\ell}^{\phi} \in \mathfrak{h}(\ell)$ holds for all $\ell \in \Omega$. Hence $\phi \in \mathcal{E}^{0}(\Omega)$ if and only if $\xi_{\ell}(\phi)=d \phi(\ell)\left(\xi_{\ell}\right)=\ell\left[Y_{\ell}, X_{\ell}^{\phi}\right]=0$ holds for all $\ell \in \Omega$.

To verify the second assertion, let $\phi \in \mathcal{E}(\Omega)$ and suppose that for all $\psi \in \mathcal{E}^{0}(\Omega)$, $\{\phi, \psi\}=0$. For each $\ell \in \Omega$ and $Y_{\ell} \in \mathfrak{h}(\ell)$, we have $0=\left\{\phi, \psi_{Y_{\ell}}\right\}(\ell)=\ell\left[X_{\ell}^{\phi}, Y_{\ell}\right]$ so that $X_{\ell}^{\phi} \in \mathfrak{h}(\ell)$ for all $\ell \in \Omega$, and hence $\phi \in \mathcal{E}^{0}(\Omega)$.

We now define what we mean by a system $\left(p_{1}, \ldots, p_{d}, q_{1}, \ldots, q_{d}\right)$ of adaptable coordinates for $\Omega$. Write the subindices $K=\{1,2, \ldots, d\}$ as $\left\{k_{1}, k_{2}, \ldots, k_{d}\right\}$ so that for values $j_{k}$ of the sequence $\mathbf{j}$, we have $j_{k_{r}}<j_{k_{r+1}}, 1 \leq r \leq d$. Write $\left\{1 \leq r \leq d \mid k_{r} \in K_{3}\right\}=\left\{r_{1}<\cdots<r_{b}\right\}$ and set $E=\mathbb{R}^{2 d-2 b} \times \mathbb{C}^{b}$. 
Definition 7 (Adaptable coordinates). We will call a system of functions $\left(p_{1}, \ldots\right.$, $\left.p_{d}, q_{1}, \ldots, q_{d}\right)$ adaptable coordinates for $\Omega$ if they satisfy the following:

(a) $p_{r}$ and $q_{r}$ belong to $\mathcal{A}(\Omega), 1 \leq r \leq d$.

(b) One has $q_{r} \in \mathcal{E}^{0}(\Omega), 1 \leq r \leq d$.

(c) The two-form

$$
\omega=\sum_{r=1}^{d} d p_{r} \wedge d q_{r}
$$

satisfies

$$
\omega_{\ell}\left(\xi_{\ell}^{X}, \xi_{\ell}^{Y}\right)=\ell[X, Y]
$$

for all $X, Y \in \mathfrak{s}, \ell \in \Omega$.

(d) There is a covering of $\Omega$ by $G$-invariant open sets $U_{\epsilon}$, each of which has the following. For each $k_{r} \notin K_{3}$, there is a $\mathbb{T}$-valued $G$-invariant analytic function $\tau_{r}^{\epsilon}$ on $U_{\epsilon}$ such that $v_{r}^{\epsilon}(\ell)=\tau_{r}^{\epsilon}(\ell) q_{r}(\ell)$ and $u_{r}^{\epsilon}(\ell)=\tau_{r}^{\epsilon}(\ell)^{-1} p_{r}(\ell)$ are real valued, and so that the mapping $c^{\epsilon}: U_{\epsilon} \rightarrow E$ defined by

$$
c^{\epsilon}(\ell)=\left(u^{\epsilon}(\ell), v^{\epsilon}(\ell), q_{r_{1}}(\ell), \ldots, q_{r_{b}}(\ell)\right)
$$

restricts to a diffeomorphism of each orbit $\mathcal{O}$ in $U_{\epsilon}$ onto $E$ (here $u^{\epsilon}(\ell)=$ $\left(u_{r}^{\epsilon}(\ell)\right)_{r, k_{r} \notin K_{3}}$ and $\left.v^{\epsilon}(\ell)=\left(v_{r}^{\epsilon}(\ell)\right)_{r, k_{r} \notin K_{3}}\right)$. Moreover, for $r \in K_{3}$, there is a real-valued $G$-invariant function $\tau_{r}$ on $\Omega$ such that the function $p_{r}$ is of the form $p_{r}(\ell)=i \tau_{r}(\ell) \overline{q_{r}}(\ell)$.

In the next result we give an explicit construction of adaptable coordinates for the layer $\Omega$.

Theorem 7.2 (Canonical coordinates). Let $\mathfrak{g}$ be an exponential Lie algebra and fix an adaptable basis for the complexification $\mathfrak{s}$ of $\mathfrak{g}$. Let $\Omega$ be an ultrafine layer with its cross-section $\Sigma$. Then there is an explicit construction for a system of adaptable coordinates for $\Omega$.

Proof. Let $m=n^{\prime}$ and $\Omega^{\circ}, \Sigma^{\circ}$ be the ultrafine layer and cross-section in $\mathfrak{g}_{m}^{*}$ lying below $\Omega, \Sigma$.

Recall that we have the natural embedding $\pi^{*}: \phi \mapsto \phi \circ \pi$ of $\mathcal{E}\left(\mathfrak{g}_{m}^{*}\right)$ into $\mathcal{E}\left(\mathfrak{g}^{*}\right)$, and that for convenience the notation $\phi(\ell)=\phi\left(\ell_{1}, \ldots, \ell_{m}\right)$ will occasionally be used both for $\phi \in \mathcal{E}\left(\mathfrak{g}_{m}^{*}\right)$ and $\pi^{*} \phi$. Also, by means of our $\mathfrak{g}^{*}$-coordinates we regard $\mathfrak{g}_{m}^{*}$ and $\mathfrak{s}_{m}^{*}$ as subspaces of $\mathfrak{g}^{*}$ and $\mathfrak{s}^{*}$, respectively, and with the identifications of $T_{\ell}\left(\mathfrak{g}^{*}\right)$ with $\mathfrak{g}^{*}$ and $T_{\pi(\ell)}\left(\mathfrak{g}_{m}^{*}\right)$ with $\mathfrak{g}_{m}^{*}$, obtain the corresponding inclusion of tangent spaces. Since $\pi(\Omega) \subset \Omega^{\circ}$, we have natural embeddings $\pi^{*}: \mathcal{E}\left(\Omega^{\circ}\right) \hookrightarrow \mathcal{E}(\Omega)$ and $\pi^{*}: \mathcal{V}\left(\Omega^{\circ}\right) \hookrightarrow \mathcal{V}(\Omega)$. Further, for $\phi \in \mathcal{E}\left(\Omega^{\circ}\right)$, one has $X_{\ell}^{\phi \circ \pi}=d(\phi \circ \pi)(\ell)=$ $d \phi(\pi(\ell))=X_{\pi(\ell)}^{\phi}$, so denoting by $\xi\left(\xi^{\circ}\right)$ the homomorphism $\phi \mapsto \xi^{\phi}$ of $\mathcal{E}(\Omega)\left(\mathcal{E}\left(\Omega^{\circ}\right)\right)$ into $\mathcal{V}(\Omega)\left(\mathcal{V}\left(\Omega^{\circ}\right)\right)$, we have $\pi^{*} \circ \xi^{\circ}=\xi \circ \pi^{*}$.

Let $2 d^{\circ}$ be the dimension of orbits in $\Omega^{\circ}$. We assume that $d^{\circ}>0$, the proof in the case where $d^{\circ}=0$ is a simplification of what follows. Suppose that $\left(p_{1}^{\circ}, \ldots, p_{d^{\circ}}^{\circ}\right.$, $q_{1}^{\circ}, \ldots, q_{d^{\circ}}^{\circ}$ is a system of adaptable coordinates for $\Omega^{\circ}$, and denote by $c^{\circ}$ the corresponding global chart so that for each $G_{m}$-orbit $\mathcal{O}^{\circ}$ in $\Omega^{\circ}, c^{\circ}$ is a diffeomorphism of $\mathcal{O}^{\circ}$ onto $E^{\circ}=\mathbb{R}^{2 d^{\circ}-2 b^{\circ}} \times \mathbb{C}^{b^{\circ}}$. For $\ell \in \Omega$, let $\mathfrak{h}(\pi(\ell))=\mathfrak{h}(\ell) \cap \mathfrak{s}_{m}$ be the Vergne polarization at $\pi(\ell)$ with respect to the adaptable basis $\left\{Z_{1}, Z_{2}, \ldots, Z_{m}\right\}$ for $\mathfrak{s}_{m}$.

Case 0: suppose that $\mathbf{n} \cap \mathbf{e}=\emptyset$, so that $d^{\circ}=d$ and $b^{\circ}=b$. In this case both $\mathfrak{h}(\pi(\ell))+\mathfrak{s}_{\ell}=\mathfrak{h}(\ell)$ and $\mathfrak{s}(\ell) \cap \mathfrak{s}_{m}=\mathfrak{s}_{m}(\pi(\ell))$ hold for each $\ell$, and hence $\mathfrak{h}(\ell) / \mathfrak{s}(\ell)$ is isomorphic with $\mathfrak{h}(\pi(\ell)) / \mathfrak{s}_{m}(\pi(\ell))$. It follows that $\left.d \pi\right|_{\mathcal{F}(\ell)}$ is an isomorphism of 
$\mathcal{F}(\ell)$ onto $\mathcal{F}(\pi(\ell))$, and $\mathcal{F}(\ell)=\left\{\mu \in \mathfrak{s}^{*} \mid d \pi(\mu) \in \mathcal{F}(\pi(\ell))\right\}$. It is clear from this and the remarks preceding this theorem that we may regard $\mathcal{E}^{0}\left(\Omega^{\circ}\right)$ as a subspace of $\mathcal{E}^{0}(\Omega)$ in this case.

Now define $p_{r}=\pi^{*} p_{r}^{\circ}$ and $q_{r}=\pi^{*} q_{r}^{\circ}, 1 \leq r \leq d$. We have $q_{r} \in \mathcal{E}^{0}(\Omega)$ for all $r$. Fix $\ell \in \Omega$; given $X, Y \in \mathfrak{s}$, write $X=X(\ell)+S(\ell), Y=Y(\ell)+T(\ell)$, where $S(\ell)$, $T(\ell) \in \mathfrak{s}(\ell)$. Then

$$
\begin{aligned}
\sum d p_{r}(\ell) \wedge d q_{r}(\ell)\left(\xi_{\ell}^{X}, \xi_{\ell}^{Y}\right) & =\sum d p_{r}^{\circ}(\pi(\ell)) \wedge d q_{r}^{\circ}(\pi(\ell))\left(\xi_{\pi(\ell)}^{X(\ell)}, \xi_{\pi(\ell)}^{Y(\ell)}\right) \\
& =\pi(\ell)[X(\ell), Y(\ell)]=\ell[X, Y] .
\end{aligned}
$$

The chart $c$ is obtained from the chart $c^{\circ}$ by defining $\tau_{r}^{\epsilon}=\pi^{*} \tau_{r}^{\epsilon^{\circ}}$.

Case 1: Suppose that $\mathbf{n} \cap \mathbf{e}=\{j\}$ with $j$ a value of $\mathbf{j}$. Here we have $d^{\circ}=d-1$ and $b^{\circ}=b$. Let $q_{d}(\ell)=q(\ell)$ as defined in Proposition 6.1 and define

$$
p_{d}(\ell)=\ell\left(Z_{j}(\ell)\right) \text {. }
$$

We observe the following:

i) For each $\ell \in \Omega, \xi_{\ell}^{X} p_{d}=\ell\left[X, Z_{j}(\ell)\right]$ holds for all $X \in \mathfrak{s}$.

ii) The function $q_{d}$ is $\mathfrak{s}_{m}$-invariant.

iii) For each $\ell \in \Omega, \xi_{\ell}^{Z_{j}(\ell)} q_{d}=1$.

Since $\mathfrak{h}(\ell)=\mathfrak{h}(\ell) \cap \mathfrak{s}_{m}+\mathfrak{s}(\ell)$, we have $\mathcal{F}(\ell)=\left\{\xi_{\ell}^{Y} \mid Y \in \mathfrak{h}(\ell) \cap \mathfrak{s}_{m}\right\}$, and so it follows from this and the preceding observation ii) that $q_{d}$ belongs to $\mathcal{E}^{0}(\Omega)$. For $1 \leq r \leq d-1$, define $q_{r}$ and $p_{r}$ by

$$
q_{r}(\ell)=q_{r}^{\circ}\left(\exp \left(q_{d}(\ell) Z_{j}(\ell)\right) \ell\right), \quad p_{r}(\ell)=p_{r}^{\circ}\left(\exp \left(q_{d}(\ell) Z_{j}(\ell)\right) \ell\right) .
$$

Then we have

$$
\xi_{\ell}^{Z_{j}(\ell)} q_{r}=\xi_{\ell}^{Z_{j}(\ell)} p_{r}=0, \quad 1 \leq r \leq d-1 .
$$

We claim that the functions $q_{r}, 1 \leq r \leq d-1$, belong to $\mathcal{E}^{0}(\Omega)$. Fix $\ell \in \Omega$, put $\ell^{\prime}=\exp \left(q_{d}(\ell) Z_{j}(\ell)\right) \ell$, and take $Y \in \mathfrak{h}(\ell)$. First, suppose $Y \in \mathfrak{g}_{m}$ and set $Y^{\prime}=\exp \left(q_{d}(\ell) Z_{j}(\ell)\right) Y$; then $Y^{\prime} \in \mathfrak{h}\left(\ell^{\prime}\right)$ and hence

$$
\xi_{\ell^{\prime}}^{Y^{\prime}} q_{r}^{\circ}=0 .
$$

But since $Z_{j}(\ell)$ is $G_{m}$-invariant (Corollary 3.1 ) and $q_{d}(\ell)$ also (by definition), it follows that

$$
\xi_{\ell^{\prime}}^{Y^{\prime}} q_{r}^{\circ}=\xi_{\ell}^{Y} q_{r}=0
$$

Now if $Y=U+i V\left(U\right.$ and $V$ in $\left.\mathfrak{g}_{m}\right)$, the same computation gives

$$
\xi_{\ell^{\prime}}^{Y^{\prime}} q_{r}^{\circ}=\xi_{\ell^{\prime}}^{U^{\prime}} q_{r}^{\circ}+i \xi_{\ell^{\prime}}^{V^{\prime}} q_{r}^{\circ}=\xi_{\ell}^{Y} q_{r}=0
$$

and the claim is proved. Defining $\omega_{d-1}=\sum_{r=1}^{d-1} d p_{r} \wedge d q_{r}$, by $G_{m}$-invariance of $Z_{j}(\ell)$ and $q_{d}(\ell)$, it follows that $\left(\omega_{d-1}\right)_{\ell}\left(\xi_{\ell}^{X}, \xi_{\ell}^{Y}\right)=\ell[X, Y]$ holds for all $X, Y \in \mathfrak{s}_{m}$, $\ell \in \Omega$. Now set

$$
\omega=\sum_{k=1}^{d} d p_{r} \wedge d q_{r}
$$

fix $\ell \in \Omega$, and let $X, Y \in \mathfrak{s}$. If $X, Y \in \mathfrak{s}_{m}$, then $\mathfrak{s}_{m}$-invariance of $q_{d}$ gives

$$
\omega_{\ell}\left(\xi_{\ell}^{X}, \xi_{\ell}^{Y}\right)=\left(\omega_{d-1}\right)_{\ell}\left(\xi_{\ell}^{X}, \xi_{\ell}^{Y}\right)=\ell[X, Y]
$$


Suppose $X=Z_{j}(\ell)$ and $Y \in \mathfrak{s}_{m}$. By equation (7.1) we have

$$
\begin{aligned}
\omega_{\ell}\left(\xi_{\ell}^{X}, \xi_{\ell}^{Y}\right) & =\left(d p_{d} \wedge d q_{d}\right)_{\ell}\left(\xi_{\ell}^{X}, \xi_{\ell}^{Y}\right)=-\xi_{\ell}^{X} q_{d} \cdot \xi_{\ell}^{Y} p_{d} \\
& =-\xi_{\ell}^{Y} p_{d}=\ell[X, Y] .
\end{aligned}
$$

Finally we show that the functions $p_{r}$ and $q_{r}$ constructed above satisfy the last condition for adaptable coordinates. If $\mathfrak{g}$ is nilpotent, then set $U_{\epsilon}=O_{\epsilon}$ and $\tau_{d}^{\epsilon}(\ell)=$ $\phi_{j}^{\epsilon}(\ell)$. Note that in this case Lemma 3.3 shows that $\tau_{d}^{\epsilon}(\ell)$ is $G$-invariant. If $\mathfrak{g}$ is not nilpotent, then $m=n-1, Z_{j}(\ell)=Z_{j}=Z_{n} \in \mathfrak{g}$ and $q(\ell)$ is real-valued. We set

$$
U_{\epsilon}=\left\{\ell \in \Omega \mid \exp \left(q(\ell) Z_{n}\right) \ell \in U_{\epsilon^{\circ}}\right\}
$$

and $\tau_{d}^{\epsilon}(\ell)=1$. In any case, for $1 \leq r \leq d-1$ with $k_{r} \notin K_{3}$, put

$$
\tau_{r}^{\epsilon}(\ell)=\tau_{r}^{\epsilon^{\circ}}\left(\exp \left(q(\ell) Z_{j}(\ell)\right) \ell\right) .
$$

We claim that for any $G_{m^{-}}$-invariant function $\eta^{\circ} \in \mathcal{E}\left(U_{\epsilon^{\circ}}\right)$, the function $\eta(\ell)=$ $\eta^{\circ}\left(\exp \left(q_{d}(\ell) Z_{j}(\ell)\right) \ell\right)$ is $G$-invariant. Set $X(\ell)=\phi_{j}(\ell)^{-1} Z_{j}(\ell)$ so that $X(\ell) \in \mathfrak{g}$; we observe that if $\ell^{\prime}=\exp (t X(\ell)) s \ell$ where $s \in G_{m}$, then $q_{d}\left(\ell^{\prime}\right)=q_{d}(\ell)-t \phi_{j}(\ell)^{-1}$, and since $Z_{j}(\ell)$ is $G$-invariant,

$$
\begin{aligned}
\eta\left(\ell^{\prime}\right) & =\eta^{\circ}\left(\exp \left(q_{d}\left(\ell^{\prime}\right) Z_{j}(\ell)\right) \ell^{\prime}\right) \\
& =\eta^{\circ}\left(\exp \left(q_{d}(\ell) Z_{j}(\ell)\right) \exp (-t X(\ell)) \exp (t X(\ell)) s \ell\right) \\
& =\eta^{\circ}\left(s^{\prime} \exp \left(q_{d}(\ell) Z_{j}(\ell)\right) \ell\right) \\
& =\eta^{\circ}\left(\exp \left(q_{d}(\ell) Z_{j}(\ell)\right) \ell\right)=\eta(\ell),
\end{aligned}
$$

proving the claim. Hence from the preceding claim and the induction hypothesis, we have that $\tau_{r}^{\epsilon}(\ell)$ is $G$-invariant for $r<d$. Now it is clear that for each $r, \tau_{r}^{\epsilon}(\ell) q_{r}(\ell)$ and $\tau_{r}^{\epsilon}(\ell) p_{r}(\ell)$ are real-valued analytic functions on $U_{\epsilon}$, and it remains to show that the map $c$ is bijective on each orbit $\mathcal{O}$.

Let $\ell, \ell^{\prime} \in \mathcal{O} \subset U_{\epsilon}$ with $\ell \neq \ell^{\prime}$. If $\pi(\ell)=\pi\left(\ell^{\prime}\right)$, then the fact that $\mathfrak{s}=$ $\mathbb{C} Z_{j}(\ell)+\mathfrak{s}(\ell)+\mathfrak{s}_{m}$ implies $p_{d}(\ell) \neq p_{d}\left(\ell^{\prime}\right)$, and hence $u_{d}(\ell) \neq u_{d}\left(\ell^{\prime}\right)$. Suppose that $\pi(\ell) \neq \pi\left(\ell^{\prime}\right)$. If $\pi(\ell)$ and $\pi\left(\ell^{\prime}\right)$ belong to the same $G_{m}$-orbit, then by definition of $q_{d}$ we have $q_{d}(\ell)=q_{d}\left(\ell^{\prime}\right)$. By induction we have $c^{\circ}(\ell) \neq c^{\circ}\left(\ell^{\prime}\right)$, and it follows that $c(\ell) \neq c\left(\ell^{\prime}\right)$. If $\pi(\ell)$ and $\pi\left(\ell^{\prime}\right)$ do not belong to the same $G_{m}$-orbit, then by definition of $q_{d}(\ell)$ we have $q_{d}(\ell) \neq q_{d}\left(\ell^{\prime}\right)$, and hence $v_{d}(\ell) \neq v_{d}\left(\ell^{\prime}\right)$ so that $c(\ell) \neq c\left(\ell^{\prime}\right)$.

Finally, let $e \in E$ and write $e=\left(e^{\circ}, x, y\right)$, where $e^{\circ} \in E^{\circ}$ and $x, y \in \mathbb{R}$. The relation $q_{d}(\exp (t X(\ell)) \ell)=q_{d}(\ell)-t \phi_{j}(\ell)^{-1}$ shows that we can choose $\ell \in \Omega$ such that $v_{d}(\ell)=y$. By induction $c^{\circ}$ maps $G_{m} \pi(\ell)$ onto $E^{\circ}$, so we choose $s \in G_{m}$ such that $\ell^{\prime}=s \ell$ satisfies $c^{\circ}\left(\ell^{\prime}\right)=e^{\circ}$. By $G_{m}$-invariance of $q_{d}$ we still have $v_{d}\left(\ell^{\prime}\right)=y$. Choosing $Y\left(\ell^{\prime}\right) \in \mathfrak{g}_{m}\left(\ell^{\prime}\right) \backslash \mathfrak{g}\left(\ell^{\prime}\right)$ (for example $Y\left(\ell^{\prime}\right)=\phi_{i_{k}}\left(\ell^{\prime}\right)^{-1} V_{k}\left(\ell^{\prime}\right)$, where $k$ is the subindex for which $j=j_{k}$ ) we see that there is $t \in \mathbb{R}$ such that $\ell^{\prime \prime}=\exp \left(t Y\left(\ell^{\prime}\right)\right) \ell^{\prime}$ satisfies $u_{d}\left(\ell^{\prime \prime}\right)=x$, as well as $v_{d}\left(\ell^{\prime \prime}\right)=y$ and $c^{\circ}\left(\ell^{\prime \prime}\right)=e^{\circ}$. Thus $c$ is surjective.

Case 2: Suppose that $\mathbf{n} \cap \mathbf{e}$ consists of two values $j_{h}$ and $j_{k}$ of the sequence $\mathbf{j}$, with $h<k$. Here we have $d^{\circ}=d-2$ and $b^{\circ}=b$. Let $q_{(1)}$ and $q_{(2)}$ be the functions defined in Proposition 6.1 recall that these functions are defined uniquely by

$$
\pi\left(\exp \left(q_{(1)}(\ell) Z_{1}(\ell)\right) \exp \left(q_{(2)}(\ell) Z_{2}(\ell)\right) \ell\right) \in \mathcal{O}_{\pi(\sigma(\ell))},
$$

where

$$
Z_{1}(\ell)=Z_{j_{h}}(\ell), \quad Z_{2}(\ell)=Z_{j_{k}}(\ell)
$$


Define $q_{d-1}^{1}(\ell)=q_{(1)}(\ell)$ and

$$
p_{d-1}^{1}(\ell)=\ell\left(Z_{1}(\ell)\right) .
$$

Then just as in Case 1, we have the following:

i) For any $X \in \mathfrak{s}, \xi_{\ell}^{X} p_{d-1}^{1}=\ell\left[X, Z_{1}(\ell)\right]$.

ii) $q_{d-1}^{1}$ (in fact both $q_{(1)}$ and $q_{(2)}$ ) are $\mathfrak{s}_{m}$-invariant.

iii) $\xi_{\ell}^{Z_{1}(\ell)} q_{(1)}=1$.

Observe that in this case $\mathfrak{h}(\ell) \subset \mathfrak{s}_{m}$, so that $q_{(1)}$ and $q_{(2)}$ belong to $\mathcal{E}^{0}(\Omega)$. Define $p_{r}^{1}, q_{r}^{1}, r=1, \ldots, d-2$, by

$$
p_{r}^{1}(\ell)=p_{r}^{\circ}\left(\exp \left(q_{(1)}(\ell) Z_{1}(\ell)\right) \ell\right), \quad q_{r}^{1}(\ell)=q_{r}^{\circ}\left(\exp \left(q_{(1)}(\ell) Z_{1}(\ell)\right) \ell\right) .
$$

We have

$$
\xi_{\ell}^{Z_{1}(\ell)} q_{r}^{1}=\xi_{\ell}^{Z_{1}(\ell)} p_{r}^{1}=0, \quad 1 \leq r \leq d-2 .
$$

Following verbatim the argument of Case 1, we find that $q_{r}^{1}$ belongs to $\mathcal{E}^{0}(\Omega)$, $1 \leq r \leq d-2$. Setting

$$
\omega_{d-2}^{1}=\sum_{r=1}^{d-2} d p_{r}^{1} \wedge d q_{r}^{1},
$$

we have

$$
\left(\omega_{d-2}^{1}\right)_{\ell}\left(\xi_{\ell}^{X}, \xi_{\ell}^{Y}\right)=\ell[X, Y], \quad X, Y \in \mathfrak{s}_{m}
$$

Define

$$
\omega_{d-1}^{1}=\sum_{r=1}^{d-1} d p_{r}^{1} \wedge d q_{r}^{1}
$$

and put $\mathfrak{s}^{\mathcal{O}}=\mathfrak{s}_{m}+\mathbb{C} Z_{1}(\ell)$. We repeat the argument given in Case 1 for $\omega$ to show that for each $\ell \in \Omega$ and $X, Y \in \mathfrak{s}^{\mathcal{O}}, \omega^{1}\left(\xi_{\ell}^{X}, \xi_{\ell}^{Y}\right)=\ell[X, Y]$ holds. First suppose that $X, Y \in \mathfrak{s}_{m} ;$ then

$$
\left(\omega_{d-1}^{1}\right)_{\ell}\left(\xi_{\ell}^{X}, \xi_{\ell}^{Y}\right)=\left(\omega_{d-2}^{1}\right)_{\ell}\left(\xi_{\ell}^{X}, \xi_{\ell}^{Y}\right)=\ell[X, Y],
$$

because $q_{d-1}^{1}$ is $\mathfrak{s}_{m}$-invariant. Next suppose $X=Z_{1}(\ell)$ and $Y \in \mathfrak{s}_{m}$; then

$$
\begin{aligned}
\left(\omega_{d-1}^{1}\right)_{\ell}\left(\xi_{\ell}^{X}, \xi_{\ell}^{Y}\right) & =\left(d p_{d-1}^{1} \wedge d q_{d-1}^{1}\right)_{\ell}\left(\xi_{\ell}^{X}, \xi_{\ell}^{Y}\right)=-\xi_{\ell}^{X} q_{d-1}^{1} \xi_{\ell}^{Y} p_{d-1}^{1} \\
& =-\xi_{\ell}^{Y} p_{d-1}^{1}=\ell[X, Y] .
\end{aligned}
$$

Now define $q_{r}, p_{r}$ for $r=1, \ldots, d-1$, by the following:

$$
q_{r}(\ell)=q_{r}^{1}\left(\exp \left(q_{(2)}(\ell) Z_{2}(\ell)\right) \ell\right), \quad p_{r}(\ell)=p_{r}^{1}\left(\exp \left(q_{(2)}(\ell) Z_{2}(\ell)\right) \ell\right) .
$$

Recall that in the proof of Proposition 6.4 we observed that

$$
\begin{aligned}
& \sigma^{m}\left(\exp \left(q_{(1)}(\ell) Z_{1}(\ell)\right) \exp \left(q_{(2)}(\ell) Z_{2}(\ell)\right) \ell\right) \\
& \quad=\sigma^{m}\left(\exp \left(q_{(2)}(\ell) Z_{2}(\ell)\right) \exp \left(q_{(1)}(\ell) Z_{1}(\ell)\right) \ell\right)
\end{aligned}
$$

from which it follows that $q_{(1)}\left(\exp \left(q_{(2)}(\ell) Z_{2}(\ell) \ell\right)=q_{(1)}(\ell)\right.$; thus we still have $q_{d-1}=$ $q_{(1)}$. We have

$$
\xi_{\ell}^{Z_{2}(\ell)} q_{r}=\xi_{\ell}^{Z_{2}(\ell)} p_{r}=0, \quad 1 \leq r \leq d-1 .
$$

Now set $q_{d}=q_{(2)}$ and $p_{d}(\ell)=\ell\left(Z_{2}(\ell)\right), \ell \in \Omega$. We have

i) for $X \in \mathfrak{s}, \xi_{\ell}^{X} p_{d}=\ell\left[X, Z_{2}(\ell)\right]$,

ii) $\xi_{\ell}^{q_{(1)}(\ell) Z_{1}(\ell)} q_{d}=0$, and hence $q_{d}$ is $\mathfrak{s}^{\mathcal{O}}$-invariant,

iii) $\xi_{\ell}^{Z_{2}(\ell)} q_{d}=1$. 
Set

$$
\omega_{d-1}=\sum_{r=1}^{d-1} d p_{r} \wedge d q_{r}, \quad \omega=\sum_{k=1}^{d} d p_{r} \wedge d q_{r} .
$$

By again repeating arguments given above we obtain

$$
\left(\omega_{d-1}\right)_{\ell}\left(\xi_{\ell}^{X}, \xi_{\ell}^{Y}\right)=\ell[X, Y], \quad \forall X, Y \in \mathfrak{s}^{\mathcal{O}},
$$

and hence for $X, Y \in \mathfrak{s}^{\mathcal{O}}$,

$$
(\omega)_{\ell}\left(\xi_{\ell}^{X}, \xi_{\ell}^{Y}\right)=\left(\omega_{d-1}\right)_{\ell}\left(\xi_{\ell}^{X}, \xi_{\ell}^{Y}\right)=\ell[X, Y]
$$

while, if $X=Z_{2}(\ell), Y \in \mathfrak{s}^{\mathcal{O}}$, then

$$
(\omega)_{\ell}\left(\xi_{\ell}^{X}, \xi_{\ell}^{Y}\right)=\left(d p_{d} \wedge d q_{d}\right)_{\ell}\left(\xi_{\ell}^{X}, \xi_{\ell}^{Y}\right)=-\xi_{\ell}^{Y} p_{d} \cdot \xi_{\ell}^{X} q_{d}=\ell[X, Y] .
$$

Finally we address the last condition for adaptable coordinates, and in this case we have that $\mathfrak{g}$ is nilpotent. Set $U_{\epsilon}=O_{\epsilon}, \tau_{d-1}^{\epsilon}(\ell)=\phi_{j_{h}}^{\epsilon}(\ell)$, and $\tau_{d}^{\epsilon}(\ell)=\phi_{j_{k}}^{\epsilon}(\ell)$. From Lemma 3.3 we have that $\tau_{d-1}^{\epsilon}(\ell)$ and $\tau_{d}^{\epsilon}(\ell)$ are $G$-invariant.

Let $\eta^{\circ}$ be a $G_{m}$-invariant function belonging to $\mathcal{E}\left(\Omega^{\circ}\right)$ and define

$$
\eta(\ell)=\eta^{\circ}\left(\exp \left(q_{(1)}(\ell) Z_{1}(\ell)\right) \exp \left(q_{(2)}(\ell) Z_{2}(\ell)\right) \ell\right) .
$$

We claim that $\eta$ is $G$-invariant. Set $X_{1}(\ell)=\tau_{d-1}^{\epsilon}(\ell)^{-1} Z_{1}(\ell)$ and $X_{2}(\ell)=$ $\tau_{d}^{\epsilon}(\ell)^{-1} Z_{2}(\ell)$; if $\ell^{\prime}=\exp \left(t_{1} X_{1}(\ell)\right) \exp \left(t_{2} X_{2}(\ell)\right) s \ell$ where $s \in G_{m}$, then $q_{(1)}\left(\ell^{\prime}\right)=q_{(1)}(\ell)-t_{1} \tau_{d-1}^{\epsilon}(\ell)^{-1}$ and $q_{(2)}\left(\ell^{\prime}\right)=q_{(2)}(\ell)-t_{2} \tau_{d}^{\epsilon}(\ell)^{-1}$, and since $Z_{1}(\ell)$ and $Z_{2}(\ell)$ are $G$-invariant, the claim follows in exactly the same way as Case 1 . For $1 \leq r \leq d-1$ with $k_{r} \notin K_{3}$, put

$$
\tau_{r}^{\epsilon}(\ell)=\tau_{r}^{\epsilon^{\circ}}\left(\exp \left(q_{(1)}(\ell) Z_{1}(\ell)\right) \exp \left(q_{(2)}(\ell) Z_{2}(\ell)\right) \ell\right),
$$

and for each $r$ such that $k_{r} \in K_{3}$, define $\tau_{r}(\ell)$ in the same way. By the claim we have that these functions are also $G$-invariant. The proof that the map $c$ is bijective is similar to the proof in Case 1, and we omit it here. This finishes Case 2.

Case 3: Suppose that $\mathbf{n} \cap \mathbf{e}=\left\{i_{d}, j_{d}\right\}$. Here we have $d^{\circ}=d-1, b^{\circ}=b-1$, and by Lemma 6.1 we have the equality of stabilizers $\mathfrak{s}(\ell)=\mathfrak{s}_{m}(\pi(\ell)), \ell \in \Omega$. Recall that in this case $\mathfrak{s}$ is nilpotent. For simplicity of notation we write $Z=Z_{n-1}$, $\bar{Z}=Z_{n}$.

Consider the complex nilpotent Lie algebra $\mathfrak{t}=\mathfrak{s}_{n-1}$ and let $\pi_{\mathfrak{t}}$ be the restriction mapping $\pi_{\mathfrak{t}}: \mathfrak{s}^{*} \rightarrow \mathfrak{t}^{*}$. For $\ell \in \Omega, \pi_{\mathfrak{t}}(\ell)$ belongs to the fine layer $\Omega^{\mathfrak{t}}$ of all $f \in \mathfrak{t}^{*}$ whose index sequences are $\left(i_{1}, \ldots, i_{d-1}\right),\left(j_{1}, \ldots, j_{d-1}\right)$; in particular $n-1$ is not a jump index for $\pi_{t}(\ell), \ell \in \Omega$. As is well known (see for example, 9, Chapter II, Proposition 1.1]), there is a complex rational function $z(\ell)$ on $\Omega^{\mathrm{t}}$ of the form $z=\psi_{Z}+y$ where $y$ depends only upon $\pi(\ell)$, which is t-invariant on $\Omega^{\mathrm{t}}: \xi_{\ell}^{X} z=0$ for all $\ell \in \Omega^{\mathfrak{t}}$ and $X \in \mathfrak{t}$. We define $q_{d}, p_{d}$ as follows. Again for ease of notation put $Z(\ell)=\rho_{d-1}(Z, \ell)$ and $\bar{Z}(\ell)=\overline{Z(\ell)}$. Set

$$
q_{d}=\frac{z}{\xi_{\ell}^{\bar{Z}(\ell)} z}
$$

and $p_{d}=\bar{z}$. We examine the function $\ell \mapsto \xi_{\ell}^{\bar{Z}(\ell)} z$. It is easily seen that it is s-invariant: indeed for each $X \in \mathfrak{s}$, we have

$$
\xi_{\ell}^{X}\left(\xi^{\bar{Z}(\ell)} z\right)=\xi_{\ell}^{[X, \bar{Z}(\ell)]} z+\xi_{\ell}^{\bar{Z}(\ell)}\left(\xi^{X} z\right),
$$


and since $[X, \bar{Z}(\ell)] \in \mathfrak{s}_{m}$ and $\bar{Z}(\ell)$ belongs to $\mathfrak{s}_{m}^{\ell}$, we have

$$
\xi_{\ell}^{X}\left(\xi^{\bar{Z}(\ell)} z\right)=0
$$

It follows that $q_{d}$ is $\mathfrak{t}$-invariant. Since $\mathfrak{h}(\ell) \subset \mathfrak{t}$ we have $q_{d} \in \mathcal{E}^{0}(\Omega)$. Moreover, we have

$$
\xi_{\ell}^{\bar{Z}} q_{d}=1 .
$$

Now since $\bar{Z}=\bar{Z}(\ell) \bmod \mathfrak{s}_{m}, z$ is $\mathfrak{s}_{m}$-invariant and $\bar{Z}(\ell) \in \mathfrak{s}_{m}^{\ell}$, we have

$$
\xi_{\ell}^{\bar{Z}} z=\xi_{\ell}^{\bar{Z}(\ell)} z=\xi_{\ell}^{\bar{Z}(\ell)} \psi_{Z}=\ell[\bar{Z}(\ell), Z] .
$$

It is easy to show using property (c) for the function $\rho_{d-1}$ that $\ell[\bar{Z}(\ell), Z]$ is pure imaginary. Defining $\tau_{d}(\ell)=-i \xi_{\ell}^{\bar{Z}(\ell)} z$, we see that the relation $p_{d}=i \tau_{d} \overline{q_{d}}$ is satisfied.

Define $q_{k}, p_{k}$ for $k=1, \ldots, d-1$ by $q_{k}=\pi^{*} q_{k}^{\circ}, p_{k}=\pi^{*} p_{k}^{\circ}$, and as in prior cases define

$$
\omega_{d-1}=\sum_{k=1}^{d-1} d p_{k} \wedge d q_{k}, \quad \omega=\sum_{k=1}^{d} d p_{k} \wedge d q_{k} .
$$

Let $X, Y \in \mathfrak{s}$, and fix $\ell \in \Omega$. If $X, Y \in \mathfrak{s}_{m}$, we have

$$
\omega_{\ell}\left(\xi_{\ell}^{X}, \xi_{\ell}^{Y}\right)=\left(\omega_{d-1}\right)_{\ell}\left(\xi_{\ell}^{X}, \xi_{\ell}^{Y}\right)=\ell[X, Y] .
$$

Suppose next that $X=Z(\ell)$, while $Y \in \mathfrak{s}_{m}$. Then $X \in \mathfrak{s}_{m}^{\ell}$, and so for $k=$ $1,2, \ldots, d-1$,

$$
\xi_{\ell}^{X} q_{k}=\xi_{\ell}^{X} p_{k}=0
$$

which says that $\left(\omega_{d-1}\right)_{\ell}\left(\xi_{\ell}^{X}, \xi_{\ell}^{Y}\right)=0$. On the other hand, $q_{d}$ is invariant for $X$ and $Y$. Combining these two observations we find that

$$
\omega_{\ell}\left(\xi_{\ell}^{X}, \xi_{\ell}^{Y}\right)=0=\ell[X, Y] .
$$

Now suppose that $X=\bar{Z}(\ell)$ and $Y \in \mathfrak{t}$. Again $X \in \mathfrak{s}_{m}^{\ell}$, so again $\left(\omega_{d-1}\right)_{\ell}\left(\xi_{\ell}^{X}, \xi_{\ell}^{Y}\right)=$ 0 . Write $Y=c Z+W$, where $W \in \mathfrak{s}_{m}$. Using equation (7.2) and the fact that $\ell[\bar{Z}(\ell), Z]$ is pure imaginary, we obtain

$$
\begin{aligned}
& \omega_{\ell}\left(\xi_{\ell}^{X}, \xi_{\ell}^{Y}\right)=\left(d p_{d} \wedge d q_{d}\right)_{\ell}\left(\xi_{\ell}^{X}, \xi_{\ell}^{Y}\right)=-\xi_{\ell}^{Y} p_{d} \cdot \xi_{\ell}^{X} q_{d} \\
& =-\xi_{\ell}^{c Z+W} p_{d}=-c \xi_{\ell}^{Z} \bar{z}=-\overline{\bar{c} \xi_{\ell}^{\bar{Z}} z} \\
& =-c \overline{\ell[\bar{Z}(\ell), Z]} \\
& =c \ell[\bar{Z}(\ell), Z] \\
& =\ell[\bar{Z}(\ell), c Z+W] \\
& =\ell[X, Y] \text {. }
\end{aligned}
$$

Now in this case $k_{d} \in K_{3}$, and we set $\tau_{r}^{\epsilon}=\pi^{*} \tau_{r}^{\circ \epsilon}$ for $k_{r} \notin K_{3}$. To see that the resulting function $c$ is injective, let $\ell \in U_{\epsilon}$ and $\ell^{\prime} \in \mathcal{O}_{\ell}$. Now $\pi\left(\mathcal{O}_{\ell}\right)=\mathcal{O}_{\pi(\ell)}=$ $G_{m} \pi(\ell)$, so if $\pi(\ell) \neq \pi\left(\ell^{\prime}\right)$, then by induction we have for some $r, p_{r}(\ell)=p_{r}^{\circ}(\pi(\ell)) \neq$ $p_{r}^{\circ}\left(\pi\left(\ell^{\prime}\right)\right)=p_{r}\left(\ell^{\prime}\right)$ (or the same for some $q_{r}$ ). If $\pi(\ell)=\pi\left(\ell^{\prime}\right)$, then since $\ell$ is real it is clear that $\ell(Z) \neq \ell^{\prime}(Z)$, and hence from the form of $p_{d}$, it is clear that $p_{d}(\ell)=p_{d}\left(\ell^{\prime}\right)$ (this obviously also holds for $q_{d}$ in this case). To see that $c$ is surjective, let $e \in E$ and write $e=\left(e^{\circ}, z\right)$, where $z \in \mathbb{C}$. It is clear from the form of $q_{d}$ that we can choose $\ell \in \Omega$ such that $q_{d}(\ell)=z$. Now choosing $u \in G_{m}$ such that $c^{\circ}(u \pi(\ell))=e^{\circ}$, the $G_{m}$-invariance of $q_{d}$ insures that we have $c(u \ell)=e$. This completes the proof. 
We now describe further properties of our system of adaptable coordinates on the layer $\Omega$. Set

$$
\mathcal{V}^{1}(\Omega)=\left\{\xi \in \mathcal{V}(\Omega) \mid\left[\xi, \mathcal{V}^{0}(\Omega)\right] \subset \mathcal{V}^{0}(\Omega)\right\}
$$

and

$$
\mathcal{E}^{1}(\Omega)=\left\{\phi \in \mathcal{E}(\Omega) \mid \xi^{\phi} \in \mathcal{V}^{1}(\Omega)\right\} .
$$

Proposition 7.3. The algebra $\mathcal{E}^{1}(\Omega)$ is the normalizer of $\mathcal{E}^{0}(\Omega)$ in $\mathcal{E}(\Omega)$; that is,

$$
\mathcal{E}^{1}(\Omega)=\left\{\phi \in \mathcal{E}(\Omega) \mid\left\{\phi, \mathcal{E}^{0}(\Omega)\right\} \subset \mathcal{E}^{0}(\Omega)\right\} .
$$

Proof. Let $\phi \in \mathcal{E}^{1}(\Omega)$. Then for any $\eta \in \mathcal{V}^{0}(\Omega)$, we have $\left[\eta, \xi^{\phi}\right] \in \mathcal{V}^{0}(\Omega)$, so

$$
\eta \xi^{\phi}\left(\mathcal{E}^{0}(\Omega)\right)=\left[\eta, \xi^{\phi}\right]\left(\mathcal{E}^{0}(\Omega)\right)+\xi^{\phi} \eta\left(\mathcal{E}^{0}(\Omega)\right)=0 .
$$

Hence $\left\{\phi, \mathcal{E}^{0}(\Omega)\right\}=\xi^{\phi} \mathcal{E}^{0}(\Omega)$ is annihilated by $\mathcal{V}^{0}(\Omega)$. We apply Proposition 7.1 to get $\left\{\phi, \mathcal{E}^{0}(\Omega)\right\} \subset \mathcal{E}^{0}(\Omega)$. On the other hand, let $\phi \in \mathcal{E}(\Omega)$ such that $\left\{\phi, \mathcal{E}^{0}(\Omega)\right\} \subset$ $\mathcal{E}^{0}(\Omega)$, and let $\eta \in \mathcal{V}^{0}(\Omega)$. We have $\gamma=\left[\eta, \xi^{\phi}\right]$ is a vector field with $\gamma_{\ell} \in T_{\ell}\left(\mathcal{O}_{\ell}\right)$ for all $\ell$, so for each $\ell \in \Omega$, we have $Y_{\ell} \in \mathfrak{s}$ such that $\gamma_{\ell}=\ell\left[Y_{\ell}, \cdot\right]$. We must show that $Y_{\ell} \in \mathcal{F}(\ell)$ holds for all $\ell \in \Omega$. Now

$$
0=\eta \xi^{\phi}\left(\mathcal{E}^{0}(\Omega)\right)=\gamma\left(\mathcal{E}^{0}(\Omega)\right)+\xi^{\phi} \eta\left(\mathcal{E}^{0}(\Omega)\right)=\gamma\left(\mathcal{E}^{0}(\Omega)\right)
$$

so that $\gamma$ annihilates $\mathcal{E}^{0}(\Omega)$. Hence for each $\ell \in \Omega$,

$$
0=\gamma_{\ell}(\phi)=d \phi(\ell)\left(\gamma_{\ell}\right)=\ell\left[Y_{\ell}, X_{\ell}^{\phi}\right]
$$

holds for all $\phi \in \mathcal{E}^{0}(\Omega)$, showing that $\left[Y_{\ell}, \mathcal{F}(\ell)\right]=(0)$. Hence $Y_{\ell} \in \mathcal{F}(\ell)$.

Put

$$
c(\ell)=\left(p_{1}(\ell), p_{2}(\ell), \ldots, p_{d}(\ell), q_{1}(\ell), q_{2}(\ell), \ldots, q_{d}(\ell)\right), \ell \in \Omega,
$$

and let $U_{\epsilon}$ be a $G$-invariant covering set. Recall that for $k_{r} \notin K_{3}$, the functions $p_{r}$ and $q_{r}$ can be twisted by $G$-invariant $\mathbb{T}$-valued functions $\tau_{r}^{\epsilon}(\ell), k_{r} \notin K_{3}$, so as to obtain a diffeomorphism of each orbit $\mathcal{O}$ in $\Omega$ with $E=\mathbb{R}^{2 d-2 b} \times \mathbb{C}^{b}$. More precisely, for each $\mathcal{O} \subset \Omega$, let $\{g\}=\mathcal{O} \cap \Sigma, c_{g}=\left.c\right|_{\mathcal{O}}$ and define the subset $V_{g}$ of $\mathbb{C}^{2 d}$ by

$$
V_{g}=\{(p(\ell), q(\ell)) \mid \ell \in \mathcal{O}\} .
$$

Then $V_{g}$ is a real vector subspace of $\mathbb{C}^{2 d}$, and for each $g \in \Sigma$ we have an $\mathbb{R}$ linear isomorphism of real vector spaces $\tau_{g}^{\epsilon}: V_{g} \rightarrow E$ such that $c_{g}^{\epsilon}=\tau_{g}^{\epsilon} \circ c_{g}$ is a diffeomorphism of the orbit $\mathcal{O}=\mathcal{O}_{g}$ with $E$. The map $\tau_{g}^{\epsilon}$ is defined by

$$
\tau_{g}^{\epsilon}\left(p_{1}, \ldots, p_{d}, q_{1}, \ldots, q_{d}\right)=\left(u, v, q_{r_{1}}, \ldots, q_{r_{b}}\right)
$$

where $K_{3}=\left\{k_{r_{1}}, \ldots, k_{r_{b}}\right\}$, and where $u_{r}=\tau_{r}^{\epsilon}(g)^{-1} p_{r}$ and $v_{r}=\tau_{r}^{\epsilon}(g) q_{r}$ when $k_{r} \notin K_{3}$. Write

$$
c_{g}^{\epsilon}(\ell)=\left(u^{\epsilon}(\ell), v^{\epsilon}(\ell), q_{r_{1}}(\ell), \ldots, q_{r_{b}}(\ell)\right), \ell \in \mathcal{O} .
$$

The differential operators $\partial / \partial u_{r}^{\epsilon}, \partial / \partial v_{r}^{\epsilon}$ on $E, k_{r} \notin K_{3}$, define real vector fields $\partial_{u_{r}^{\epsilon}}, \partial_{v_{r}^{\epsilon}}$ in $\mathcal{V}\left(U_{\epsilon}\right)$ : for each $\ell \in U_{\epsilon}$ with $g=\sigma(\ell)$ and $c_{g}^{\epsilon}(\ell)=(u, v, q)$, put

$$
\left(\partial_{u_{r}^{\epsilon}}\right)_{\ell}=d\left(\left(c_{g}^{\epsilon}\right)^{-1}\right)\left(\left.\frac{\partial}{\partial u_{r}}\right|_{(u, v, q)}\right)
$$

and define

$$
\left(\partial_{p_{r}}\right)_{\ell}=\tau_{r}^{\epsilon}(g)^{-1}\left(\partial_{u_{r}^{\epsilon}}\right)_{\ell}
$$


If $U_{\epsilon^{\prime}}$ is any other covering set containing $\ell$, then $\tau_{r}^{\epsilon}(g)= \pm \tau_{r}^{\epsilon^{\prime}}(g)$, and it follows that the definition of $\left(\partial_{p_{r}}\right)_{\ell}$ is independent of the covering set $U_{\epsilon}$. In the same way we define $\partial_{v_{r}^{\epsilon}}$ and we put

$$
\left(\partial_{q_{r}}\right)_{\ell}=\tau_{r}^{\epsilon}(g)\left(\partial_{v_{r}^{\epsilon}}\right)_{\ell}
$$

Thus we obtain vector fields $\partial_{p_{r}}$ and $\partial_{q_{r}}$ on $\Omega$, and since the functions $u_{r}^{\epsilon}, v_{r}^{\epsilon}$ and $\tau_{r}^{\epsilon}$ are analytic on $\Omega$, it is clear that $\partial_{p_{r}}$ and $\partial_{q_{r}}$ are smooth. Observe that for each $\ell \in \Omega$ with $g=\sigma(\ell), p_{r} \circ\left(c_{g}^{\epsilon}\right)^{-1}=\tau_{r}^{\epsilon}(g) u_{r}$ and $q_{r} \circ\left(c_{g}^{\epsilon}\right)^{-1}=\tau_{r}^{\epsilon}(g)^{-1} v_{r}$, so $\partial_{p_{r}}\left(p_{r}\right)=\partial_{q_{r}}\left(q_{r}\right)=1$.

Suppose now that $k_{r} \in K_{3}$. Then the definition of $c_{g}^{\epsilon}$ in the $q_{r}$ direction is the same for all covering sets $U_{\epsilon}$, so with $x_{r}=\mathfrak{R e} q_{r}$ and $y_{r}=\mathfrak{I m} q_{r}$, we define

$$
\left(\partial_{x_{r}}\right)_{\ell}=d\left(\left(c_{g}^{\epsilon}\right)^{-1}\right)\left(\left.\frac{\partial}{\partial x_{r}}\right|_{(u, v, q)}\right) \text {, }
$$

and the definition is independent of the covering set $U_{\epsilon}$. Similarly we define $\left(\partial_{y_{r}}\right)_{\ell}$, and so when $k_{r} \in K_{3}$ we have the vector field $\partial_{q_{r}} \in \mathcal{V}(\Omega)$ defined by

$$
\partial_{q_{r}}=\frac{1}{2}\left(\frac{\partial}{\partial x_{r}}-i \frac{\partial}{\partial y_{r}}\right) \text {. }
$$

Let $\tau_{r}=\tau_{r}(\ell)$ be the real-valued $G$-invariant function such that $p_{r}(\ell)=i \tau_{r}(\ell) \bar{q}_{r}(\ell)$. For each $\ell \in \Omega$ with $g=\sigma(\ell)$, set

$$
\left(\partial_{p_{r}}\right)_{\ell}=\frac{1}{i \tau_{r}(g)}\left(\bar{\partial}_{q_{r}}\right)_{\ell}=\frac{1}{2 i \tau_{r}(g)}\left(\frac{\partial}{\partial x_{r}}+i \frac{\partial}{\partial y_{r}}\right)_{\ell} .
$$

Then $\partial_{p_{r}}$ also belongs to $\mathcal{V}(\Omega)$, and it is clear that $\partial_{q_{r}}\left(q_{r}\right)=\partial_{p_{r}}\left(p_{r}\right)=1$.

Now it is immediate from the definitions that for all $1 \leq r, r^{\prime} \leq d, \partial_{q_{r}}\left(p_{r^{\prime}}\right)=$ $\partial_{p_{r}}\left(q_{r^{\prime}}\right)=0$, and for $r^{\prime} \neq r, \partial_{p_{r}}\left(p_{r^{\prime}}\right)=\partial_{q_{r}}\left(q_{r^{\prime}}\right)=0$ as well. Hence for each $\ell \in \mathcal{O}$, the $2 d$ tangent vectors $\left(\partial_{p_{r}}\right)_{\ell},\left(\partial_{q_{r}}\right)_{\ell}, 1 \leq r \leq d$, are independent, and we have

$$
T_{\ell}(\mathcal{O})_{\mathbb{C}}=\mathbb{C}-\operatorname{span}\left(\left\{\left(\partial_{p_{r}}\right)_{\ell},\left(\partial_{q_{r}}\right)_{\ell} \mid 1 \leq r \leq d\right\}\right) .
$$

For any $\phi \in \mathcal{E}(\Omega)$, write the associated Hamiltonian vector field $\xi^{\phi} \in \mathcal{V}(\Omega)$ as $\xi^{\phi}=\sum a_{r} \partial_{p_{r}}+b_{r} \partial_{q_{r}}$. For each orbit $\mathcal{O}=\mathcal{O}_{g} \subset \Omega$, let $\omega_{g}$ be the canonical non-degenerate two-form on $\mathcal{O}$. Recall that $\xi^{\phi}$ is defined on $\mathcal{O}$ by the relation

$$
\left(\omega_{g}\right)_{\ell}\left(\xi_{\ell}^{\phi}, \cdot\right)=-d \phi_{\ell}
$$

Now by Theorem 7.2, the two-form $\omega=\sum_{r} d p_{r} \wedge d q_{r}$ restricts to $\omega_{g}$ on $\mathcal{O}_{g}$. With our notation, it follows that

$$
b_{r}=\partial_{p_{r}}(\phi), \quad a_{r}=-\partial_{q_{r}}(\phi) .
$$

Hence

$$
\xi^{\phi}=\sum_{r=1}^{d} \partial_{p_{r}}(\phi) \partial_{q_{r}}-\partial_{q_{r}}(\phi) \partial_{p_{r}},
$$

and the Poisson bracket for functions $\phi, \psi \in \mathcal{E}(\Omega)$ is

$$
\{\phi, \psi\}=\xi^{\phi}(\psi)=\sum_{r=1}^{d} \partial_{p_{r}}(\phi) \partial_{q_{r}}(\psi)-\partial_{q_{r}}(\phi) \partial_{p_{r}}(\psi) .
$$

As a word of caution, we note that although $\phi$ and $\psi$ are understood to be defined on an open set $U$ containing the layer $\Omega$, the preceding identity holds only on $\Omega$, 
and not necessarily on the entire open set $U$. Now from Theorem 7.2 we have that $\partial_{p_{r}}=-\xi^{q_{r}} \in \mathcal{V}^{0}(\Omega)$ holds for each $1 \leq r \leq d$, and by comparing dimensions we see that for each $\ell \in \Omega$,

$$
\mathbb{C}-\operatorname{span}\left\{\left(\partial_{p_{r}}\right)_{\ell} \mid 1 \leq r \leq d\right\}=\mathcal{F}(\ell) .
$$

We use these objects to give a description of the algebras $\mathcal{E}^{0}(\Omega)$ and $\mathcal{E}^{1}(\Omega)$. Let $\phi$ be a smooth function on $\Omega$. First, we have $\phi \in \mathcal{E}^{0}(\Omega)$ if and only if $\partial_{p_{r}}(\phi)=$ $0,1 \leq r \leq d$ holds. Thus $\phi \in \mathcal{E}^{0}(\Omega)$ if and only if for each $g \in \Sigma, \phi \circ\left(c_{g}^{\epsilon}\right)^{-1}$ satisfies

$$
\phi \circ\left(c_{g}^{\epsilon}\right)^{-1}(u, v, q)=\phi \circ\left(c_{g}^{\epsilon}\right)^{-1}(0, v, q)
$$

for all $u$, and $\phi \circ\left(c_{g}^{\epsilon}\right)^{-1}$ is holomorphic in $q_{r_{1}}, \ldots, q_{r_{b}}$. Put $V_{g}^{0}=\left\{\left(q_{1}(\ell), \ldots, q_{d}(\ell)\right) \mid\right.$ $\left.\ell \in \mathcal{O}_{g}\right\}$ and $V_{g}^{1}=\left\{\left(p_{1}(\ell), \ldots, p_{d}(\ell)\right) \mid \ell \in \mathcal{O}_{g}\right\}$, and regard $V_{g}^{0}$ and $V_{g}^{1}$ as subspaces of $V_{g}$. Then the preceding shows that $\phi$ belongs to $\mathcal{E}^{0}(\Omega)$ if and only if $\phi \circ c_{g}^{-1}$ depends only upon $q_{1}, \ldots, q_{d}$; that is,

$$
\left(\phi \circ c_{g}^{-1}\right)(p, q)=\left(\phi \circ c_{g}^{-1}\right)(0, q), \quad p \in V_{g}^{1},
$$

holds for each $g$. In this case we may write $\left(\phi \circ c_{g}^{-1}\right)(q)$.

Next, we recall that $\phi$ belongs to $\mathcal{E}^{1}(\Omega)$ if and only if $\left[\xi^{\phi}, \mathcal{V}^{0}(\Omega)\right] \subset \mathcal{V}^{0}(\Omega)$. A simple computation using formula (7.3) shows that this condition is equivalent to

$$
\partial_{p_{r^{\prime}}} \partial_{p_{r}}(\phi)=0,1 \leq r, r^{\prime} \leq d .
$$

If $\phi$ has the form $\phi=\sum_{r=1}^{d} a_{r} p_{r}+a_{0}$ where $a_{r} \in \mathcal{E}^{0}(\Omega), 0 \leq r \leq d$, then clearly $\phi \in \mathcal{E}^{1}(\Omega)$. On the other hand, if $\phi \in \mathcal{E}^{1}(\Omega)$, then for each $\ell \in \Omega, g=\sigma(\ell)$, choose a covering set $U_{\epsilon}$ containing $\ell$. Then we have

$$
\frac{\partial^{2}}{\partial u_{r}^{\prime} \partial u_{r}}\left(\phi \circ\left(c_{g}^{\epsilon}\right)^{-1}\right)=0
$$

for all $r, r^{\prime}$, so

$$
\phi \circ\left(c_{g}^{\epsilon}\right)^{-1}(u, v, q)=\sum_{k_{r} \notin K_{3}} \alpha_{r}^{\epsilon}(v, q, g) u_{r}+\sum_{k_{r} \in K_{3}} \alpha_{r}^{\epsilon}(v, q, g) \bar{q}_{r}+\alpha_{0}^{\epsilon}(v, q, g),
$$

where $\alpha_{r}^{\epsilon}$ is holomorphic in $q=q_{r_{1}}, \ldots, q_{r_{b}}$. Now for each $r$ such that $k_{r} \notin K_{3}$ (as well as $r=0)$, set $a_{r}^{\epsilon}=\alpha_{r}^{\epsilon}(\cdot, \cdot, g) \circ c_{g}^{\epsilon}$, and if $k_{r} \in K_{3}$, put $a_{r}^{\epsilon}=\left(i \tau_{r}(g)\right)^{-1} \alpha_{r}(\cdot, \cdot, g) \circ$ $c_{g}^{\epsilon}$. Then the $a_{r}^{\epsilon}$ are smooth functions and we have

$$
\left.\phi\right|_{U_{\epsilon}}=\sum_{r=1}^{d} a_{r}^{\epsilon} p_{r}+a_{0}^{\epsilon} .
$$

Now since $U_{\epsilon} \cap U_{\epsilon^{\prime}}$ is dense in $\Omega$, the preceding expression is independent of the choice of covering set and the functions $a_{r}$ belong to $\mathcal{E}^{0}(\Omega)$. Thus we have shown that

$$
\mathcal{E}^{1}(\Omega)=\left\{\sum_{r=1}^{d} a_{r} p_{r}+a_{0} \mid a_{r} \in \mathcal{E}^{0}(\Omega)\right\} .
$$

With these notation and remarks, we have the following.

Corollary 7.4. Let $\mathfrak{g}$ be an exponential Lie algebra, and $\left(Z_{1}, \ldots, Z_{n}\right)$ an adaptable basis for $\mathfrak{s}=\mathfrak{g}^{\mathbb{C}}$. Let $\Omega=\Omega_{\mathbf{e}, \mathbf{j}, \varphi}$ be an ultrafine layer and $\left(p_{1}, \ldots, p_{d}, q_{1}, \ldots, q_{d}\right)$ the adaptable coordinates built in Theorem 7.2. We have the following:

(a) The Vergne geometrical polarization is given by

$$
\mathcal{F}(\ell)=\mathbb{C}-\operatorname{span}\left\{\left(\partial_{p_{r}}\right)_{\ell} \mid 1 \leq r \leq d\right\} .
$$


(b) The algebra $\mathcal{E}^{0}(\Omega)$ consists of all smooth functions $\phi$ such that for each orbit $\mathcal{O}=\mathcal{O}_{g}, \phi \circ c_{g}^{-1}$ depends only upon $q_{1}, \ldots, q_{d}$, and the algebra $\mathcal{E}^{1}(\Omega)$ consists of all smooth functions $\phi$ of the form

$$
\phi=\sum_{r} a_{r} p_{r}+a_{0}
$$

where $a_{r} \in \mathcal{E}^{0}(\Omega), 0 \leq r \leq d$.

(c) For each $X \in \mathfrak{g}$, the function $\psi_{X}$ belongs to $\mathcal{E}^{1}(\Omega)$.

Proof. Parts (a) and (b) are demonstrated in the remarks preceding the corollary.

For part (c), let $X \in \mathfrak{g}$; we show that $\partial_{p_{r}}\left(\psi_{X}\right)$ belongs to $\mathcal{E}^{0}(\Omega)$.

For each orbit $\mathcal{O}=\mathcal{O}_{g}$ we have smooth functions $F: V_{g} \times \mathbb{R} \rightarrow V_{g}^{0}$ and $G$ : $V_{g} \times \mathbb{R} \rightarrow V_{g}^{1}$ such that

$$
\left(c_{g} \circ \operatorname{Ad}^{*}(\exp (t X)) \circ c_{g}^{-1}\right)(p, q)=(F(p, q, t), G(p, q, t)) .
$$

For each $t \in \mathbb{R}$, we put $q_{r}^{t}=q_{r}(\exp (t X) \cdot)$; then we can write

$$
\left(q_{r}^{t} \circ c_{g}^{-1}\right)(p, q)=\left(q_{r} \circ c_{g}^{-1}\right)(F(p, q, t), G(p, q, t))=G_{r}(p, q, t) .
$$

Now for each $\ell \in \Omega$, we have $\exp (t X)^{*} \mathcal{F}(\ell)=\mathcal{F}\left(\ell^{\prime}\right)$, and it follows that $q_{r}^{t} \in \mathcal{E}^{0}(\Omega)$ holds for each $t$. Hence $G_{r}$ depends only upon $q$ and $t$. Taking the derivative with respect to $t$, we see that

$$
\partial_{p_{r}}\left(\psi_{X}\right)=\xi^{q_{r}}\left(\psi_{X}\right)=-\xi^{X}\left(q_{r}\right)=\left.\frac{\partial}{\partial t}\right|_{t=0} G_{r}(q, t)
$$

belongs to $\mathcal{E}^{0}(\Omega)$.

We conclude this paper with an illustration of the preceding constructions as applied to our three examples.

Example 7.1. As in Examples 1.1, 2.1] and 4.1, let

$$
\mathfrak{g}=\operatorname{span}\left\{Z_{1}, Z_{2}, Y, X_{1}, X_{2}, A\right\},
$$

where $\left[X_{j}, Y\right]=Z_{j},\left[A, Z_{1}+i Z_{2}\right]=(1+i)\left(Z_{1}+i Z_{2}\right)$, and $\left[A, X_{1}+i X_{2}\right]=(1+i)$ $\left(X_{1}+i X_{2}\right)$. Choose the adaptable basis $\left(Z_{1}+i Z_{2}, Z_{1}-i Z_{2}, Y, X_{1}+i X_{2}, X_{1}-i X_{2}, A\right)$ of $\mathfrak{s}$. The basis coordinates for $\ell \in \mathfrak{g}^{*}$ are $\ell=(z, \bar{z}, y, x, \bar{x}, a)$. Recall there are two ultrafine layers that have non-trivial orbits.

1. $\Omega=\Omega_{\mathbf{e}, \mathbf{j}}$, where $\mathbf{e}=\{1,3,4,6\}, \mathbf{j}=(6,4), \varphi=\{1\}$. Beginning with $\mathfrak{s}_{5}=$ $\mathbb{C}-\operatorname{span}\left\{Z_{1}+i Z_{2}, Z_{1}-i Z_{2}, Y, X_{1}+i X_{2}, X_{1}-i X_{2}\right\}$, see that $\pi_{5}(\Omega) \subset \Omega_{\mathbf{e}^{1} \mathbf{j}^{1}}=\Omega_{\mathrm{e}^{1}}$ where $\mathbf{e}^{1}=\{3,4\}$. Applying the first $q$-function formula of Proposition 6.3, we obtain the adaptable coordinates

$$
q_{1}^{1}=\frac{y}{|z|^{2}}, \quad p_{1}^{1}=\mathfrak{R e}(\bar{z} x)
$$

for $\Omega_{\mathbf{e}^{1}}$. Now we go up to $\mathfrak{s}$. Applying the second $q$-function formula of Proposition 6.3. we obtain $Z_{6}(\ell)=A$,

$$
q_{2}=q_{2}^{2}=\ln |z|, \quad p_{2}=p_{2}^{2}=a .
$$

Set $\ell^{\prime}=\exp \left(q_{2}(\ell) A \ell\right)$, with $\ell^{\prime}=\left(z^{\prime}, \overline{z^{\prime}}, y^{\prime}, x^{\prime}, \overline{x^{\prime}}, a^{\prime}\right)$. Then

$$
z^{\prime}=\frac{z}{|z|^{1+i}}, \quad y^{\prime}=y, \quad x^{\prime}=\frac{x}{|z|^{1+i}}
$$


so from the proof of Theorem 7.2 . Case 1, we get

$$
q_{1}=q_{1}^{2}=y, \quad p_{1}=p_{1}^{2}=\mathfrak{R e}\left(\overline{z^{\prime}} x^{\prime}\right)=\mathfrak{R e}\left(\frac{x}{z}\right) .
$$

We can write coordinates for $\ell \in \Omega$ using the notation of Example 4.1]

$$
\ell=\left(e^{q_{2}(1+i)} e^{i \theta}, e^{q_{2}(1-i)} e^{-i \theta}, q_{1},\left(p_{1}+i \xi\right) e^{q_{2}(1+i)} e^{i \theta},\left(p_{1}-i \xi\right) e^{q_{2}(1-i)} e^{-i \theta}, p_{2}\right) .
$$

2. $\Omega=\Omega_{\mathbf{e}, \mathbf{j}}$, where $\mathbf{e}=\{4,6\}, \mathbf{j}=(6), \varphi=\{4\}$. In this case the layer in $\mathfrak{s}_{5}$ which contains $\pi_{5}(\Omega)$ has trivial orbits. Hence we directly obtain

$$
q_{1}=\ln |x|, \quad p_{1}=a,
$$

and the coordinates for $\ell$ are, with the notation of 4.1 ,

$$
\ell=\left(0,0, \eta, e^{q_{1}(1+i)} e^{i \theta}, e^{q_{1}(1-i)} e^{-i \theta}, p_{1}\right) .
$$

Example 7.2. As in Examples 1.2, 2.2 and 4.2, $\mathfrak{g}$ is the Lie algebra for which $\mathfrak{s}=\mathfrak{g}_{\mathbb{C}}$ has the adaptable basis $\{Z, \bar{Z}, Y, \bar{Y}, X, \bar{X}, A\}$ and relations

$$
\begin{aligned}
& {[X, Y]=0,[X, \bar{Y}]=(1-i) \bar{Z},} \\
& {[A, X]=(1+i) X,[A, Y]=(1+2 i) Y,[A, Z]=(2+i) Z .}
\end{aligned}
$$

Again we use the basis coordinates $\ell=(z, \bar{z}, y, \bar{y}, x, \bar{x}, a)$. Here there are three ultrafine layers with non-trivial orbits.

1. $\Omega=\Omega_{\mathbf{e}, \mathbf{j}}$ with $\mathbf{e}=\{1,3,4,5,6,7\}, \mathbf{j}=(7,6,5), \varphi=\{1\}$. The layer in $\mathfrak{s}_{6}^{*}$ containing $\pi_{6}(\Omega)$ is $\Omega_{\mathbf{e}^{1}, \mathbf{j}^{1}}$, where $\mathbf{e}^{1}=\{3,4,5,6\}$ and $\mathbf{j}^{1}=(6,5)$. Applying the $q$-function formula of Proposition 6.4 we obtain the adaptable coordinates for $\Omega_{\mathbf{e}^{1}, \mathbf{j}^{1}}$ :

$$
\begin{array}{ll}
q_{1}^{1}=\frac{\mathfrak{R e}(y)}{5|z|^{2}}, & p_{1}^{1}=\mathfrak{R e}\left(\frac{(1+i) z x}{2}\right), \\
q_{2}^{1}=\frac{\mathfrak{I m}(y)}{5|z|^{2}}, & p_{2}^{1}=\mathfrak{I m}\left(\frac{(1+i) z x}{2}\right) .
\end{array}
$$

Going up from $\mathfrak{s}_{6}$ to $\mathfrak{s}$, we use the second $q$-function formula of Proposition 6.3 to define $q_{3}$ :

$$
q_{3}=\frac{1}{2} \ln |z|, \quad p_{3}=a,
$$

and with $\ell^{\prime}=\exp \left(q_{3}(\ell) A \ell\right)=\left(z^{\prime}, \overline{z^{\prime}}, y^{\prime}, \overline{y^{\prime}}, x^{\prime}, \overline{x^{\prime}}, a^{\prime}\right)$ we get

$$
z^{\prime}=|z|^{-(2+i) / 2} z, \quad y^{\prime}=|z|^{-(1+2 i) / 2} y, \quad x^{\prime}=|z|^{-(1+i) / 2} x .
$$

Applying the proof of Theorem 17.2, Case 2, we find that the real coordinates $q_{1}, q_{2}, p_{1}$ and $p_{2}$ are given by

$$
q_{1}+i q_{2}=\frac{1}{5}|z|^{-(1+2 i) / 2} y
$$

and

$$
p_{1}+i p_{2}=\frac{1+i}{2}|z|^{-(3+2 i) / 2} z x .
$$

Thus the coordinates for $\ell$ can be written as

$$
z=e^{(2+i) q_{3}} e^{i \theta}, y=5 e^{q_{3}(1+2 i)}\left(q_{1}+i q_{2}\right), x=\frac{2}{1+i} e^{q_{3}(1+i)} e^{-i \theta}, a=p_{3} .
$$

2. $\Omega=\Omega_{\mathbf{e}, \mathbf{j}}$, where $\mathbf{e}=\{3,7\}, \mathbf{j}=(7), \varphi=\{3\}$. There is only one step, since the $\pi_{6}(\Omega)$ consists of trivial orbits. We have

$$
q=\ln |y|, \quad p=a,
$$


so that $\ell$ is just

$$
\ell=\left(0,0, e^{q(1+2 i)} e^{i \theta}, e^{q(1-2 i)} e^{-i \theta}, e^{q(1+i)} \xi, e^{q(1-i) \bar{\xi}}, p\right) .
$$

3. $\mathbf{e}=\{5,7\}, \mathbf{j}=(5), \varphi=\{5\}$. Again there is only one step:

$$
q=\ln |x|, \quad p=a,
$$

and $\ell$ is just

$$
\ell=\left(0,0,0,0, e^{q(1+i)} e^{i \theta}, e^{q(1-i)} e^{-i \theta}, p\right) .
$$

Example 7.3. As in Examples 1.3, 2.3 and 4.3, let $\mathfrak{g}$ be the Lie algebra for which $\mathfrak{s}$ has the adaptable basis $\{Z, X+i Y, X-i Y, A\}$ and relations

$$
[X, Y]=Z,[A, X+i Y]=(1+i)(X+i Y),[A, Z]=2 Z \text {. }
$$

Write $\ell=(z, x+i y, x-i y, a)$.

1. $\Omega=\Omega_{\mathbf{e}, \mathbf{j}}$, where $\mathbf{e}=\{1,2,3,4\}, \mathbf{j}=(3,4), \varphi=\{1\}$. Note that $2 \in K_{3}$ here: $Z_{i_{2}}=X+i Y$ and $Z_{j_{2}}=X-i Y$. Now $\pi_{3}(\Omega)$ coincides with the layer $\Omega_{\mathbf{e}^{1}}$ where $\mathbf{e}^{1}=\{2,3\}=\left\{i_{1}^{3}, j_{1}^{3}\right\}$ and $1 \in K_{3}^{3}$, so that we apply Case 3 of Theorem 7.2 to obtain

$$
q_{1}^{1}=\frac{x+i y}{2 i z}, \quad p_{1}^{1}=x-i y .
$$

Going up to $\mathfrak{s}$ in the same way as the preceding examples, we have

$$
\begin{aligned}
& q_{2}=\ln |z|, \quad p_{2}=a, \\
& q_{1}=q_{1}^{2}=\frac{1}{2 i} \operatorname{sign}(z)|z|^{-i}(x+i y), \quad p_{1}=p_{1}^{2}=|z|^{-(1-i)}(x-i y) .
\end{aligned}
$$

If $\epsilon=\operatorname{sign}(z)$ so that $g=\{(\epsilon, 0,0,0) \mid \epsilon= \pm 1\}$, then the coordinates for $\ell$ are

$$
\ell=\left(\epsilon e^{q_{2}}, 2 i \epsilon e^{i q_{2}} q_{1}, e^{q_{2}(1-i)} p_{1}, p_{2}\right) .
$$

2. $\mathbf{e}=\{2,4\}, \mathbf{j}=(4), \varphi=\{2\}$. We directly obtain the coordinates

$$
q=\ln |x+i y|, \quad p=a,
$$

and so

$$
\ell=\left(0, e^{q(1+i)} e^{i \theta}, e^{q(1-i)} e^{-i \theta}, p\right)
$$

\section{REFERENCES}

1. Arnal, D., Ben Ammar, M., Currey, B., and Dali, B., Canonical coordinates for coadjoint orbits of completely solvable Lie groups, Jour. Lie Theory No. 2 (2005) 521-560.

2. Arnal, D., And Cortet, J. C. Representations * des groupes exponentiels, Jour. Funct. Anal. 92 (1990), 103-135. MR.1064689 (91h:22014)

3. Arnal, D., And Dali, B. Déformations polarisées d'algèbres sur les orbites coadjointes d'un groupe exponentiel, Annales de la Faculté des Sciences de Toulouse, Vol. IX, no. 1, (2000), 31-54. MR1815939 (2002b:22014)

4. Currey, B. Explicit orbital parameters and the Plancherel measure for exponential Lie groups, Pac. Jour. Math. 219, No. 1 (2005), 101-142. MR2174222 (2006i:22006)

5. Currey, B. The structure of the space of coadjoint orbits of an exponential solvable Lie group, Trans. Amer. Math. Soc. 332 (1992), 241-269. MR.1046014 (92j:22020)

6. Pedersen, N. V., On the symplectic structure of coadjoint orbits of (solvable) Lie groups and applications, Math. Ann. 281 (1988), 633-669. MR958263 (90c:22024)

7. Pedersen, N. V., Geometric quantization and the universal enveloping algebra of a nilpotent Lie group, Trans. Amer. Math. Soc. 315, No. 2 (1989), 511-563. MR967317 (90c:22026) 
8. Pukanszky, L., On the unitary representations of exponential Lie groups, J. Funct. Anal. 2 (1968), 73-113. MR0228625 (37:4205)

9. Pukanszky, L., Unitary representations of solvable Lie groups, Ann. Sci. Ec. Norm. Sup. 4 (1971), 457-608. MR0439985 (55:12866)

Institut de Mathématiques de Bourgogne, Université de Bourgogne, CNRS UMR 5584, BP47870, F-21078 Dijon Cedex, France

E-mail address: didier.arnal@u-bourgogne.fr

Department of Mathematics and Computer Science, St. Louis University, St. Louis, Missouri 63103

E-mail address: curreybn@slu.edu

Département de Mathématiques, Faculté des Sciences de Bizerte, 7021 Zarzouna, Bizerte, Tunisie

E-mail address: bechir.dali@fss.rnu.tn 